\title{
Flag algebras and tournaments
}

\author{
Leonardo Nagami Coregliano
}

Dissertation PRESENTED

$\mathrm{TO}$

Instituto De Matemática e Estatística

$\mathrm{OF}$

Universidade of SÃo Paulo

FOR

OBTAINMENT OF THE TITLE

$\mathrm{OF}$

MASTER of ScIEnCES

Program: Computer Science

Adviser: Prof. Dr. Yoshiharu Kohayakawa

During the development of this work the author received funding from Fundação de Amparo à Pesquisa do Estado de São Paulo (FAPESP), grants no. 2013/23720-9 and 2014/15134-5, and Coordenação de Aperfeiçoamento de Pessoal de Nível Superior (CAPES).

This research was partially done while the author was visiting The University of Chicago.

The author acknowledges the support of CEPID/FAPESP NeuroMat Project, Proc. 2013/07699-0 and of the FAPESP/Thematic Project Proc. 2013/03447-6. 



\section{Flag algebras and tournaments}

This version of the dissertation contains the corrections and changes suggested by the Examination Committee during the defense of the original version of the work, done on $05 / 08 / 2015$. A copy of the original version is available in Instituto de Matemática e Estatística of Universidade of São Paulo.

Examination Committee:

- Prof. Dr. Yoshiharu Kohayakawa (president) - IME-USP

- Prof. Dr. Jie Han - IME-USP

- Prof. Dr. Carlos Hoppen - UFRGS 



\title{
Acknowledgements
}

\author{
I am grateful
}

to Prof. Yoshiharu Kohayakawa

for introducing me to the field of asymptotic combinatorics; and for advising me very wisely;

to Prof. Alexander Razborov

for the hospitality while I was visiting The University of Chicago;

to Irroko Nagami

for all the love and support;

to Valdemir Aparecido Coregliano

for all the love and support;

to Stephany Mayumi Araki

for making my life even happier. 


\section{Abstract}

COREGLIANO, Leonardo N. Flag algebras and tournaments. Dissertation - Instituto de Matemática e Estatística, Universidade of São Paulo, São Paulo, 2015. Alexander A. Razborov (2007) developed the theory of flag algebras to compute the minimum asymptotic density of triangles in a graph as a function of its edge density. The theory of flag algebras, however, can be used to study the asymptotic density of several combinatorial objects.

In this dissertation, we present two original results obtained in the theory of tournaments through application of flag algebra proof techniques.

The first result concerns minimization of the asymptotic density of transitive tournaments in a sequence of tournaments, which we prove to occur if and only if the sequence is quasirandom. As a byproduct, we also obtain new quasi-random characterizations and several other flag algebra elements whose density is minimized if and only if the sequence is quasi-random.

The second result concerns a class of equivalent properties of a sequence of tournaments that we call quasi-carousel properties and that, in a similar fashion as quasi-random properties, force the sequence to converge to a specific limit homomorphism. Several quasi-carousel properties, when compared to quasi-random properties, suggest that quasi-random sequences and quasi-carousel sequences are the furthest possible from each other within the class of almost balanced sequences.

Keywords: Asymptotic combinatorics, flag algebra, tournaments, quasi-random, extremal problems. 


\section{Resumo}

COREGLIANO, Leonardo N. Álgebras de flags e torneios. Dissertação - Instituto de Matemática e Estatística, Universidade de São Paulo, São Paulo, 2015. Alexander A. Razborov (2007) desenvolveu a teoria de álgebras de flags para calcular a densidade assintótica mínima de triângulos em um grafo em função de sua densidade de arestas. A teoria das álgebras de flags, contudo, pode ser usada para estudar densidades assintóticas de diversos objetos combinatórios.

Nesta dissertação, apresentamos dois resultados originais obtidos na teoria de torneios através de técnicas de demonstração de álgebras de flags.

O primeiro resultado compreende a minimização da densidade assintótica de torneios transitivos em uma sequência de torneios, a qual provamos ocorrer se e somente se a sequência é quase aleatória. Como subprodutos, obtemos também novas caracterizações de quase aleatoriedade e diversos outros elementos da álgebra de flags cuja densidade é minimizada se e somente se a sequência é quase aleatória.

O segundo resultado compreende uma classe de propriedades equivalentes sobre uma sequência de torneios que chamamos de propriedades quase carrossel e que, de uma forma similar às propriedades quase aleatórias, forçam que a sequência convirja para um homomorfismo limite específico. Várias propriedades quase carrossel, quando comparadas às propriedades quase aleatórias, sugerem que sequências quase aleatórias e sequências quase carrossel estão o mais distantes possível umas das outras na classe de sequências quase balanceadas.

Palavras-chave: Combinatória assintótica, álgebras de flags, torneios, quase aleatório, problemas extremais. 


\section{Contents}

List of symbols $\quad$ ix

List of figures $\quad$ XV

List of tables $\quad$ xvii

$\begin{array}{ll}\text { Introduction } & 1\end{array}$

1 Flag algebras $\quad 5$

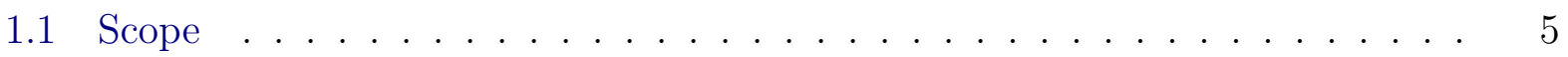

1.2 Basic definitions . . . . . . . . . . . . . . . . . . . 8

1.3 Homomorphism extensions . . . . . . . . . . . . . . . . . . . . . . 15

1.4 Interpretation homomorphisms . . . . . . . . . . . . . . . . . . . 19

1.4.1 Algebras of constants: upward operator . . . . . . . . . . . . . 20

1.4.2 Inductive arguments . . . . . . . . . . . . . . . . 22

1.4.3 "Genuine" interpretations . . . . . . . . . . . . . . . . . . . . 23

2 Minimization of transitive tournaments and quasi-randomness 25

2.1 An introduction to tournament quasi-randomness . . . . . . . . . . . . 25

2.2 Minimization of transitive tournaments . . . . . . . . . . . . . . . . . . 28

2.3 Other quasi-random properties . . . . . . . . . . . . . . . . 30

2.4 Further extremal quasi-randomness properties . . . . . . . . . . . . 33

3 Carousel tournaments and quasi-carouselness 41

3.1 Locally transitive tournaments . . . . . . . . . . . . . . . . . 41

3.2 The carousel homomorphism and the quasi-carousel properties . . . . . . . . 42

3.3 Quasi-carouselness proofs . . . . . . . . . . . . . . . . . . 45

4 Final remarks $\quad 51$

$\begin{array}{ll}\text { A First-order logic } & 57\end{array}$

$\begin{array}{ll}\text { B Other topics in flag algebra } & \mathbf{6 1}\end{array}$

B.1 Semidefinite method . . . . . . . . . . . . . . . . 61 
B.2 Differential method . . . . . . . . . . . . . . . . . . . . . 64 $\begin{array}{ll}\text { Bibliography } & 67\end{array}$

$\begin{array}{ll}\text { Index } & 71\end{array}$ 


\title{
List of symbols
}

\author{
$(U, I): T_{1} \rightsquigarrow T_{2}$ \\ Open interpretation $(U, I)$ of $T_{1}$ in $T_{2}, 19,24,59$ \\ $(n)_{k}=n(n-1) \cdots(n-k+1)$ \\ Decreasing factorial, 17 \\ $<_{\tau}$ \\ Order induced by the permutation $\tau, 7,8$
}

$\llbracket \cdot \rrbracket_{\sigma, \eta} \quad$ Downward operator relative to $\sigma$ and $\eta, 16-18,20,21,23,28-31,35-37,62-64,66$ $[k]=\{1,2, \ldots, k\} \quad$ Set of positive integers smaller or equal to $k, 7-9,14,16,17,19-22,25$, $42,46,47,53,58$

$\alpha$ 1-flag on $T_{\text {Tournament }}$ where the labelled vertex beats the unlabelled vertex, 28, 31, 34-38, $55,63,64$

$\beta \quad$ 1-flag on $T_{\text {Tournament }}$ where the labelled vertex is beaten by the unlabelled vertex, 28 , $34-36,38,55,63,64$

$\cong$

$|F|$

$\delta(A)$

$\mu^{1}$

$\nabla f$

$\partial_{1}$

$\phi_{\text {Graph,qr }}$

$\phi_{\text {Perm,qr }}$

$\phi_{\mathrm{R}}$

$\phi_{\operatorname{Tr}}$

$\phi^{\sigma, \eta}$ 36-38, 42-46, 48, 55, 63, 64, 66

$\phi_{\mathrm{qr}}$

Quasi-random homomorphism, 25, 26, 28-34, 36-38, 41, 43, 44, 52, 55
Isomorphic, 9, 15, 19, 20, 22, 65

Size of the flag $F, 6,7,9,13-17,19-21,31,45,48,49,62$

Boundary of the set $A, 18$

Operator $\mu^{1}, 65$

Gradient of function $f, 65$

Vertex deletion differential operator, 65, 66

Quasi-random homomorphism in $T_{\text {Graph }}, 51$ Quasi-random homomorphism in $T_{\text {Perm }}, 51,52$

Carousel homomorphism, 41-46, 48, 53

Transitive homomorphism, 29, 32, 33
Extension of homomorphism $\phi$ to type $\sigma$ through $\eta, 15,16,18,20,21,23,27-33$, 
$\pi^{(U, I)}$ Interpretation homomorphism relative to the open interpretation $(U, I), 19,20,22$, 24,62

$\pi^{F, \eta} \quad$ Interpretation homomorphism relative to the flag $F$ through $\eta, 22,23,28,29,35-37$

$\pi^{\mathcal{F}, \eta} \quad$ Interpretation homomorphism relative to the family of flags $\mathcal{F} \subset \mathcal{F}_{\left|\sigma_{2}\right|+1}^{\sigma_{2}}$ through $\eta$, 22,23

$\pi^{\sigma, \eta}$

$\left.\sigma\right|_{\eta}$

$\tau^{F}$

$1_{\sigma}=(\sigma, \mathrm{Id})$

0

1
Upward operator relative to type extension $(\sigma, \eta), 20-23,65$

Type induced by $\eta$ on $\sigma, 16$ Operation $\tau^{F}$ (see Definition 2.4.3), 34-39 Flag algebra unity, 9, 14-18, 20-23, 27-33, 37, 38, 62-64 Type of size $0,9,16,18,20,22-39,42,43,45,48,49,51,52,54,55,62-66$

Type of size $1,10,11,16,17,22-24,28,31,35-37,55,63-66$

$A$ Type on $T_{\text {Tournament }}$ of size 2 where the vertex labelled 1 beats the vertex labelled $2,3,4$, 26-33, 35-38, 42-48, 64

$\mathcal{A}^{\sigma}$ $\sigma$-flag algebra, 14-26, 28-37, 42, 43, 45-48, 51, 52, 54, 55, 61-66

$\mathcal{A}_{u}^{\sigma} \quad$ Localization of flag algebra $\mathcal{A}^{\sigma}$ with respect to the multiplicative system $\left\{u^{\ell}: \ell \in \mathbb{N}\right\}$, 19,20

$\operatorname{Aut}(M)$

Group of automorphisms of a model $M, 25,26,35-37,51$

$\mathcal{B}(X)$

Set of Borel sets of the topological space $X, 18$

$\mathcal{C}\left(\mathcal{F}^{\sigma}\right)$

Ordinary cone relative to $\mathcal{F}^{\sigma}, 61-63$

$\vec{C}_{3}$ 3-cycle, 26-28, 31-33, 48, 54, 63

$\vec{C}_{3}^{A}$ $A$-flag of size 3 whose underlying model is $\vec{C}_{3}^{A}, 3,4,26-28,30-33,42-48,63,64$

$\mathcal{C}_{\text {sem }}\left(\mathcal{F}^{\sigma}\right)$

Semantic cone relative to $\mathcal{F}^{\sigma}, 61-63$

E

Type of size 2 on $T_{\text {Graph }}$ corresponding to an edge, 10, 17, 22, 23

$\bar{E}$

Type of size 2 on $T_{\text {Graph }}$ corresponding to a non-edge, 10, 17, 22

$\mathbb{E}$

Expected value, 18, 23, 25, 26, 28-33, 36-38, 42, 45, 48, 55, 64

$\operatorname{Ext}(\sigma, \eta)$

Set of all types $\sigma_{2}$ such that $\left(\sigma_{2}, \eta\right)$ is a type extension of $\sigma, 21$

$\left.F\right|_{\eta}$

Restriction of flag $F$ through $\eta, 16,18,20,26,65$

$\mathcal{F}^{\sigma} \quad$ Family of all (finite) $\sigma$-flags up to isomorphism, 9, 10, 13-20, 34, 46, 48, 49, 61-63, 65 
Family of all (finite) $\sigma$-flags that are $U$-models up to isomorphism, 19

$\mathcal{F}_{\ell}^{\sigma, U} \quad$ Family of all $\sigma$-flags up to isomorphism that are $U$-models and have size $\ell, 19,22$ $\mathcal{F}_{\ell}^{\sigma} \quad$ Family of all $\sigma$-flags of size $\ell$ up to isomorphism, 9, 13-15, 17, 19, 22, 26, 29, 36-39, 43, $46,47,62,63$

$F \downarrow \eta$

$\bar{G}$

$G^{(\vec{k})}$

$G_{\tau}$

$G_{n, p}$

$\operatorname{Grad}_{\vec{M}, a}(f)$

$\operatorname{Hom}\left(\mathcal{A}^{\sigma}, \mathbb{R}\right)$
Flag removing restriction of flag $F$ through $\eta, 20$

Complement of the graph $G, 10,11,16,17,22,23,51$

Blow-up of the graph $G$ relative to vector $\vec{k}, 53$

Graph of inversions of permutatin $\tau, 8,24$

Erdős-Rényi random graph of size $n$ and edge density $p, 1,51$

Model gradient of function $f$ relative to $\vec{M}$ and point $a, 65,66$

$\operatorname{Hom}^{+}\left(\mathcal{A}^{\sigma}, \mathbb{R}\right) \quad$ Set of positive homomorphisms from $\mathcal{A}^{\sigma}$ to $\mathbb{R}, 15-18,20-23,25,26,28-37$, $42,43,45,48,51,52,54,55,61-66$

$I^{\prime}(F)$

Flag interpretation of flag $F$ by translation $I, 19,20,22$

$I(M)$ Model interpretation of model $M$ by translation $I, 19,24,59$

$I^{A} \quad$ A-flag of size 3 where the unlabelled vertex beats both labelled vertices, 3, 4, 26-28, 30, $32,34-38,43,44,46-48$

$I_{\mathrm{AP}}$

Id

$\operatorname{Id}_{k}$

$I_{\text {Inv }}$

$I_{\text {link }}$

$\operatorname{im}$

$I_{\mathrm{OE}}$

$I_{\mathrm{SOE}}$

$\mathcal{K}^{\sigma}$

$K_{n}$
Anti-parallel arc interpretation, 24

Identity function, 9, 14

Identity permutation of size $k, 52$

Inversion interpretation, 24

Link interpretation, 24

Image, 9, 16, 17

Orientation-erasing interpretation, 24

Strict orientation-erasing interpretation, 24

Kernel of the type $\sigma, 14,16,20,34,66$

Complete graph on $n$ vertices, 10, 11, 15-17, 22, 23, 51, 53, 58

$L_{4}$ Tournament on 4 vertices that has exactly one 3-cycle and one vertex with outdegree 0 , 
$27,31,32,41,43,45,53-55$

$\leqslant \sigma$

Preorder relative to type $\sigma, 61-64$

$\mathcal{M}$

Family of all finite models up to isomorphism, 66

$\left.M\right|_{W}$ Restriction of the model $M$ to the set $W, 6,7,9,14,19,20,66$

$M^{\sigma}$ Unique $\sigma$-flag over $M, 9-11,16,17,22,23$

$\mathcal{M}_{k}$ Family of all models of size $k$ up to isomorphism, 6, 9, 10, 51, 65

$\mathbb{N}=\{0,1,2, \ldots\} \quad$ Set of natural numbers, 1-4, 6-10, 13, 15, 18, 19, 25, 26, 29, 31, 33, 38, $41,42,45,46,48,49,51-53,61,62$

$O^{A} \quad A$-flag of size 3 where the unlabelled vertex is beaten by both labelled vertices, 3,4 , 26-30, 32, 34, 35, 37, 38, 43, 44, 46-48

$\mathbb{P}$

$p(\cdot ; \cdot)$

$\mathbb{P}_{F}^{\sigma, \eta}$

$P_{n}$

$\mathcal{P}_{\text {qr }}$

$q_{\sigma, \eta}(F)$

$\mathcal{Q}_{\text {qr }}$
Probability, 9, 15, 16, 21, 22, 25, 46 Joint density, 1-3, 6, 8-10, 13-15, 17, 25, 26, 41, 46, 47, 62, 63, 66 Probability measure extending $F$ to $\sigma$ through $\eta, 17,18,46,47$ Path on $n$ vertices, 10, 11, 16, 17, 22, 23 Set of minimization quasi-random characterizers, 33, 34, 37, 38 Set of quasi-randomly minimized elements, 33, 34, 36-38

$\mathbb{R} \quad$ Field of real numbers, 14-18, 20-23, 25, 26, 28-37, 42, 43, 45, 48, 51, 52, 54, 55, 61-66 $R_{2 n+1}$ Carousel tournament of order $2 n+1,4,42,45-49,53$

$R_{4}$ Tournament on 4 vertices that has a 4-cycle, 3, 4, 26, 27, 31-33, 38, 42-45, 48, 53, 55, 64 $\operatorname{Rev}_{k}$ Reverse permutation of size $k, 52$ $\boldsymbol{R}_{n, 1 / 2}$ Random tournament of size $n, 2,25,26,53$

$\mathbb{R} X \quad$ Set of formal $\mathbb{R}$ linear combinations of elements of $X, 13,14,16,19,34,36,37,66$

$\mathfrak{S}$

$\mathfrak{S}[X]$

$\mathfrak{S}_{k}$
Family of all permutations over $[k]$ for some $k \in \mathbb{N}, 7$ Family of all permutations over the set $X, 7$ Family of all permutations over $[k], 7,8,10,52$ Extension of the theory $T$ by the type $\sigma, 9,14,19,24$ 
$T_{\text {Digraph }}$

$T_{\text {Graph }}$

$T_{\text {Graphofinversions }}$

$T_{k \text {-hypergraph }}$

$T_{\text {Perm }}$
Theory of Digraphs, 6, 24, 64 Theory of Graphs, 5, 6, 10, 23, 24, 51, 58, 64, 66 Theory of Graphs of Inversions, 8, 24 Theory of $k$-uniform hypergraphs, 8,24 Theory of Permutations, 8, 10, 24, 51, 52, 64

$\operatorname{Tr}_{3}^{A} \quad A$-flag of size 3 where the unlabelled vertex is beaten by the vertex labelled 1 and beats the vertex labelled $2,3,4,26-28,30,32-39,43,44,46-48,64$

$\operatorname{Tr}_{3}^{\mathrm{W}} \quad$ 1-flag obtained from $\operatorname{Tr}_{3}$ by labelling the vertex with maximum outdegree, 28

$\operatorname{Tr}_{4}^{A} \quad A$-flag obtained from $\operatorname{Tr}_{4}$ by labelling the vertex with maximum outdegree with 1 and the vertex with minimum outdegree with 2,30

$\operatorname{Tr}_{k} \quad$ Transitive tournament of size $k, 2,3,26-34,37,38,43-45,48,53,55,63,64$

$\operatorname{Tr}_{k}^{\mathrm{W} 2} \quad$ A-flag obtained from $\operatorname{Tr}_{k}$ by labelling the vertex with maximum outdegree with 1 and the vertex with second largest outdegree with 2, 28

$T_{\text {Tournaments }}$

Theory of Tournaments, $6,10,24,25,41,45,64$

$V(M)$ Set of vertices of the model $M, 1-3,5-9,14,16,17,19-21,24,25,35,38,41,42,53$, $57-59,66$

Var

Variance, 3, 32, 33, 44

$W_{4}$ Tournament on 4 vertices that has exactly one 3-cycle and one vertex with outdegree 3 , $27,31,32,41,43,45,53-55$ 


\section{List of figures}

1.1 Models, types and flags in the Theory of graphs. . . . . . . . . . . . . . . . 10

2.1 Tournaments of sizes 3 and 4, type $A$ and $A$-flags of size $3 . \ldots 27$

2.2 Type 1 and flags of size 2 over the type $1 \ldots \ldots \ldots$

3.1 Neighbourhoods of $\boldsymbol{\theta}(1)$ and $\boldsymbol{\theta}(2) \ldots \ldots \ldots \ldots$. . . . . . . . . . . 47

4.1 Typical structure of the random tournament $\boldsymbol{S}_{N, t} \ldots \ldots \ldots \ldots$ 


\section{List of tables}

1.1 Joint densities in the Theory of graphs. . . . . . . . . . . . . . . . . . 11

1.2 Joint densities in the Theory of permutations (type 0 ). . . . . . . . . . 11

1.3 Joint densities in the Theory of permutations (type 1 ). . . . . . . . . . . 12

1.4 Downward operator in the Theory of graphs (type 1) . . . . . . . . 16

1.5 Downward operator in the Theory of graphs (type $E$ ). . . . . . . . . . 17

1.6 Downward operator in the Theory of graphs (type $\bar{E})$. . . . . . . . . . . 17

2.1 Joint densities in the Theory of tournaments (type 0 ) . . . . . . . . . . 27

2.2 Downward operator in the Theory of tournaments. . . . . . . . . . . 28

3.1 Comparison of quasi-random properties and quasi-carousel properties. . . . . 44 


\section{Introduction}

One of the most interesting measurements one can make of a large combinatorial object $N$ is to compute the density of a small fixed template object $M$ with the same signature in $N$ (denoted by $p(M ; N)$ ). Although a precise calculation of this measure requires testing $\left(\begin{array}{l}|V(N)| \\ |V(M)|\end{array}\right)=\Omega\left(|V(N)|^{|V(M)|}\right)$ sets, a good approximation of $p(M ; N)$ can be made by repeatedly (and independently) picking a subset of $V(N)$ with cardinality $|V(M)|$ uniformly at random and computing the empirical probability that this set induces an object isomorphic to $M$. Another good property of this measurement is its stability with respect to small changes in the large object $N$, that is, if $L$ is obtained from $N$ by making changes (e.g. vertex addition or removal, predicate ${ }^{1}$ editing) that are small compared to the $\operatorname{size}^{2,3}|V(N)|$ of $N$, then the density of $M$ in $L$ is close to the density of $M$ in $N$.

This suggests a comparison framework for large objects, or rather for sequences of objects whose sizes tend to infinity, through the density of fixed templates. More precisely, we expect that if two sequences of combinatorial objects $\left(N_{n}\right)_{n \in \mathbb{N}}$ and $\left(L_{n}\right)_{n \in \mathbb{N}}$ are such that

$$
\forall n \in \mathbb{N},\left|V\left(N_{n}\right)\right|=\left|V\left(L_{n}\right)\right|<\left|V\left(N_{n+1}\right)\right| \quad \lim _{n \rightarrow \infty} p\left(M ; N_{n}\right)-p\left(M ; L_{n}\right)=0 ;
$$

for every fixed object $M$; then they should be very similar.

This notion of similarity of sequences of objects can be traced back to the theory of graph quasi-randomness, whose main object of study is the Erdös-Rényi random graph $\boldsymbol{G}_{\boldsymbol{n}, \boldsymbol{p}}$ of size $n$, in which each edge is present with probability $p$ independently of all other edges. This theory was originated in the seminal papers by Thomason [Tho87] and Chung, Graham and Wilson [CGW89] (see also [KS06] for a survey) and its main thrust is that several a priori different properties that a sequence of graphs $\left(G_{n}\right)_{n \in \mathbb{N}}$ of increasing sizes may have are actually equivalent and equivalent to $\left(G_{n}\right)_{n \in \mathbb{N}}$ being similar to the sequence of Erdős-Rényi random graphs, that is, equivalent to

$$
\lim _{n \rightarrow \infty} p\left(H ; G_{n}\right)-p\left(H ; \boldsymbol{G}_{\left|\boldsymbol{V}\left(\boldsymbol{G}_{\boldsymbol{n}}\right)\right|, \boldsymbol{p}}\right)=0,
$$

for every fixed graph $H$. The theory of quasi-randomness has developed into a vast field with branches in several other theories, such as uniform hypergraphs [BR13, Chu12, CG90], graph orientations [Gri13], permutations [Coo04, KP13], and tournaments [CG91, KS13].

From this notion of similarity of sequences we can also define a very useful notion of convergence, namely, we say that a sequence of combinatorial objects $\left(N_{n}\right)_{n \in \mathbb{N}}$ of increasing sizes is convergent if $\left(p\left(M ; N_{n}\right)\right)_{n \in \mathbb{N}}$ is convergent for every fixed object $M$. With this notion of convergence, one can define limit objects that codify the limits of these sequences of densities.

\footnotetext{
${ }^{1}$ For instance, in the Theory of Graphs, edges are binary predicates.

${ }^{2}$ In classical combinatorics, we usually call $|V(N)|$ the order of $N$, but here we choose to use size in conformity with flag algebra nomenclature.

${ }^{3}$ Or rather, in the case of $k$-ary predicate editing, compared to $|V(N)|^{k}$.
} 
One approach is to define the limit objects to be semantically close to the underlying combinatorial objects, that is, to find a limit object that resembles some representation of the combinatorial objects (such approach was first taken by Lovász and Szegedy by defining graphons [LS06] and has also been taken in the definitions of hypergraphons [ES12], permutons $\left[\mathrm{HKM}^{+} 13\right]$, and digraphons [DJ08, Section 9]). The clear advantage of this approach is that operations and proofs involving these limit objects have intuitive analogous versions in the finite world.

Another approach is to study the convergent sequences syntactically, that is, to study what kind of properties the sequence of numbers $(\phi(M))_{M}$ must satisfy if we have $\phi(M)=$ $\lim _{n \rightarrow \infty} p\left(M ; N_{n}\right)$. This latter approach is precisely the thrust of the theory of flag algebras [Raz07], whose main advantages are its generality — in the sense that it constructs the limit object of any universal theory $T$ - and the simplicity of the arguments in its proofs ${ }^{4}$.

A first and trivial observation about this sequence $(\phi(M))_{M}=\left(\lim _{n \rightarrow \infty} p\left(M ; N_{n}\right)\right)_{M}$ is that each of its coordinates is a number in the interval $[0,1]$. Taking this one step ahead, we see that actually some coordinates are limited to an even smaller set; for instance, in the Theory of Tournaments, if $T$ is a sufficiently large tournament, then it cannot be that no triple of vertices induces a transitive tournament ${ }^{5}$. A natural problem that arises is then the following.

Problem. Given a fixed object $L$, what is the minimum and the maximum value of $\phi(L)$ subject to $(\phi(M))_{M}=\left(\lim _{n \rightarrow \infty} p\left(M ; N_{n}\right)\right)_{M}$ for a sequence of objects $\left(N_{n}\right)_{n \in \mathbb{N}}$ of increasing sizes?

Furthermore, if $\left(N_{n}\right)_{n \in \mathbb{N}}$ is a sequence of objects of increasing sizes that attains this minimum (or maximum), then what does this sequence look like?

This translates into the following classical extremal combinatorics problem.

Problem. Given a fixed object $M$, compute the following extremal values.

$$
\begin{aligned}
& \inf \left\{\liminf _{n \rightarrow \infty} p\left(M ; N_{n}\right):\left(N_{n}\right)_{n \in \mathbb{N}} \text { is a sequence of objects with } \lim _{n \rightarrow \infty}\left|V\left(N_{n}\right)\right|=\infty\right\} ; \\
& \sup \left\{\limsup _{n \rightarrow \infty} p\left(M ; N_{n}\right):\left(N_{n}\right)_{n \in \mathbb{N}} \text { is a sequence of objects with } \lim _{n \rightarrow \infty}\left|V\left(N_{n}\right)\right|=\infty\right\} .
\end{aligned}
$$

Furthermore, if $\left(N_{n}\right)_{n \in \mathbb{N}}$ is an extremal sequence, that is, if $\lim _{n \rightarrow \infty} p\left(M ; N_{n}\right)$ equals the infimum (or supremum) above, then what does $N_{n}$ look like?

Going back to our tournament example, since the transitive tournament $\operatorname{Tr}_{k}$ of size $k$ has a very organized structure, a natural guess for an extremal sequence for the minimization problem would be the sequence of random tournaments $\left(\boldsymbol{R}_{\boldsymbol{n}, \mathbf{1 / 2}}\right)_{n \in \mathbb{N}}$, where $\boldsymbol{R}_{\boldsymbol{n}, \mathbf{1 / 2}}$ is the random tournament of size $n$ in which each arc orientation has probability $1 / 2$ independently of other pairs of vertices.

The first original result of this dissertation (Theorem 2.2.2), obtained in joint work with Alexander A. Razborov, says that not only does the sequence $\left(\boldsymbol{R}_{\boldsymbol{n , \mathbf { 1 } / \mathbf { 2 }}}\right)_{n \in \mathbb{N}}$ minimize the density of the transitive tournament of size $k$, but also it is the only minimizer for $k \geqslant 4$ in the following sense: if $\left(T_{n}\right)_{n \in \mathbb{N}}$ is a sequence of tournaments of increasing sizes minimizing the density of $\operatorname{Tr}_{k}$, then $\left(T_{n}\right)_{n \in \mathbb{N}}$ is quasi-random, that is, we have

$$
\lim _{n \rightarrow \infty} p\left(T ; T_{n}\right)-p\left(T ; \boldsymbol{R}_{\left|\boldsymbol{V}\left(\boldsymbol{T}_{n}\right)\right|, \mathbf{1} / \mathbf{2}}\right)=0,
$$

for every fixed tournament $T$.

\footnotetext{
${ }^{4}$ Perhaps only once you get used to its notation.

${ }^{5}$ In fact, a tournament $T$ of size at least 4 already has 3 -transitive tournament density at least $1 / 2$.
} 
With the same proof techniques, we also generalize this result to several formal linear combinations of tournaments (Theorem 2.4.9), that is, we obtain several other $\sum_{i} c_{i} L_{i}$ such that $\left(T_{n}\right)_{n \in \mathbb{N}}$ minimizes

$$
\lim _{n \rightarrow \infty} p\left(\sum_{i} c_{i} L_{i} ; T_{n}\right)=\lim _{n \rightarrow \infty} \sum_{i} c_{i} p\left(L_{i} ; T_{n}\right)
$$

if and only if $\left(T_{n}\right)_{n \in \mathbb{N}}$ is quasi-random.

Perhaps two of the most interesting quasi-random properties concern what we call in the language of flag algebras $A$-flag density concentration. Given an arc $u v \in A(T)$ of a tournament $T$, all other vertices $w \in V(T)$ can be classified into four classes (" $A$-flags"):

- $u w, v w \in A(T)$;

- $w u, w v \in A(T)$

- $u w, w v \in A(T)$;

- $w u, v w \in A(T)$.

Let $O^{A}(u, v), I^{A}(u, v), \operatorname{Tr}_{3}^{A}(u, v)$ and $\vec{C}_{3}^{A}(u, v)$ denote the number of vertices in these four classes divided by $|V(T)|-2$ respectively. One keystone quasi-random property $\left(P_{4}\right.$, to be exact) says that a sequence of tournaments $\left(T_{n}\right)_{n \in \mathbb{N}}$ is quasi-random if and only if

$$
\lim _{n \rightarrow \infty} O^{A}\left(\boldsymbol{u}_{\boldsymbol{n}}, \boldsymbol{v}_{\boldsymbol{n}}\right)=1 / 4 \text { a.s., }
$$

where $\boldsymbol{u}_{\boldsymbol{n}} \boldsymbol{v}_{\boldsymbol{n}}$ is an arc of $T_{n}$ chosen uniformly at random. By flipping all arcs, it is easy to see that an analogous property with $I^{A}$ is also a quasi-random property.

As a byproduct of the first result we are able to prove (Theorem 2.3.1) that the two other analogous properties (concerning $\operatorname{Tr}_{3}^{A}$ and $\vec{C}_{3}^{A}$ ) are also quasi-random properties. In fact, we can even get rid of the $1 / 4$ in the first three classes, that is, we prove (Theorem 2.3.6) that if $F \in\left\{O^{A}, I^{A}, \operatorname{Tr}_{3}^{A}\right\}$, then the property

$$
\lim _{n \rightarrow \infty} \operatorname{Var}\left[F\left(\boldsymbol{u}_{\boldsymbol{n}}, \boldsymbol{v}_{\boldsymbol{n}}\right)\right]=0
$$

is a quasi-random property. For the final class, we prove (Theorem 2.3.7) that if

$$
\lim _{n \rightarrow \infty} \operatorname{Var}\left[\vec{C}_{3}^{A}\left(\boldsymbol{u}_{\boldsymbol{n}}, \boldsymbol{v}_{\boldsymbol{n}}\right)\right]=0,
$$

then the sequence $\left(T_{n}\right)_{n \in \mathbb{N}}$ either is quasi-random or is similar to the sequence of transitive tournaments ${ }^{6}\left(\operatorname{Tr}_{n}\right)_{n \in \mathbb{N}}$.

Noting that the result on minimization of transitive tournaments closes the non-trivial cases of minimization of a single tournament (Corollary 2.2.5), we shift our attention to the maximization problem in the Theory of Tournaments.

The second original result of this dissertation concerns maximization of the tournament $R_{4}$ (which is the only tournament on 4 vertices containing a 4-cycle) and what we call quasicarousel properties.

As one may imagine, the quasi-carousel properties are a set of a priori different properties that a sequence of tournaments $\left(T_{n}\right)_{n \in \mathbb{N}}$ of increasing sizes may have and that are

\footnotetext{
${ }^{6}$ One might perhaps call such sequences quasi-transitive.
} 
actually equivalent and equivalent to $\left(T_{n}\right)_{n \in \mathbb{N}}$ being similar to a certain sequence of tournaments $\left(R_{2 n+1}\right)_{n \in \mathbb{N}}$ (we call the tournaments of this sequence carousel tournaments).

One of the quasi-carousel properties is the maximization of the density of $R_{4}$ and we also present several quasi-carousel properties that have nice analogies in quasi-randomness. For instance, the quasi-carousel properties analogous to the $A$-flag concentration in quasirandomness are the following: if $F$ is one of $O^{A}, I^{A}, \operatorname{Tr}_{3}^{A}$ or $\vec{C}_{3}^{A}$, then quasi-carousel Property $S_{5}(\mathrm{~F}, 1 / 2)$ of a sequence of tournaments $\left(T_{n}\right)_{n \in \mathbb{N}}$ says that if $\boldsymbol{u}_{\boldsymbol{n}} \boldsymbol{v}_{\boldsymbol{n}}$ is an arc of $T_{n}$ chosen uniformly at random, then $F\left(\boldsymbol{u}_{\boldsymbol{n}}, \boldsymbol{v}_{\boldsymbol{n}}\right)$ converges in distribution to a uniform random variable on $[0,1 / 2]$.

The text is organized as follows. In Chapter 1, we introduce the theory of flag algebras, presenting its basic definitions and properties, and aiming to present its inductive method, which is the main technique in the proofs of the subsequent chapters. In Chapter 2, we present the results related to minimization of transitive tournaments and quasi-randomness. In Chapter 3, we present the results related to quasi-carouselness. In Chapter 4, we briefly comment the novel results of the previous chapters and state some related questions and conjectures. Appendix A contains some definitions, notations and properties on first-order logic (that may not be of the common folklore) and Appendix B contains some proof techniques of flag algebras, namely the semidefinite and differential methods, that are not used in the proofs presented in this work but are nevertheless important and interesting. 


\section{Chapter 1}

\section{Flag algebras}

The aim of this chapter is to introduce the reader to the theory of flag algebras developed by Razborov [Raz07] and in doing so we choose to take an intuitive approach rather than a more formal one. Throughout this text we will follow the notation of [Raz07] so the accustomed reader is free to skip this chapter. We remark that we do not follow the order of [Raz07] when presenting the concepts and properties of flag algebras ${ }^{1}$. This chapter also assumes the reader to be familiar with basic notions of first-order logic (we provide, however, some definitions and properties on the matter in Appendix A and every first appearance of these terms on the text will methodically be in an emphasized font).

Finally, we refer the reader to [Raz13b] for a shorter and (even more) intuitive introduction to the theory of flag algebras and to [Raz13a] for a survey of results using this theory.

\subsection{Scope}

The theory of flag algebras can be applied to any combinatorial structure that can be described by a universal theory in a finite first-order language with equality and without constant or function symbols. Its main goal is to study densities of a combinatorial object in another (much larger) combinatorial object. In this section, we define the notion of density in an arbitrary universal theory and present some typical combinatorial structures that can be formulated as universal theories. We also present some basic properties regarding first-order formulations.

We start with three classic examples of combinatorial structures and their first-order formulations.

Example 1.1.1. The Theory of Graphs $T_{\text {Graph }}$ can be formulated in a language with a binary predicate symbol e that codifies adjacency between two vertices, i.e., if $v$ and $w$ are two vertices of a graph $G$ (that is, two elements of the set of vertices $V(G)$ of the model $G$ of $\left.T_{\text {Graph }}\right)$, then $v w$ is an edge of $G$ if and only if $G \models e(v, w)$. The axioms of $T_{\text {Graph }}$ are the following.

i. $\forall x, \neg e(x, x)$;

ii. $\forall x \forall y,(e(x, y) \Longrightarrow e(y, x))$.

If $G$ is a model of $T_{\text {Graph }}$, that is, if $G$ is a graph, then the set of edges of $G$ is $E(G)=$ $\{\{v, w\} \subset V(G): G \models e(v, w)\}$.

\footnotetext{
${ }^{1}$ But we attempt to provide references specific to the level of sections and theorems so the interested reader can easily find them in [Raz07].
} 
Note that axiom (i) enforces that the graphs do not have loops and axiom (ii) enforces the symmetry of the adjacency relation. By removing the symmetry axiom, we obtain the Theory of Digraphs below.

Example 1.1.2. The Theory of Digraphs $T_{\text {Digraph }}$ can be formulated in a language with a binary predicate symbol $a$ where, if $v$ and $w$ are two vertices of the digraph $D$, then $v w$ is an arc of $D$ if and only if $D \models a(v, w)$. The theory $T_{\text {Digraph }}$ has only the following axiom.

i. $\forall x, \neg a(x, x)$.

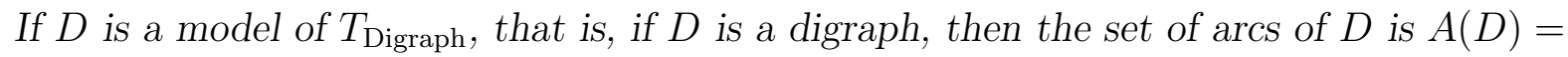
$\left\{(v, w) \in V(D)^{2}: D \models a(v, w)\right\}$.

The Theory of Digraphs can be further restricted to obtain the Theory of Tournaments, i.e., the theory of digraphs $D$ such that for every pair of distinct vertices $v, w \in V(D)$, exactly one arc $v w$ or $w v$ is in $A(D)$.

Example 1.1.3. The Theory of Tournaments $T_{\text {Tournaments }}$ can be formulated in a language with a binary predicate symbol $a$ with the following axioms.

i. $\forall x, \neg a(x, x)$;

ii. $\forall x \forall y,(x \neq y \Longrightarrow a(x, y) \underline{\vee} a(y, x))$.

Let us fix until the end of this chapter a universal theory $T$ in a finite first-order language $\{=\} \cup L$ with equality $=$ and without constant or function symbols. Let us also assume that $T$ has at least one infinite model. In fact, from here onward, unless explicitly said otherwise, all universal theories will be in a finite first-order language with equality and without constant or function symbols. Whenever we work with more than one theory, we shall append $[T]$ to the notation accordingly to avoid ambiguity.

Note that the fact that $T$ is universal without constant or function symbols implies that if $M \models T$ and $W \subset V(M)$, then the model restriction $\left.M\right|_{W}$ of $M$ to $W$ is a model of $T$, called model induced by $W$ in $M$ (see Proposition A.4).

This along with the existence of an infinite model of $T$ implies that $T$ has at least one model of each finite size. Let us denote the size of a model $M$ by $|M|=|V(M)|$, denote the family of all models of size $k$ up to isomorphism by $\mathcal{M}_{k}$ and denote the family of all finite models up to isomorphism by $\mathcal{M}=\bigcup_{k \in \mathbb{N}} \mathcal{M}_{k}$.

With the notion of model restriction, we can easily define the notions of submodel and submodel density below.

Definition 1.1.4. Let $M$ and $N$ be models of $T$.

An occurrence of $M$ in $N$ is a set $W \subset V(N)$ with $|W|=|M|$ such that $\left.N\right|_{W} \cong M$.

If $|M| \leqslant|N|<\infty$, then we define the submodel density (or density) of $M$ in $N$ as the number of occurrences of $M$ in $N$ divided by $\left(\begin{array}{l}|N| \\ |M|\end{array}\right)$, and we denote it by $p(M ; N)$.

If there exists an occurrence of $M$ in $N$, then we say that $M$ is a submodel of $N$.

Remark 1.1.5. The quantity $p(M ; N)$ can be seen as the probability that a random subset of $V(N)$ of size $|M|$ is an occurrence of $M$ in $N$.

We also remark that the notion of submodel corresponds to the notions of induced subgraph, induced subdigraph and (induced) subtournament in the theories $T_{\text {Graph }}, T_{\text {Digraph }}$ and $T_{\text {Tournaments }}$ respectively.

Note that the fact that every model restriction of a model is also a model implies that

$$
\sum_{M \in \mathcal{M}_{k}} p(M ; N)=1
$$


for every $k \leqslant|N|$.

Another typical example of universal theory is obtained by forbidding a family of finite submodels from another universal theory.

Example 1.1.6. Let $\mathcal{F}$ be a family of finite models of $T$. The theory of the models $M$ of $T$ such that no member of $\mathcal{F}$ is a submodel of $M$ can be formulated as a universal theory by appending to $T$ the axiom

$$
\forall x_{1} \forall x_{2} \cdots \forall x_{k} \neg \phi_{N}
$$

for each $N \in \mathcal{F}$, where $\phi_{N}$ is an open diagram of $N$ over $x_{1}, x_{2}, \ldots, x_{k}($ and $k=|N|)$.

The next proposition gives us an easier characterization of combinatorial structures that can be formulated as universal theories.

Proposition 1.1.7. Suppose $T^{\prime}$ is a theory in a finite first-order language $L$ and suppose that for every model $M$ of $T^{\prime}$ and every $W \subset V(M)$, the model restriction $\left.M\right|_{W}$ is a model of $T^{\prime}$. Then $T^{\prime}$ can be reformulated as a universal theory, that is, there exists a universal theory $T$ over $L$ such that for every model $M$ over $L$, we have $M \models T \Longleftrightarrow M \models T^{\prime}$.

Proof. Let $\mathcal{F}$ be the family of all finite models over $L$ that are not models of $T^{\prime}$ and consider the theory $T$ over $L$ whose axioms are of the form

$$
\forall x_{1} \forall x_{2} \cdots \forall x_{k} \neg \phi_{M}
$$

for $M \in \mathcal{F}$ and where $\phi_{M}$ is an open diagram of $M$ over $x_{1}, x_{2}, \ldots, x_{k}$.

Trivially $T$ is universal and every model of $T$ is a model of $T^{\prime}$.

On the other hand, if $M$ is a model of $T^{\prime}$, then every model restriction of $M$ is also a model of $T^{\prime}$, hence not an element of $\mathcal{F}$. Therefore $M$ must also be a model of $T$.

Remark 1.1.8. Informally, Proposition 1.1.7 says that a theory $T$ is universal if and only if every model restriction of $T$ is a model of $T$.

Note, however, that the formulation of $T$ given in Proposition 1.1.7 is not necessarily effective (in the sense that its axioms can be recursively enumerated), but this is not important for us here.

Perhaps the first non-trivial example of combinatorial structure that can be formulated as a universal theory is the Theory of Permutations below.

Example 1.1.9. A permutation over a set $X$ is a bijective function $\tau: X \rightarrow X$. We denote the set of all permutations over $X$ by $\mathfrak{S}[X]$.

We are particularly interested in the case when $X=[k]=\{1,2, \ldots, k\}$, so we define $\mathfrak{S}_{k}=$ $\mathfrak{S}[[k]]$. We also define $\mathfrak{S}=\bigcup_{k \in \mathbb{N}} \mathfrak{S}_{k}$.

Every permutation $\tau \in \mathfrak{S}_{k}$ induces an order $<_{\tau}$ over $[k]$ called order induced by the permutation $\tau$ and defined by

$$
i<_{\tau} j \Longleftrightarrow \tau(i)<\tau(j)
$$

for every $i, j \in[k]$.

If $\tau \in \mathfrak{S}_{k}$ and $\eta \in \mathfrak{S}_{m}$, then an occurrence of $\tau$ in $\eta$ is a set $S=\left\{s_{1}, s_{2}, \ldots, s_{k}\right\} \subset[m]$ with $s_{1}<s_{2}<\cdots<s_{k}$ such that for every $i, j \in[k]$, we have

$$
i<_{\tau} j \Longleftrightarrow s_{i}<_{\eta} s_{j}
$$

The density of $\tau$ in $\eta$ is the number of occurrences of $\tau$ in $\eta$ divided by $\left(\begin{array}{c}m \\ k\end{array}\right)$. 
The Theory of Permutations (over $[k]$ for some $k \in \mathbb{N}$ ) $T_{\text {Perm }}$ can be formulated in a language with two binary predicate symbols $a$ and $b$ both with the axioms of total order, that is, we have the following axioms.

$$
\begin{aligned}
& \text { i. } \forall x \forall y, a(x, y) \vee a(y, x) \text {; } \\
& \text { ii. } \forall x \forall y,(a(x, y) \wedge a(y, x) \Longrightarrow x=y) \text {; } \\
& \text { iii. } \forall x \forall y \forall z,(a(x, y) \wedge a(y, z) \Longrightarrow a(x, z)) \text {; } \\
& \text { iv. } \forall x \forall y, b(x, y) \vee b(y, x) \text {; } \\
& \text { v. } \forall x \forall y,(b(x, y) \wedge b(y, x) \Longrightarrow x=y) \text {; } \\
& \text { vi. } \forall x \forall y \forall z,(b(x, y) \wedge b(y, z) \Longrightarrow b(x, z)) \text {. }
\end{aligned}
$$

Every permutation $\tau \in \mathfrak{S}_{k}$ corresponds to the model over $[k]$ where $a$ and $b$ are respectively interpreted as the natural order $<$ over $[k]$ and the order $<_{\tau}$. The notions of occurrence and density of Definition 1.1.4 coincide with the ones defined in this example.

Now we present a non-trivial example of a theory which can be easily seen to be universal in light of Proposition 1.1.7.

Example 1.1.10. The graph of inversions of a permutation $\tau \in \mathfrak{S}_{k}$, denoted by $G_{\tau}$, is given by

$$
\begin{aligned}
& V\left(G_{\tau}\right)=[k] \\
& E\left(G_{\tau}\right)=\left\{\{i, j\} \subset[k]: i<j \wedge j<_{\tau} i\right\} .
\end{aligned}
$$

The Theory of Graphs of Inversions is denoted by $T_{\text {Graphofinversions }}$.

Clearly any induced subgraph of a graph of inversions (of some permutation) is a graph of inversions (of some permutation), hence $T_{\text {Graphofinversions }}$ can be formulated as a universal theory.

Finally, let us present a theory with a predicate of higher arity.

Example 1.1.11. The Theory of $k$-uniform hypergraphs $T_{k \text {-hypergraph }}$ can be formulated in a language with a $k$-ary predicate symbol $e$ where, if $v_{1}, v_{2}, \ldots, v_{k}$ are vertices of the hypergraph $\mathcal{H}$, then $v_{1} v_{2} \cdots v_{k}$ is a hyperedge of $\mathcal{H}$ if and only if $\mathcal{H} \models e\left(v_{1}, v_{2}, \ldots, v_{k}\right)$. The

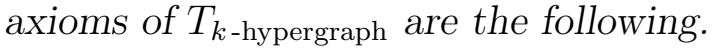

$$
\begin{aligned}
& \text { i. } \forall x_{1} \forall x_{2} \cdots \forall x_{k-1}, \neg e\left(x_{1}, x_{1}, x_{2}, x_{3}, \ldots, x_{k-1}\right) \text {; } \\
& \text { ii. } \forall x_{1} \forall x_{2} \cdots \forall x_{k},\left(\bigwedge_{\sigma \in \mathfrak{S}_{k}} e\left(x_{\sigma(1)}, x_{\sigma(2)}, \ldots, x_{\sigma(k)}\right)\right) \underline{\vee}\left(\bigwedge_{\sigma \in \mathfrak{S}_{k}} \neg e\left(x_{\sigma(1)}, x_{\sigma(2)}, \ldots, x_{\sigma(k)}\right)\right) \text {. }
\end{aligned}
$$

If $\mathcal{H}$ is a model of $T_{k \text {-hypergraph }}$, that is, if $\mathcal{H}$ is a $k$-uniform hypergraph, then the set of hyperedges of $\mathcal{H}$ is

$$
E(\mathcal{H})=\left\{W=\left\{w_{1}, w_{2}, \ldots, w_{k}\right\} \subset V(\mathcal{H}): \mathcal{H} \models e\left(w_{1}, w_{2}, \ldots, w_{k}\right)\right\} .
$$

\subsection{Basic definitions}

In this section, we will present the basic definitions and properties of flag algebras.

As we mentioned in Section 1.1, the main goal of the flag algebra theory is to study the quantity $p(M ; N)$ when $N$ is much larger than $M$. However, to prove even simple statements 
about these quantities, we often need to analyse a more general notion of density in which some vertices of $M$ must be mapped to some prescribed values in $V(N)$. To make this notion more precise, we first need some definitions.

Definition 1.2.1 ([Raz07, Section 2.1]). A type in the universal theory $T$ is a model $\sigma$ of $T$ such that $V(\sigma)=[k]$, for some $k \in \mathbb{N}(k=|\sigma|)$.

Let $\sigma$ be a type of size $k$. We extend the language $L$ to $L\left(c_{1}, c_{2}, \ldots, c_{k}\right)$ by appending new constant symbols $c_{1}, c_{2}, \ldots, c_{k}$ and define the extension of the theory $T$ by the type $\sigma$ as the universal theory $T^{\sigma}$ over the language $L\left(c_{1}, c_{2}, \ldots, c_{k}\right)$ obtained by appending the open diagram of $\sigma$ over $c_{1}, c_{2}, \ldots, c_{k}$ to the axioms of $T$.

We also define a $\sigma$-flag as a finite model $M$ of $T$ partially labelled with $[k]$ in a way that the labelling is a model embedding of $\sigma$ in $M$. Formally, a $\sigma$-flag is a pair $(M, \theta)$ such that $M$ is a finite model of $T$ and $\theta:[k] \rightarrow V(M)$ is a model embedding of $\sigma$ in $M$.

The size of the $\sigma$-flag $F=(M, \theta)$ is defined as $|F|=|M|$.

Note that there exists a unique type of size 0 and it will be denoted by 0 for convenience.

Note also that a $\sigma$-flag can be alternatively seen as a finite model of the theory $T^{\sigma}$ and as such we borrow some notation and nomenclature from this theory.

Definition 1.2.2 ([Raz07, Section 2.1]). If $\sigma$ is a type of size $k$, we will often denote a $\sigma$-flag $F=(M, \theta)$ by explicitly listing the values of $\theta$ in the form $(M, \theta(1), \theta(2), \ldots, \theta(k))$.

If $F=(M, \theta)$ and $F^{\prime}=\left(M^{\prime}, \theta^{\prime}\right)$ are $\sigma$-flags, we define a flag embedding of $F$ in $F^{\prime}$ as a model embedding $\alpha$ of $T^{\sigma}$, that is, the function $\alpha: V(M) \rightarrow V\left(M^{\prime}\right)$ is a model embedding of $T$ such that $\alpha \circ \theta=\theta^{\prime}$ (i.e., it preserves labelling). Furthermore, we say that $F$ and $F^{\prime}$ are isomorphic (denoted by $F \cong F^{\prime}$ ) if they are isomorphic as models of $T^{\sigma}$, that is, if there exists a bijective flag embedding of $F$ in $F^{\prime}$.

Note that if $F=(M, \theta)$ is a $\sigma$-flag and $W \subset V(M)$ is such that the image $\operatorname{im}(\theta)$ of $\theta$ is contained in $W$, then $\left.F\right|_{W}=\left(\left.M\right|_{W}, \theta\right)$ is also a model of $T^{\sigma}$, i.e., a $\sigma$-flag.

Let us denote the family of all $\sigma$-flags of size $\ell$ up to isomorphism by $\mathcal{F}_{\ell}^{\sigma}$ and denote the family of all (finite) $\sigma$-flags up to isomorphism by $\mathcal{F}^{\sigma}$.

Note that $\mathcal{M}_{\ell}$ can be naturally identified with $\mathcal{F}_{\ell}^{0}$. Furthermore, note that $\mathcal{F}_{|\sigma|}^{\sigma}$ has exactly one element, which will be denoted by $1_{\sigma}$ and is given by $1_{\sigma}=(\sigma$, Id), where Id is the identity automorphism of $\sigma$.

When there exists a model embedding of a type $\sigma$ in a model $M$ and all $\sigma$-flags resulting from such model embeddings are isomorphic, we will denote such $\sigma$-flag by $M^{\sigma}$.

We are now in condition of defining the more general notion of density called joint density.

Definition 1.2.3 ([Raz07, Definition 1]). Let $\sigma$ be a type of size $k$, let $\ell, \ell_{1}, \ell_{2}, \ldots, \ell_{t} \geqslant k$ be integers such that

$$
\left(\sum_{i=1}^{t} \ell_{i}\right)-(t-1) k \leqslant \ell,
$$

and let $F=(M, \theta), F_{1}, F_{2}, \ldots, F_{t} \in \mathcal{F}^{\sigma}$ be $\sigma$-flags of sizes $\ell, \ell_{1}, \ell_{2}, \ldots, \ell_{t}$ respectively.

The joint density of $F_{1}, F_{2}, \ldots, F_{t}$ in the flag $F$ (denoted by $p\left(F_{1}, F_{2}, \ldots, F_{t} ; F\right.$ )) is defined through the following random experiment. We pick uniformly at random pairwise disjoint subsets $\boldsymbol{W}_{\mathbf{1}}, \boldsymbol{W}_{\mathbf{2}}, \ldots, \boldsymbol{W}_{\boldsymbol{t}}$ of $V(F) \backslash \operatorname{im}(\theta)$ subject to $\left|\boldsymbol{W}_{\boldsymbol{i}}\right|=\ell_{i}-k$ for every $i \in[t]$ (whose existence is guaranteed by inequality (1.1)) and set

$$
p\left(F_{1}, F_{2}, \ldots, F_{t} ; F\right)=\mathbb{P}\left[\forall i \in[t],\left.F\right|_{\operatorname{im}(\theta) \cup \boldsymbol{W}_{\boldsymbol{i}}} \cong F_{i}\right] .
$$


Furthermore, if $F_{1}, F_{2} \in \mathcal{F}^{\sigma}$ are $\sigma$-flags such that $p\left(F_{1} ; F_{2}\right)>0$, then we say that $F_{1}$ is a subflag of $F_{2}$.

Throughout this chapter, we will mainly use as examples the theories $T_{\text {Graph }}$ and $T_{\text {Perm }}$. A third example with $T_{\text {Tournaments }}$ can be found in Chapter 2 (see Definitions 2.1.3 and 2.2.1).

Example 1.2.4. In the Theory of Graphs $T_{\text {Graph }}$ (see Figure 1.1), for every $\ell \in \mathbb{N}$, let $K_{\ell}, P_{\ell} \in$ $\mathcal{M}_{\ell}$ denote respectively the complete graph on $\ell$ vertices and the path on $\ell$ vertices (i.e., of length $\ell-1)$. Furthermore, for every graph $G$, let $\bar{G}$ denote the complement of $G$.

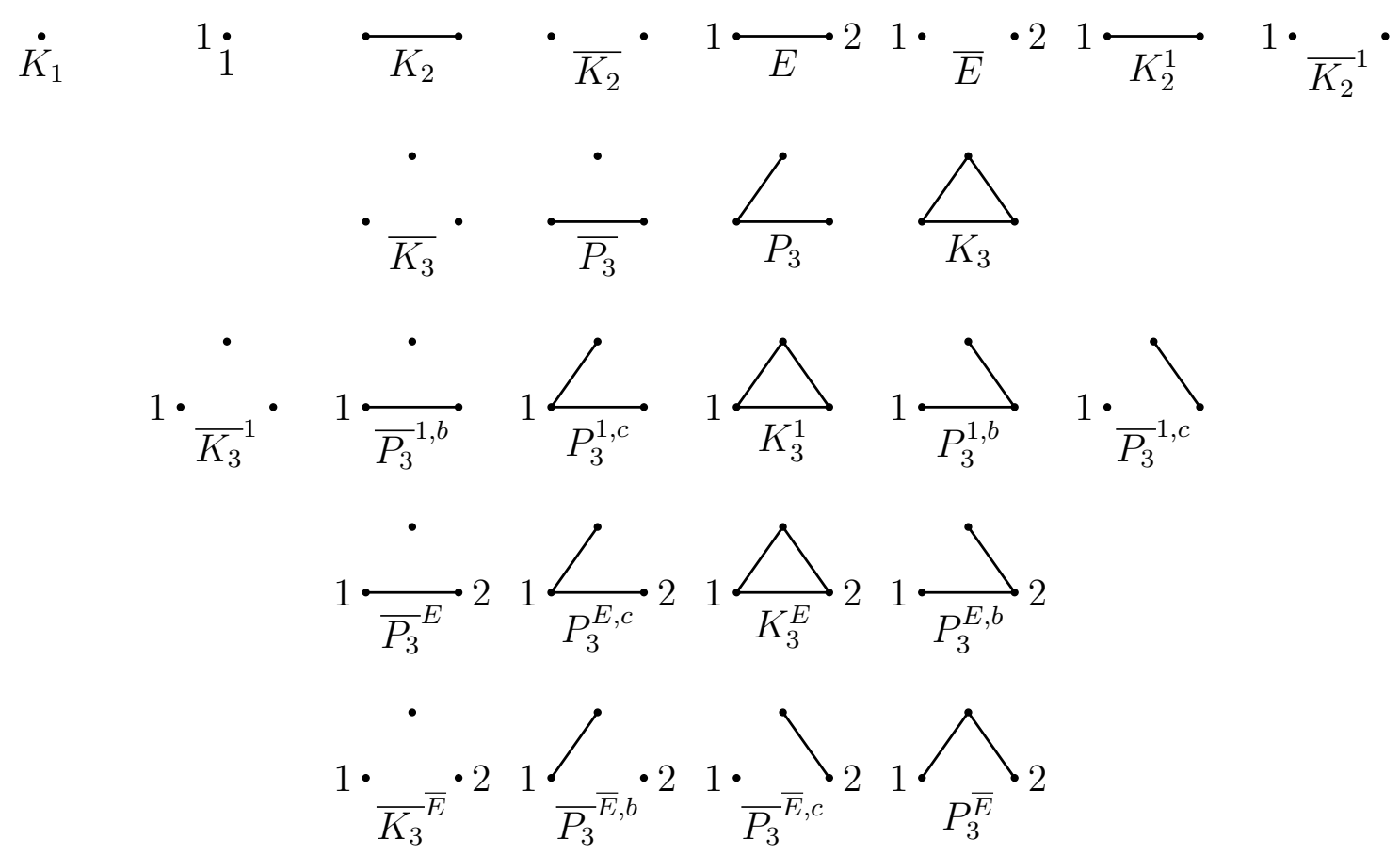

Figure 1.1: Models, types and flags in the Theory of graphs.

Let 1 denote the unique type of size 1 and let $E$ and $\bar{E}$ denote the types of size 2 corresponding respectively to the edge and the non-edge.

With our previously defined notation of $M^{\sigma}$ for a model $M$ and a type $\sigma$, we have already defined the 1-flags $K_{\ell}^{1}$ and $\bar{K}_{\ell}^{1}$, the E-flag $K_{\ell}^{E}$; and the $\bar{E}$-flag $\overline{K_{\ell}} \bar{E}$.

Note, however, that the notation $M^{\sigma}$ cannot be used for $P_{3}$ and $E$ neither for $\overline{P_{3}}$ and $\bar{E}$. For these cases, we will append $b$ or $c$ to the superscript to distinguish between the nonisomorphic flags.

Figure 1.1 includes definitions of more flags in $T_{\text {Graph }}$ and Table 1.1 includes several examples of joint densities in this theory.

Example 1.2.5. In the Theory of Permutations $T_{\text {Perm }}$, we denote a permutation $\tau \in \mathfrak{S}_{n}$ by listing its image as $(\tau(1) \tau(2) \cdots \tau(n))$. We also denote the unique type of size 1 in $T_{\text {Perm }}$ by 1. Finally, we denote a 1-flag in this theory by underlining the labelled vertex of the permutation, e.g., we denote by (312) the 1-flag over the model $\tau=(312)$ with label on vertex $\tau(3)=2$.

Tables 1.2 and 1.3 include several examples of joint densities in this theory. 


\begin{tabular}{c|cccc} 
& $\overline{K_{3}}$ & $\overline{P_{3}}$ & $P_{3}$ & $K_{3}$ \\
\hline$K_{1}$ & 1 & 1 & 1 & 1 \\
$K_{2}$ & 0 & $\frac{1}{3}$ & $\frac{2}{3}$ & 1 \\
$\overline{K_{2}}$ & 1 & $\frac{2}{3}$ & $\frac{1}{3}$ & 0
\end{tabular}

(a) Joint densities relative to type 0 .

\begin{tabular}{c|cccccc} 
& $\bar{K}_{3}^{1}$ & $\bar{P}_{3}^{1, b}$ & $P_{3}^{1, c}$ & $K_{3}^{1}$ & $P_{3}^{1, b}$ & $\bar{P}_{3}^{1, c}$ \\
\hline$K_{2}^{1}$ & 0 & $\frac{1}{2}$ & 1 & 1 & $\frac{1}{2}$ & 0 \\
$\bar{K}_{2}^{1}$ & 1 & $\frac{1}{2}$ & 0 & 0 & $\frac{1}{2}$ & 1 \\
$K_{2}^{1}, K_{2}^{1}$ & 0 & 0 & 1 & 1 & 0 & 0 \\
$K_{2}^{1}, \bar{K}_{2}^{1}$ & 0 & $\frac{1}{2}$ & 0 & 0 & $\frac{1}{2}$ & 0 \\
$\bar{K}_{2}^{1}, \bar{K}_{2}^{1}$ & 1 & 0 & 0 & 0 & 0 & 1
\end{tabular}

(b) Joint densities relative to type 1 .

Table 1.1: Joint densities in the Theory of graphs $p(A ; B)$, where $A$ is given by the leftmost entry of the line and $B$ by the topmost entry of the column.

\begin{tabular}{c|cccccc} 
& $(123)$ & $(132)$ & $(213)$ & $(231)$ & $(312)$ & $(321)$ \\
\hline$(1)$ & 1 & 1 & 1 & 1 & 1 & 1 \\
$(12)$ & 1 & $\frac{2}{3}$ & $\frac{2}{3}$ & $\frac{1}{3}$ & $\frac{1}{3}$ & 0 \\
$(21)$ & 0 & $\frac{1}{3}$ & $\frac{1}{3}$ & $\frac{2}{3}$ & $\frac{2}{3}$ & 1
\end{tabular}

Table 1.2: Joint densities $p(A ; B)$ in the Theory of permutations in type 0 , where $A$ is given by the leftmost entry of the line and $B$ by the topmost entry of the column. 


\begin{tabular}{|c|c|c|c|c|c|c|c|c|c|c|c|c|c|c|c|c|c|c|}
\hline & (123) & $(123)$ & $(12 \underline{3})$ & $(\underline{132})$ & $(1 \underline{3} 2)$ & $(13 \underline{2})$ & $(\underline{2} 13)$ & $(2 \underline{13})$ & $(21 \underline{3})$ & $(\underline{2} 31)$ & $(2 \underline{3} 1)$ & $(23 \underline{1})$ & $(\underline{3} 12)$ & $(3 \underline{12})$ & $(31 \underline{2})$ & $(\underline{3} 21)$ & $(321)$ & (321) \\
\hline$(12)$ & 1 & $\frac{1}{2}$ & 0 & 1 & 0 & 0 & $\frac{1}{2}$ & $\frac{1}{2}$ & 0 & $\frac{1}{2}$ & 0 & 0 & 0 & $\frac{1}{2}$ & 0 & 0 & 0 & 0 \\
\hline$(12 \underline{2})$ & 0 & $\frac{1}{2}$ & 1 & 0 & $\frac{1}{2}$ & $\frac{1}{2}$ & 0 & 0 & 1 & 0 & $\frac{1}{2}$ & 0 & 0 & 0 & $\frac{1}{2}$ & 0 & 0 & 0 \\
\hline$(\underline{2} 1)$ & 0 & 0 & 0 & 0 & $\frac{1}{2}$ & 0 & $\frac{1}{2}$ & 0 & 0 & $\frac{1}{2}$ & $\frac{1}{2}$ & 0 & 1 & 0 & 0 & 1 & $\frac{1}{2}$ & 0 \\
\hline$(2 \underline{1})$ & 0 & 0 & 0 & 0 & 0 & $\frac{1}{2}$ & 0 & $\frac{1}{2}$ & 0 & 0 & 0 & 1 & 0 & $\frac{1}{2}$ & $\frac{1}{2}$ & 0 & $\frac{1}{2}$ & 1 \\
\hline$(\underline{1} 2),(\underline{1} 2)$ & 1 & 0 & 0 & 1 & 0 & 0 & 0 & 0 & 0 & 0 & 0 & 0 & 0 & 0 & 0 & 0 & 0 & 0 \\
\hline$(1 \underline{2}),(1 \underline{2})$ & 0 & 0 & 1 & 0 & 0 & 0 & 0 & 0 & 1 & 0 & 0 & 0 & 0 & 0 & 0 & 0 & 0 & 0 \\
\hline$(\underline{2} 1),(\underline{2} 1)$ & 0 & 0 & 0 & 0 & 0 & 0 & 0 & 0 & 0 & 0 & 0 & 0 & 1 & 0 & 0 & 1 & 0 & 0 \\
\hline$(2 \underline{1}),(2 \underline{1})$ & 0 & 0 & 0 & 0 & 0 & 0 & 0 & 0 & 0 & 0 & 0 & 1 & 0 & 0 & 0 & 0 & 0 & 1 \\
\hline$(\underline{12}),(1 \underline{2})$ & 0 & $\frac{1}{2}$ & 0 & 0 & 0 & 0 & 0 & 0 & 0 & 0 & 0 & 0 & 0 & 0 & 0 & 0 & 0 & 0 \\
\hline$(\underline{1} 2),(\underline{2} 1)$ & 0 & 0 & 0 & 0 & 0 & 0 & $\frac{1}{2}$ & 0 & 0 & $\frac{1}{2}$ & 0 & 0 & 0 & 0 & 0 & 0 & 0 & 0 \\
\hline$(\underline{12}),(2 \underline{1})$ & 0 & 0 & 0 & 0 & 0 & 0 & 0 & $\frac{1}{2}$ & 0 & 0 & 0 & 0 & 0 & $\frac{1}{2}$ & 0 & 0 & 0 & 0 \\
\hline$(1 \underline{2}),(\underline{2} 1)$ & 0 & 0 & 0 & 0 & $\frac{1}{2}$ & 0 & 0 & 0 & 0 & 0 & $\frac{1}{2}$ & 0 & 0 & 0 & 0 & 0 & 0 & 0 \\
\hline$(1 \underline{2}),(2 \underline{1})$ & 0 & 0 & 0 & 0 & 0 & $\frac{1}{2}$ & 0 & 0 & 0 & 0 & 0 & 0 & 0 & 0 & $\frac{1}{2}$ & 0 & 0 & 0 \\
\hline$(\underline{2} 1),(2 \underline{1})$ & 0 & 0 & 0 & 0 & 0 & 0 & 0 & 0 & 0 & 0 & 0 & 0 & 0 & 0 & 0 & 0 & $\frac{1}{2}$ & 0 \\
\hline
\end{tabular}

Table 1.3: Joint densities $p(A ; B)$ in the Theory of permutations in type 1, where $A$ is given by the leftmost entry of the line and $B$ by the topmost entry of the column. 
The following two basic properties of joint density provide motivation for the flag algebra definitions to come.

Lemma 1.2.6 ([Raz07, Lemma 2.2]). Let $\sigma$ be a type of size $k$, let $\ell, \widetilde{\ell}, \ell_{1}, \ell_{2}, \ldots, \ell_{t} \geqslant k$ be integers such that

$$
\left(\sum_{i=1}^{t} \ell_{i}\right)-(t-1) k \leqslant \tilde{\ell} \leqslant \ell .
$$

and let $F, F_{1}, F_{2}, \ldots, F_{t} \in \mathcal{F}^{\sigma}$ be $\sigma$-flags of sizes $\ell, \ell_{1}, \ell_{2}, \ldots, \ell_{t}$ respectively.

Under these circumstances, we have

$$
p\left(F_{1}, F_{2}, \ldots, F_{t} ; F\right)=\sum_{\widetilde{F} \in \mathcal{F}_{\widetilde{\ell}}^{\sigma}} p\left(F_{1}, F_{2}, \ldots, F_{t} ; \widetilde{F}\right) p(\widetilde{F} ; F) .
$$

Lemma 1.2.7 ([Raz07, Lemma 2.3]). Under the same circumstances of Lemma 1.2.6, we have

$$
\left|p\left(F_{1}, F_{2}, \ldots, F_{t} ; F\right)-\prod_{i=1}^{t} p\left(F_{i} ; F\right)\right| \leqslant \frac{C}{\ell},
$$

for some constant $C$ that depends only on $\ell_{1}, \ell_{2}, \ldots, \ell_{t}$, but not on $\ell$.

Recall that we are interested in studying densities of small objects in large objects. This provides a motivation for the following definitions.

Definition 1.2.8 ([Raz07, Definition 7]). A sequence of $\sigma$-flags $\left(F_{n}\right)_{n \in \mathbb{N}}$ is increasing if $\left|F_{n}\right|<$ $\left|F_{n+1}\right|$ for every $n \in \mathbb{N}$.

A sequence of $\sigma$-flags $\left(F_{n}\right)_{n \in \mathbb{N}}$ is convergent if it is increasing and $\left(p\left(F ; F_{n}\right)\right)_{n \in \mathbb{N}}$ is convergent for every $\sigma$-flag $F \in \mathcal{F}^{\sigma}$.

Note that if $F \in \mathcal{F}^{\sigma}$ is a $\sigma$-flag then we can think of the functional $p(\cdot ; F)$ as an element $v$ of the compact metric space $[0,1]^{\mathcal{F}^{\sigma}}$, where the coordinate $F^{\prime}$ of $v$ is $p\left(F^{\prime} ; F\right)$, for every $F^{\prime} \in \mathcal{F}^{\sigma}$. The next lemma follows by compactness of $[0,1]^{\mathcal{F}^{\sigma}}$.

Lemma 1.2.9 ([Raz07, Theorem 3.2]). Every increasing sequence of $\sigma$-flags has a convergent subsequence.

Let us now provide some intuition of the definitions that will come. Suppose that $\left(F_{n}\right)_{n \in \mathbb{N}}$ is a convergent sequence of $\sigma$-flags and let $\phi: \mathcal{F}^{\sigma} \rightarrow \mathbb{R}$ be defined by $\phi(F)=\lim _{n \rightarrow \infty} p\left(F ; F_{n}\right)$.

Note that Lemma 1.2.6 with $t=1$ implies that, for every $F \in \mathcal{F}_{\ell}^{\sigma}$ of size $\ell$ and every $\tilde{\ell} \geqslant \ell$, we have

$$
\phi(F)=\sum_{\widetilde{F} \in \mathcal{F}_{\widetilde{\ell}}^{\sigma}} p(F ; \widetilde{F}) \phi(\widetilde{F}) .
$$

This suggests that we should think of $\phi$ not as a function with domain $\mathcal{F}^{\sigma}$, but rather with domain being a quotient of the set $\mathbb{R} \mathcal{F}^{\sigma}$ of formal linear combinations of $\sigma$-flags by relations given by Lemma 1.2.6.

Furthermore, if $F_{1}$ and $F_{2}$ are $\sigma$-flags of sizes $\ell_{1}$ and $\ell_{2}$ respectively and $\tilde{\ell} \geqslant \ell_{1}+\ell_{2}-|\sigma|$, then Lemma 1.2.6 with $t=2$ together with Lemma 1.2.7 says that

$$
\phi\left(F_{1}\right) \phi\left(F_{2}\right)=\sum_{\widetilde{F} \in \mathcal{F}_{\widetilde{\ell}}^{\sigma}} p\left(F_{1}, F_{2} ; \widetilde{F}\right) \phi(\widetilde{F}) .
$$


This provides intuition to define a product in the domain of the function $\phi$ that should be preserved by such functions obtained as limits of convergent sequences.

But before doing so, let us define the notion of a non-degenerate type.

Definition 1.2.10 ([Raz07, Definition 2]). A type $\sigma$ is non-degenerate if for every $\ell \geqslant|\sigma|$, we have $\mathcal{F}_{\ell}^{\sigma} \neq \varnothing$. Alternatively, the type is non-degenerate if $T^{\sigma}$ has an infinite model.

For completeness, we call a type $\sigma$ degenerate if it is not non-degenerate.

Proposition 1.2.11 ([Raz07, Lemma 2.4]). Let $\sigma$ be a non-degenerate type of size $k$ and let $\mathcal{A}^{\sigma}=\mathbb{R} \mathcal{F}^{\sigma} / \mathcal{K}^{\sigma}$ denote the quotient of the set $\mathbb{R} \mathcal{F}^{\sigma}$ of formal $\mathbb{R}$-linear combinations of elements of $\mathcal{F}^{\sigma}$ by the linear subspace $\mathcal{K}^{\sigma}$ generated by elements of the form

$$
F-\sum_{\widetilde{F} \in \mathcal{F}_{\widetilde{\ell}}^{\sigma}} p(F ; \widetilde{F}) \widetilde{F}
$$

where $\tilde{\ell} \geqslant|F|$.

Under these conditions, the product $\cdot: \mathcal{F}^{\sigma} \times \mathcal{F}^{\sigma} \rightarrow \mathcal{A}^{\sigma}$ defined by

$$
F_{1} \cdot F_{2}=\sum_{F \in \mathcal{F}_{\ell}^{\sigma}} p\left(F_{1}, F_{2} ; F\right) F
$$

for all flags $F_{1}, F_{2} \in \mathcal{F}^{\sigma}$ and every $\ell \geqslant\left|F_{1}\right|+\left|F_{2}\right|-k$ is well-defined.

Furthermore, the (bi)linear extension of this product to $\mathbb{R} \mathcal{F}^{\sigma} \times \mathbb{R} \mathcal{F}^{\sigma}$ induces a product in $\mathcal{A}^{\sigma}$, that is, for every $f \in \mathcal{A}^{\sigma}$ and every $g \in \mathcal{K}^{\sigma}$, we have $f \cdot g, g \cdot f \in \mathcal{K}^{\sigma}$.

Moreover, equipping $\mathcal{A}^{\sigma}$ with this product (and with the usual addition) makes it a commutative associative $\mathbb{R}$-algebra with unity $1_{\sigma}=(\sigma, \mathrm{Id})$ called $\sigma$-flag algebra.

In usual theories, all types are non-degenerate. We provide, however, the following straightforward lemma to remove non-degenerate types from any universal theory.

Lemma 1.2.12. If $T$ is a universal theory, then the theory of all models of $T$ that are not isomorphic to any degenerate type of $T$ can be formulated as a universal theory.

Proof. Take $\mathcal{F}$ as the family of all degenerate types and do as in Example 1.1.6.

One could think that by removing all non-degenerate types, we could end up with a theory without any model, but the following lemma says that this is not the case since $T$ has at least one infinite model.

Lemma 1.2.13. If $T$ has one infinite model $M$, then $M$ is a model of the theory $T^{\prime}$ obtained through Lemma 1.2.12.

Proof. It is enough to prove that there does not exist a degenerate type $\sigma$ that is a submodel of $M$. But this is trivially true, because if $W \subset V(M)$ is such that there exists an isomorphism $\theta$ between $\sigma$ and $\left.M\right|_{W}$, then for every $\ell \geqslant|\sigma|$, taking a set $X \subset V(M)$ with $|X|=\ell$ and $W \subset X$ yields $(X, \theta) \in \mathcal{F}_{\ell}^{\sigma}$, hence $\sigma$ is non-degenerate, a contradiction.

Let us finally provide a standard example of a degenerate type.

Example 1.2.14. Let $\sigma$ be a type of a theory $T$ of size $k$ and $\phi$ be the open diagram of $\sigma$. Let also $n>k$ be an integer and consider the theory $T^{\prime}$ obtained from $T$ by appending the axiom

$$
\forall x_{1} \forall x_{2} \cdots \forall x_{n}, \bigwedge_{i_{1}, i_{2}, \ldots, i_{k} \in[n]} \neg \phi\left(x_{i_{1}}, x_{i_{2}}, \ldots, x_{i_{k}}\right) .
$$


Since $n>k$, the type $\sigma$ is also a type of $T^{\prime}$. However, it is a degenerate type of $T^{\prime}$ since $\mathcal{F}_{m}^{\sigma}=\varnothing$ for every $m \geqslant n$.

Furthermore, if $M$ is an infinite model of $T$ that has no occurrence of $\sigma$, then $M$ is also an infinite model of $T$.

For instance, in the theory of graphs such that every four distinct vertices are triangle-free, the type corresponding to the triangle $K_{3}$ is degenerate.

Now that we have an $\mathbb{R}$-algebra, one of the most natural sets to study is the set of homomorphisms from it to the field $\mathbb{R}$.

Definition 1.2.15 ([Raz07, Definition 5]). Let $\sigma$ be a non-degenerate type. We denote the set of $\mathbb{R}$-algebra homomorphisms from $\mathcal{A}^{\sigma}$ to $\mathbb{R}$ by $\operatorname{Hom}\left(\mathcal{A}^{\sigma}, \mathbb{R}\right)$.

Furthermore, we define the set of positive homomorphisms from $\mathcal{A}^{\sigma}$ to $\mathbb{R}$ as the set

$$
\operatorname{Hom}^{+}\left(\mathcal{A}^{\sigma}, \mathbb{R}\right)=\left\{\phi \in \operatorname{Hom}\left(\mathcal{A}^{\sigma}, \mathbb{R}\right): \forall F \in \mathcal{F}^{\sigma}, \phi(F) \geqslant 0\right\} .
$$

Finally, let $\left(F_{n}\right)_{n \in \mathbb{N}}$ be a convergent sequence of flags and $\phi \in \operatorname{Hom}^{+}\left(\mathcal{A}^{\sigma}, \mathbb{R}\right)$. We say that $\left(F_{n}\right)_{n \in \mathbb{N}}$ converges to $\phi$ or that $\phi$ is the limit of the sequence $\left(F_{n}\right)_{n \in \mathbb{N}}$ if

$$
\lim _{n \rightarrow \infty} p\left(F ; F_{n}\right)=\phi(F)
$$

for every fixed $\sigma$-flag $F \in \mathcal{F}^{\sigma}$.

Remark 1.2.16. Since there will be no use for non-positive homomorphisms in this text, we will abuse terminology by calling a positive homomorphism just a homomorphism.

Remark 1.2.17. Note that if $\phi \in \operatorname{Hom}^{+}\left(\mathcal{A}^{\sigma}, \mathbb{R}\right)$, then we actually have $\phi(F) \in[0,1]$ for every $F \in \mathcal{F}^{\sigma}$.

This follows from $\phi\left(1_{\sigma}\right)=1$ and the fact that $\sum_{F \in \mathcal{F}_{\ell}^{\sigma}} F=1_{\sigma}$ for every $\ell \geqslant|\sigma|$.

From the observations we made previously, we know that any $\phi$ defined through $\phi(F)=$ $\lim _{n \rightarrow \infty} p\left(F ; F_{n}\right)$ for a convergent sequence $\left(F_{n}\right)_{n \in \mathbb{N}}$ must be an element of $\operatorname{Hom}^{+}\left(\mathcal{A}^{\sigma}, \mathbb{R}\right)$. The theorem below says that this set captures precisely the limits of convergent sequences.

Theorem 1.2.18 (Lovász-Szegedy [LS06], Razborov [Raz07, Theorem 3.3]). If $\sigma$ is a nondegenerate type, then every convergent sequence of $\sigma$-flags converges to a positive homomorphism and every positive homomorphism is the limit of a convergent sequence of $\sigma$-flags.

It will be useful to know a way of obtaining a sequence of flags that converges to a specific homomorphism $\phi$. Note first that since $\phi\left(1_{\sigma}\right)=1$, we have $\sum_{F \in \mathcal{F}_{\ell}^{\sigma}} \phi(F)=1$ for every $\ell \geqslant|\sigma|$.

Corollary 1.2.19 ([Raz07, Proof of Theorem 3.3]). Let $\phi \in \operatorname{Hom}^{+}\left(\mathcal{A}^{\sigma}, \mathbb{R}\right)$ be a homomorphism and, for every $n \in \mathbb{N}$, let $\boldsymbol{F}_{\boldsymbol{n}}$ be a random element of $\mathcal{F}_{n}^{\sigma}$ such that

$$
\mathbb{P}\left[\boldsymbol{F}_{\boldsymbol{n}} \cong F\right]=\phi(F)
$$

for every $F \in \mathcal{F}_{n}^{\sigma}$. Suppose furthermore that $\boldsymbol{F}_{\boldsymbol{n}}$ is independent of $\boldsymbol{F}_{\boldsymbol{m}}$ for $n \neq m$.

Under these circumstances, if $f: \mathbb{N} \rightarrow \mathbb{N}$ is a function such that $f(n)=\Omega\left(n^{2}\right)$, then the sequence $\left(\boldsymbol{F}_{\boldsymbol{f}(\boldsymbol{n})}\right)_{n \in \mathbb{N}}$ converges to $\phi$ almost surely.

\subsection{Homomorphism extensions}

In this section, we will present homomorphism extensions, which is a natural way of obtaining a random homomorphism $\phi^{\sigma_{\mathbf{2}}, \boldsymbol{\eta}} \in \operatorname{Hom}^{+}\left(\mathcal{A}^{\sigma_{2}}, \mathbb{R}\right)$ of the type $\sigma_{2}$ from a homomor- 
phism $\phi \in \operatorname{Hom}^{+}\left(\mathcal{A}^{\sigma_{1}}, \mathbb{R}\right)$ of the type $\sigma_{1}$, when $\sigma_{1}$ is "contained" in $\sigma_{2}$.

Before providing an intuition of what a homomorphism extension is, we must first define some notation.

Definition 1.3.1 ([Raz07, Section 2.2]). Let $\sigma$ be a type of size $k, k^{\prime} \leqslant k$ be a natural number and $\eta:\left[k^{\prime}\right] \rightarrow[k]$ be an injective function.

The type induced by $\eta$ in $\sigma$ is the unique type $\left.\sigma\right|_{\eta}$ such that $\eta$ is a model embedding of $\left.\sigma\right|_{\eta}$ in $\sigma$.

If $F=(M, \theta) \in \mathcal{F}^{\sigma}$ is a $\sigma$-flag, then we define the flag restriction $\left.F\right|_{\eta}$ as the flag $(M, \theta \circ \eta)$.

The intuition of a homomorphism extension is the following. Suppose that we have types $\sigma_{1}$ and $\sigma_{2}$ of sizes $k_{1}$ and $k_{2}$ respectively and $\eta:\left[k_{1}\right] \rightarrow\left[k_{2}\right]$ is an injective function such that $\left.\sigma_{2}\right|_{\eta}=\sigma_{1}$ and suppose that $\phi \in \operatorname{Hom}^{+}\left(\mathcal{A}^{\sigma_{1}}, \mathbb{R}\right)$ is a homomorphism such that $\phi\left(\left(\sigma_{2}, \eta\right)\right)>$ 0 . Theorem 1.2.18 says (intuitively) that $\phi$ represents an arbitrarily large $\sigma_{1}$-flag with $\left(\sigma_{2}, \eta\right)$ as a subflag. A natural and simple way of obtaining a large $\sigma_{2}$-flag (i.e., an element of $\left.\operatorname{Hom}^{+}\left(\mathcal{A}^{\sigma_{2}}, \mathbb{R}\right)\right)$ from $\phi$ would be to complete an embedding of $\sigma_{1}$ in $\phi$ to an embedding $\sigma_{2}$ uniformly at random, this is precisely the intuition behind the homomorphism extension $\phi^{\sigma_{2}, \eta}$. Before formalizing this, we must present some other definitions.

Definition 1.3.2 ([Raz07, Section 2.2]). A pair $\left(\sigma_{2}, \eta\right)$ is a type extension of a type $\sigma_{1}$ of size $k_{1}$ if $\sigma_{2}$ is a type and $\eta:\left[k_{1}\right] \rightarrow\left[\left|\sigma_{2}\right|\right]$ is such that $\left.\sigma_{2}\right|_{\eta}=\sigma_{1}$ (note that we require equality instead of isomorphism).

If $\left(\sigma_{2}, \eta\right)$ is a type extension of $\sigma_{1}$ and $F=(M, \theta) \in \mathcal{F}^{\sigma_{2}}$ is a $\sigma_{2}$-flag, we define the normalizing factor $q_{\sigma_{2}, \eta}$ through the following random experiment. Pick uniformly at random an injective function $\boldsymbol{\alpha}:\left[k_{2}\right] \rightarrow V(M)$ subject to the restriction that $\boldsymbol{\alpha}$ is consistent with $\theta$ on $\operatorname{im}(\eta)$, that is, subject to $\boldsymbol{\alpha} \circ \eta=\theta \circ \eta$, and define

$$
q_{\sigma_{2}, \eta}(F)=\mathbb{P}\left[\boldsymbol{\alpha} \text { is a model embedding of } \sigma_{2} \text { in } M\right] .
$$

Lemma 1.3.3 ([Raz07, Theorem 2.5]). If $\left(\sigma_{2}, \eta\right)$ is a type extension of a non-degenerate type $\sigma_{1}$, then the downward operator $\llbracket \cdot \rrbracket_{\sigma_{2}, \eta}$ defined by letting

$$
\llbracket F \rrbracket_{\sigma_{2}, \eta}=\left.q_{\sigma_{2}, \eta}(F) F\right|_{\eta},
$$

for every $\sigma_{2}$-flag $F \in \mathcal{F}^{\sigma_{2}}$ and extending it linearly to $\mathbb{R} \mathcal{F}^{\sigma_{2}}$ induces a well-defined linear operator from $\mathcal{A}^{\sigma_{2}}$ to $\mathcal{A}^{\sigma_{1}}$, that is, we have $\llbracket \mathcal{K}^{\sigma_{2}} \rrbracket_{\sigma_{2}, \eta} \subset \mathcal{K}^{\sigma_{1}}$.

Example 1.3.4. In the Theory of Graphs, our notation makes computing the downward operator easy when the smaller type is 0. See Tables 1.4, 1.5 and 1.6 for some examples in this theory.

\begin{tabular}{l|ccccccccc} 
& $1_{1}$ & $K_{2}^{1}$ & $\bar{K}_{2}^{1}$ & $\bar{K}_{3}^{1}$ & $\bar{P}_{3}^{1, b}$ & $P_{3}^{1, c}$ & $K_{3}^{1}$ & $P_{3}^{1, b}$ & $\bar{P}_{3}^{1, c}$ \\
\hline$\llbracket \cdot \rrbracket_{1}$ & $K_{1}$ & $K_{2}$ & $\overline{K_{2}}$ & $\overline{K_{3}}$ & $\frac{2}{3} \overline{P_{3}}$ & $\frac{1}{3} P_{3}$ & $K_{3}$ & $\frac{2}{3} P_{3}$ & $\frac{1}{3} \overline{P_{3}}$
\end{tabular}

Table 1.4: Downward operator in the Theory of graphs from type 1 to type 0 (the function $\eta$ is omitted from the notation).

Example 1.3.5. In the Theory of Permutations, it is easy to see that if $\left(\sigma_{2}, \eta\right)$ is a type 


\begin{tabular}{c|ccccc} 
& $1_{E}$ & $\bar{P}_{3}^{E}$ & $P_{3}^{E, c}$ & $K_{3}^{E}$ & $P_{3}^{E, b}$ \\
\hline$\llbracket \cdot \rrbracket_{E, \eta_{1}}$ & $K_{2}^{1}$ & $\frac{1}{2} \bar{P}_{3}^{1, b}$ & $P_{3}^{1, c}$ & $K_{3}^{1}$ & $\frac{1}{2} P_{3}^{1, b}$ \\
$\llbracket \cdot \rrbracket_{E, \eta_{2}}$ & $K_{2}^{1}$ & $\frac{1}{2} \bar{P}_{3}^{1, b}$ & $\frac{1}{2} P_{3}^{1, b}$ & $K_{3}^{1}$ & $P_{3}^{1, c}$ \\
$\llbracket \cdot \rrbracket_{E}$ & $K_{2}$ & $\frac{1}{3} \overline{P_{3}}$ & $\frac{1}{3} P_{3}$ & $K_{3}$ & $\frac{1}{3} P_{3}$
\end{tabular}

Table 1.5: Downward operator in the Theory of graphs from type $E$ to types 1 and 0 . The function $\eta_{i}:[1] \rightarrow[2]$ is such that $\eta(1)=i$ and the function $\eta$ is omitted from the notation for type 0 .

\begin{tabular}{c|ccccc} 
& $1_{\bar{E}}$ & $\overline{K_{3}}$ & $\overline{P_{3}}{ }^{\bar{E}}, b$ & $\bar{P}_{3}^{\bar{E}, c}$ & $P_{3}^{\bar{E}}$ \\
\hline$\llbracket \cdot \rrbracket_{\bar{E}, \eta_{1}}$ & $\bar{K}_{2}^{1}$ & $\overline{K_{3}}$ & $\overline{1}_{\bar{P}_{3}}^{1, b}$ & ${\overline{P_{3}}}^{1, c}$ & $\frac{1}{2} P_{3}^{1, b}$ \\
$\llbracket \cdot \rrbracket_{\bar{E}, \eta_{2}}$ & $\overline{K_{2}}$ & $\overline{K_{3}}$ & ${\overline{P_{3}}}^{1, c}$ & $\frac{1}{2}{\overline{P_{3}}}^{1, b}$ & $\frac{1}{2} P_{3}^{1, b}$ \\
$\llbracket \cdot \rrbracket_{\bar{E}}$ & $\overline{K_{2}}$ & $\overline{K_{3}}$ & $\frac{1}{3} \overline{P_{3}}$ & $\frac{1}{3} \overline{P_{3}}$ & $\frac{1}{3} P_{3}$
\end{tabular}

Table 1.6: Downward operator in the Theory of graphs from type $\bar{E}$ to types 1 and 0 . The function $\eta_{i}:[1] \rightarrow[2]$ is such that $\eta(1)=i$ and the function $\eta$ is omitted from the notation for type 0 .

extension of $\sigma_{1}$, with $\left|\sigma_{i}\right|=k_{i}$ for $i=1,2$, and if $F \in \mathcal{F}_{\ell}^{\sigma_{2}}$ is a $\sigma_{2}$-flag of size $\ell$, then

$$
q_{\sigma_{2}, \eta}(F)=\frac{1}{(\ell)_{k_{2}-k_{1}}}=\frac{1}{\ell(\ell-1) \cdots\left(\ell-k_{2}+k_{1}+1\right)} .
$$

The next step is to define the finite world analogous of the homomorphism extension, which will be useful in obtaining the extension itself.

Before doing so, recall that if $F \in \mathcal{F}^{\sigma}$ is a $\sigma$-flag, we can think of the functional $p(\cdot ; F)$ as an element of the compact metric space $[0,1]^{\mathcal{F}^{\sigma}}$. On the other hand, we can also think of a homomorphism $\phi \in \operatorname{Hom}^{+}\left(\mathcal{A}^{\sigma}, \mathbb{R}\right)$ as an element $w$ of $[0,1]^{\mathcal{F}^{\sigma}}$, where the coordinate $F^{\prime}$ of $w$ is $\phi\left(F^{\prime}\right)$, for every $F^{\prime} \in \mathcal{F}^{\sigma}$.

Definition 1.3.6 ([Raz07, Definition 9]). Let $\left(\sigma_{2}, \eta\right)$ be a type extension of $\sigma_{1}$ and $F=$ $(M, \theta) \in \mathcal{F}^{\sigma_{1}}$-flag such that $p\left(\left(\sigma_{2}, \eta\right) ; F\right)>0$. We define the probability measure $\mathbb{P}_{F}^{\sigma_{2}, \eta}$ extending $F$ to $\sigma_{2}$ through $\eta$ through the following random experiment. Pick uniformly at random a model embedding $\boldsymbol{\alpha}:\left[\left|\sigma_{2}\right|\right] \rightarrow V(M)$ of $\sigma_{2}$ in $M$ subject to the restriction that $\boldsymbol{\alpha}$ is consistent with $\theta$ on $\operatorname{im}(\eta)$, that is, subject to $\boldsymbol{\alpha} \circ \eta=\theta \circ \eta$, and define $\mathbb{P}_{F}^{\sigma_{2}, \eta}$ as the (discrete) Borel probability measure over $[0,1]^{\mathcal{F}_{2}}$ of the functional $p(\cdot ;(M, \boldsymbol{\alpha}))$.

The main theorem of this section says that the limit world analogous of this operation (which are the homomorphism extensions) can be obtained as a the weak limit of the finite world operation. To make this precise we recall the notion of weak convergence of probability measures below. 
Definition 1.3.7. Let $X$ be a topological space and $\left(\mathbb{P}_{n}\right)_{n \in \mathbb{N}}$ be a sequence of Borel probability measures on $X$.

We say that $\left(\mathbb{P}_{n}\right)_{n \in \mathbb{N}}$ weakly converges to a Borel probability measure $\mathbb{P}$ on $X$ if

$$
\lim _{n \rightarrow \infty} \int_{X} f(x) d \mathbb{P}_{n}(x)=\int_{X} f(x) d \mathbb{P}(x)
$$

for every continuous bounded function $f: X \rightarrow \mathbb{R}$.

Remark 1.3.8. In the case when $X$ is a metric space, the definition of weak convergence coincides with the definition of convergence in distribution.

We recall now one of the most useful equivalences concerning weak convergence.

Lemma 1.3.9 (Portmanteau). If $X$ is a topological space, $\left(\mathbb{P}_{n}\right)_{n \in \mathbb{N}}$ is a sequence of Borel probability measures on $X$, and $\mathbb{P}$ is a Borel probability measure on $X$, then the following are equivalent.

- The sequence $\left(\mathbb{P}_{n}\right)_{n \in \mathbb{N}}$ weakly converges to $\mathbb{P}$;

- For every Borel set $A \in \mathcal{B}(X)$ of $X$ with $\mathbb{P}(\delta(A))=0$ (where $\delta(A)$ is the boundary of $A$ ), we have

$$
\lim _{n \rightarrow \infty} \mathbb{P}_{n}(A)=\mathbb{P}(A)
$$

- For every open set $U \subset X$, we have

$$
\liminf _{n \rightarrow \infty} \mathbb{P}_{n}(U) \geqslant \mathbb{P}(U)
$$

- For every closed set $C \subset X$, we have

$$
\limsup _{n \rightarrow \infty} \mathbb{P}_{n}(C) \leqslant \mathbb{P}(C)
$$

We present (finally) the homomorphism extensions in the theorem below.

Theorem 1.3.10 ([Raz07, Theorems 3.5 and 3.12]). Let $\left(\sigma_{2}, \eta\right)$ be a type extension of a nondegenerate type $\sigma_{1}$ and let $\phi \in \operatorname{Hom}^{+}\left(\mathcal{A}^{\sigma_{1}}, \mathbb{R}\right)$ be a homomorphism such that $\phi\left(\left(\sigma_{2}, \eta\right)\right)>0$.

Under these circumstances, there exists a random element $\phi^{\sigma_{2}, \boldsymbol{\eta}}$ of $\operatorname{Hom}^{+}\left(\mathcal{A}^{\sigma_{2}}, \mathbb{R}\right)$, called homomorphism extension of $\phi$ to type $\sigma_{2}$ through $\eta$, satisfying

$$
\mathbb{E}\left[\phi^{\sigma_{2}, \eta}(f)\right]=\frac{\phi\left(\llbracket f \rrbracket_{\sigma_{2}, \eta}\right)}{\phi\left(\llbracket 1_{\sigma_{2}} \rrbracket_{\sigma_{2}, \eta}\right)},
$$

for every $f \in \mathcal{A}^{\sigma_{2}}$.

Furthermore, the distribution of $\phi^{\sigma_{2}, \eta}$ is unique, that is, if $\mathbb{P}^{\sigma_{2}, \eta}$ is the probability measure of $\boldsymbol{\phi}^{\boldsymbol{\sigma}_{\mathbf{2}}, \boldsymbol{\eta}}$, and $\mathbb{P}^{\prime}$ is another probability measure on the Borel sets of $\operatorname{Hom}^{+}\left(\mathcal{A}^{\sigma_{2}}, \mathbb{R}\right)$ satisfying (1.2), then $\mathbb{P}^{\sigma_{2}, \eta}=\mathbb{P}^{\prime}$.

Moreover, if $\left(F_{n}\right)_{n \in \mathbb{N}}$ is a sequence of $\sigma_{1}$-flags converging to $\phi$, then the sequence of probability measures $\left(\mathbb{P}_{F_{n}}^{\sigma_{2}, \eta}\right)_{n \in \mathbb{N}}$ weakly converges to the probability measure of the extension $\boldsymbol{\phi}^{\boldsymbol{\sigma}_{\mathbf{2}}, \boldsymbol{\eta}}$ (as a random element of $[0,1]^{\mathcal{F}^{\sigma_{2}}}$ ).

Remark 1.3.11. As a final (but important) remark of this section, when the smaller type is 0 , we will use notations that either replace $\eta$ by 0 or completely omit it, e.g., we will use $\left.F\right|_{0}, q_{\sigma}, \llbracket \cdot \rrbracket_{0}, \mathbb{P}_{F}^{\sigma}$ and $\phi^{\sigma}$. 


\subsection{Interpretation homomorphisms}

In this section, we will present the interpretation homomorphisms, which are algebra homomorphisms between flag algebras of different types (and even in different theories) that will codify classical combinatorial arguments of many sorts. We choose to first present the definitions and properties of the interpretation homomorphisms leaving the intuition and examples to the end of the section ${ }^{2}$.

We start with the definition of flag interpretation, which corresponds to a model interpretation followed by the removal of constants that have are not translations of other constants (see Appendix A for the concepts of open interpretation and model interpretation).

Definition 1.4.1. Let $T_{1}$ and $T_{2}$ be two universal theories in languages with equality, but no constant or function symbols. Let $\sigma_{1}$ and $\sigma_{2}$ be non-degenerate types in $T_{1}$ and $T_{2}$ respectively, and of sizes $k_{1}$ and $k_{2}$ respectively.

Recall the definitions of $T_{i}^{\sigma_{i}}$ and of $\mathcal{F}^{\sigma_{i}}\left[T_{i}\right]$ and suppose $(U, I): T_{1}^{\sigma_{1}} \rightsquigarrow T_{2}^{\sigma_{2}}$ is an open interpretation, let $c_{1}, c_{2}, \ldots, c_{k_{1}}$ be the constant symbols of $T_{1}^{\sigma_{1}}$ and $c_{1}^{\prime}, c_{2}^{\prime}, \ldots, c_{k_{2}}^{\prime}$ be the constant symbols of $T_{2}^{\sigma_{2}}$ and define the function $\eta:\left[k_{1}\right] \rightarrow\left[k_{2}\right]$ to be such that $I\left(c_{i}\right)=c_{\eta(i)}^{\prime}$ for every $i \in\left[k_{1}\right]$.

Note that $I$ applied to the formula $\bigwedge_{i, j=1}^{k_{1}}\left(i=j \Longleftrightarrow c_{i}=c_{j}\right)$ implies that $\eta$ is injective.

Let $\mathcal{F}^{\sigma_{2}, U}\left[T_{2}\right]$ denote the set of all $\sigma_{2}$-flags that are $U$-models of $T_{2}^{\sigma_{2}}$ and for every $\ell \in \mathbb{N}$, let $\mathcal{F}_{\ell}^{\sigma_{2}, U}\left[T_{2}\right]=\mathcal{F}^{\sigma_{2}, U}\left[T_{2}\right] \cap \mathcal{F}_{\ell}^{\sigma_{2}}\left[T_{2}\right]$.

Suppose $F=(M, \theta) \in \mathcal{F}^{\sigma_{2}, U}\left[T_{2}\right]$ is a $U$-model. Then the model interpretation of $F$ is a flag $I(F)=(N, \theta \circ \eta)$ of $T_{1}$ with $V(N)=V(M)$.

We define then the flag interpretation of $F$ as the $\sigma_{1}$-flag

$$
I^{\prime}(F)=I(F)-\theta\left(\left[k_{2}\right] \backslash \eta\left(\left[k_{1}\right]\right)\right)=\left.I(F)\right|_{V(I(F)) \backslash \theta\left(\left[k_{2}\right] \backslash \eta\left(\left[k_{1}\right]\right)\right)} .
$$

Remark 1.4.2. The only case when a flag interpretation coincides with the model interpretation is when both types are of the same size.

Theorem 1.4.3 ([Raz07, Theorem 2.6]). With the same definitions and notation of Definition 1.4.1, let

$$
u=\sum_{F \in \mathcal{F}_{k_{2}+1}^{\sigma_{2}, U}\left[T_{2}\right]} F
$$

be the sum of all $U$-models of size $k_{2}+1$ (that is, the $U$-models that have exactly one unlabelled vertex) and suppose that $u$ is not a zero divisor in $\mathcal{A}^{\sigma_{2}}\left[T_{2}\right]$.

Let $\mathcal{A}_{u}^{\sigma_{2}}\left[T_{2}\right]$ denote the localization of the algebra $\mathcal{A}^{\sigma_{2}}\left[T_{1}\right]$ with respect to the multiplicative system $\left\{u^{\ell}: \ell \in \mathbb{N}\right\}$ (that is, every element of $\mathcal{A}_{u}^{\sigma_{2}}\left[T_{2}\right]$ is of the form $u^{-\ell} f$ for $\ell \in \mathbb{N}$ and $\left.f \in \mathcal{A}^{\sigma_{2}}\left[T_{2}\right]\right)$.

For every $\sigma_{1}$-flag $F_{1} \in \mathcal{F}_{\ell_{1}}^{\sigma_{1}}\left[T_{1}\right]$ of size $\ell_{1}$, define

$$
\pi^{(U, I)}\left(F_{1}\right)=\frac{1}{u^{\ell_{1}-k_{1}}} \sum_{\substack{F_{2} \in \mathcal{F}^{\sigma_{2}, U}\left[T_{2}\right]: \\ I^{\prime}\left(F_{2}\right) \cong F_{1}}} F_{2} \quad \in \mathcal{A}_{u}^{\sigma_{2}}\left[T_{2}\right],
$$

and extend the operator $\pi^{(U, I)}$ linearly to $\mathbb{R} \mathcal{F}^{\sigma_{1}}\left[T_{1}\right]$ (note that $I^{\prime}\left(F_{2}\right) \cong F_{1}$ implies $\left|F_{2}\right|=$ $\left.\ell_{1}-k_{1}+k_{2}\right)$.

\footnotetext{
${ }^{2}$ The reader may want to skip to this final part whenever the definitions and properties start to look too abstract.
} 
Under these circumstances, we have $\pi^{(U, I)}\left(\mathcal{K}^{\sigma_{1}}\left[T_{1}\right]\right)=\{0\}$ and the induced linear operator

$$
\pi^{(U, I)}: \mathcal{A}^{\sigma_{1}}\left[T_{1}\right] \longrightarrow \mathcal{A}_{u}^{\sigma_{2}}\left[T_{2}\right]
$$

is an algebra homomorphism, called interpretation homomorphism.

In the next three subsections, we will present three special cases of interpretation homomorphisms concerning special $U$ and $I$ providing the intuition for each case at the end of the subsection $^{3}$. The setup of the next subsections will always be that of Theorem 1.4.3.

\subsubsection{Algebras of constants: upward operator}

Consider the case when $T_{1}=T_{2}$ (and let us denote this theory by simply $T$ in this subsection and drop it from notation), when the translation $I$ fixes predicate symbols, and when $U \equiv \top$ is identically true. Note that in this case we have that $\left(\sigma_{2}, \eta\right)$ is a type extension of $\sigma_{1}$ and $u=1_{\sigma_{2}}$ so the localization $\mathcal{A}_{u}^{\sigma_{2}}$ coincides with the flag algebra $\mathcal{A}^{\sigma_{2}}$.

In this special case, the interpretation homomorphism $\pi^{(U, I)}$ is called upward operator and is denoted $\pi^{\sigma_{2}, \eta}$.

Furthermore, we also give a special name to the flag interpretation of this special case. For a $\sigma_{1}$-flag $F=(M, \theta) \in \mathcal{F}^{\sigma_{1}}$, the flag removing restriction of $F$, denoted by $F \downarrow_{\eta}$, is defined as

$$
F \downarrow_{\eta}=I^{\prime}(F)=\left.F\right|_{\eta}-\theta\left(\left[k_{2}\right] \backslash \eta\left(\left[k_{1}\right]\right)\right)=\left.\left(\left.F\right|_{\eta}\right)\right|_{V(M) \backslash \theta\left(\left[k_{2}\right] \backslash \eta\left(\left[k_{1}\right]\right)\right)} .
$$

With this notation, the upward operator is an algebra homomorphism $\pi^{\sigma_{2}, \eta}: \mathcal{A}^{\sigma_{1}} \rightarrow \mathcal{A}^{\sigma_{2}}$ and can be computed through

$$
\pi^{\sigma_{2}, \eta}(F)=\sum_{\substack{\widetilde{F} \in \mathcal{F}^{\sigma_{2}}: \\ \widetilde{F} \downarrow_{\eta} \cong F}} \widetilde{F}
$$

(Again, we have that $\widetilde{F} \downarrow_{\eta} \cong F$ implies $|\widetilde{F}|=|F|-k_{1}+k_{2}$.)

Following Remark 1.3.11, when $\sigma_{1}=0$, we will use the notation $\pi^{\sigma_{2}}$ and $F \downarrow_{0}$.

The intuition behind upward operators will come from the next theorem and its corollary.

Theorem 1.4.4 ([Raz07, Theorem 2.8a]). Let $\left(\sigma_{2}, \eta\right)$ be a type extension of a non-degenerate type $\sigma_{1}$. If $f \in \mathcal{A}^{\sigma_{1}}$ and $g \in \mathcal{A}^{\sigma_{2}}$, then

$$
\llbracket \pi^{\sigma_{2}, \eta}(f) g \rrbracket_{\sigma_{2}, \eta}=f \llbracket g \rrbracket_{\sigma_{2}, \eta} .
$$

In particular, we have

$$
\llbracket \pi^{\sigma_{2}, \eta}(f) \rrbracket_{\sigma_{2}, \eta}=f \llbracket 1_{\sigma_{2}} \rrbracket_{\sigma_{2}, \eta} .
$$

Corollary 1.4.5 ([Raz07, Corollary 3.19]). If $\left(\sigma_{2}, \eta\right)$ is a type extension of a non-degenerate type $\sigma_{1}$ and $\phi \in \operatorname{Hom}^{+}\left(\mathcal{A}^{\sigma_{1}}, \mathbb{R}\right)$ is such that $\phi\left(\left(\sigma_{2}, \eta\right)\right)>0$, then

$$
\phi^{\sigma_{2}, \eta} \circ \pi^{\sigma_{2}, \eta}=\phi \text { a.s. }
$$

Remark 1.4.6. This corollary also justifies the name "algebras of constants" given to this subsection: the upward operator $\pi^{\sigma_{2}, \eta}$ gives elements of $\mathcal{A}^{\sigma_{2}}$ that are constants for the

\footnotetext{
${ }^{3}$ The reader can then reason about the intuition of the general case, which will be a composition of all cases.
} 
extension $\phi^{\sigma_{2}, \eta}$.

Let us now present the intuition of this corollary and of the upward operators. For simplicity, let us suppose that $\eta(i)=i$ for every $i \in\left[k_{1}\right]$.

Suppose we want to compute the density of a $\sigma_{1}$-flag $F$ in a large $\sigma_{1}$-flag represented by the homomorphism $\phi$. We already know that this density is $\phi\left(F_{1}\right)$, but we may compute it through a different method.

First we pick uniformly at random an occurrence of $\sigma_{2}$ extending $\sigma_{1}$ in the large flag; this yields the extension $\boldsymbol{\phi}^{\boldsymbol{\sigma}_{2}, \boldsymbol{\eta}}$. Then we compute the density of a " $\sigma_{2}$-flag" $F_{2}=(M, \theta)$ in which $V\left(F_{2}\right) \backslash \theta\left(\left[k_{2}\right] \backslash \eta\left(\left[k_{1}\right]\right)\right)$ is an occurrence of $F_{1}$ in $F_{2}$; this " $\sigma_{2}$-flag" is the $\sigma_{2}$-flag algebra element $\pi^{\sigma_{2}, \eta}\left(F_{1}\right)$, and the sought density is $\boldsymbol{\phi}^{\sigma_{2}, \eta} \circ \pi^{\sigma_{2}, \eta}\left(F_{1}\right)$.

Intuitively, it is clear that the second method also yields the density of $F_{1}$ in $\phi$, that is, we have

$$
\boldsymbol{\phi}^{\boldsymbol{\sigma}_{2}, \boldsymbol{\eta}} \circ \pi^{\sigma_{2}, \eta}\left(F_{1}\right)=\phi\left(F_{1}\right) \text { a.s. }
$$

as says Theorem 1.4.4. This also says that the element $\pi^{\sigma_{2}, \eta}\left(F_{1}\right)$ is the $\sigma_{2}$-flag algebra equivalent of the $\sigma_{1}$-flag $F_{1}$.

The next theorem takes this "equivalence" one step further by considering the case when initial homomorphism is already a homomorphism extension.

Theorem 1.4.7 ([Raz07, Theorem 3.17]). Let $\left(\sigma_{2}, \eta\right)$ be a type extension of a non-degenerate type $\sigma_{1}$ and let $\phi \in \operatorname{Hom}^{+}\left(\mathcal{A}^{\sigma_{1}}, \mathbb{R}\right)$ be a homomorphism such that $\phi\left(\left(\sigma_{2}, \eta\right)\right)>0$. Let also $k_{1}=\left|\sigma_{1}\right|, k_{2}=\left|\sigma_{2}\right|, k_{3} \geqslant k_{2}$ be an integer and $\nu:\left[k_{2}\right] \rightarrow\left[k_{3}\right]$ be an injective function.

Let $\operatorname{Ext}\left(\sigma_{2}, \nu\right)$ denote the set of all types $\sigma_{3}$ such that $\left(\sigma_{3}, \nu\right)$ is a type extension of $\sigma_{2}$ (this forces $\left|\sigma_{3}\right|=k_{3}$ ).

Under these circumstances, we have

$$
\sum_{\sigma_{3} \in \operatorname{Ext}\left(\sigma_{2}, \nu\right)} \phi\left(\llbracket 1_{\sigma_{3}} \rrbracket_{\sigma_{3}, \nu \circ \eta}\right)=\phi\left(\llbracket 1_{\sigma_{2}} \rrbracket_{\sigma_{2}, \eta}\right) .
$$

Furthermore, if $\boldsymbol{\sigma}_{\mathbf{3}} \in \operatorname{Ext}\left(\sigma_{3}, \nu\right)$ is a random type in $\operatorname{Ext}\left(\sigma_{2}, \nu\right)$ such that

$$
\mathbb{P}\left[\boldsymbol{\sigma}_{3}=\widetilde{\sigma_{3}}\right]=\frac{\phi\left(\llbracket 1_{\widetilde{\sigma_{3}}} \rrbracket_{\widetilde{\sigma_{3}}, \nu \circ \eta}\right)}{\phi\left(\llbracket 1_{\sigma_{2}} \rrbracket_{\sigma_{2}, \eta}\right)},
$$

then the random homomorphism

$$
\phi^{\sigma_{3}, \nu \circ \eta} \circ \pi^{\sigma_{3}, \nu} \in \operatorname{Hom}^{+}\left(\mathcal{A}^{\sigma_{2}}, \mathbb{R}\right)
$$

has the same distribution as the extension $\phi^{\sigma_{2}, \eta}$.

Finally, we present examples involving $\pi^{\sigma_{2}, \eta}$.

Example 1.4.8. For $i=1,2$, let $\eta_{i}:[1] \rightarrow[2]$ denote the function such that $\eta(1)=i$. In the 
Theory of Graphs, we have

$$
\begin{aligned}
& \pi^{1}\left(K_{1}\right)=K_{2}^{1}+{\overline{K_{2}}}^{1} ; \quad \pi^{1}\left(K_{2}\right)=K_{3}^{1}+P_{3}^{1, b}+{\overline{P_{3}}}^{1, c} ; \quad \pi^{1}\left(\overline{K_{2}}\right)={\overline{K_{3}}}^{1}+{\overline{P_{3}}}^{1, b}+P_{3}^{1, c} ; \\
& \pi^{E, \eta_{1}}\left(K_{1}^{1}\right)=1_{E} ; \quad \pi^{E, \eta_{1}}\left(K_{2}^{1}\right)=P_{3}^{E, c}+K_{3}^{E} ; \quad \pi^{E, \eta_{1}}\left({\overline{K_{2}}}^{1}\right)={\overline{P_{3}}}^{E}+P_{3}^{E, b} ; \\
& \pi^{E, \eta_{2}}\left(K_{1}^{1}\right)=1_{E} ; \quad \pi^{E, \eta_{2}}\left(K_{2}^{1}\right)=K_{3}^{E}+P_{3}^{E, b} ; \quad \pi^{E, \eta_{2}}\left({\overline{K_{2}}}^{1}\right)={\overline{P_{3}}}^{E}+P_{3}^{E, c} ; \\
& \pi^{\bar{E}, \eta_{1}}\left(K_{1}^{1}\right)=1_{\bar{E}} ; \quad \pi^{\bar{E}, \eta_{1}}\left(K_{2}^{1}\right)={\overline{P_{3}}}^{\bar{E}, b}+P_{3}^{\bar{E}} ; \quad \pi^{\bar{E}, \eta_{1}}\left({\overline{K_{2}}}^{1}\right)=\bar{K}^{\bar{E}}+{\overline{P_{3}}}^{\bar{E}, c} ; \\
& \pi^{\bar{E}, \eta_{2}}\left(K_{1}^{1}\right)=1_{\bar{E}} ; \quad \pi^{\bar{E}, \eta_{2}}\left(K_{2}^{1}\right)={\overline{P_{3}}}^{\bar{E}, c}+P_{3}^{\bar{E}} ; \quad \pi^{\bar{E}, \eta_{2}}\left({\overline{K_{2}}}^{1}\right)={\overline{K_{3}}}^{\bar{E}}+{\overline{P_{3}}}^{\bar{E}, b} \text {. }
\end{aligned}
$$

Example 1.4.9. In the Theory of Permutations, we have

$$
\begin{aligned}
\pi^{1}((1)) & =(\underline{1} 2)+(\underline{2} 1)+(1 \underline{2})+(2 \underline{1}) ; \\
\pi^{1}((12)) & =(\underline{1} 23)+(1 \underline{2} 3)+(12 \underline{3})+(1 \underline{3} 2)+(13 \underline{2})+(\underline{2} 13)+(2 \underline{1} 3)+(23 \underline{1})+(\underline{3} 12) ; \\
\pi^{1}((21)) & =(\underline{1} 32)+(21 \underline{3})+(\underline{2} 31)+(2 \underline{3} 1)+(3 \underline{12})+(31 \underline{2})+(\underline{3} 21)+(3 \underline{2} 1)+(32 \underline{1}) .
\end{aligned}
$$

\subsubsection{Inductive arguments}

We consider now the case when $T_{1}=T_{2}=T$ (as in Subsection 1.4.1), but $U$ is not identically true. Let $\mathcal{F}=\mathcal{F}_{k_{2}+1}^{\sigma_{2}, U}$ and note that $\left(\sigma_{2}, \eta\right)$ is still a type extension of $\sigma_{1}$ but now $u=\sum_{F \in \mathcal{F}} F \neq 1_{\sigma_{2}}$.

In this special case, the interpretation homomorphism $\pi^{(U, I)}$ is denoted $\pi^{\mathcal{F}, \eta}$ (note that $U$ is uniquely determined by $\mathcal{F} \subset \mathcal{F}_{k_{2}+1}^{\sigma_{2}}$ up to equivalence of formulas), or even by $\pi^{F, \eta}$, when $\mathcal{F}$ consists of a single flag $F$ (dropping $\eta$ from notation when $\sigma_{1}=0$ ).

The intuition behind $\pi^{\mathcal{F}}$ is the following (again we suppose $\eta(i)=i$ for every $i \in\left[k_{1}\right]$ for simplicity).

Suppose that we have a homomorphism $\phi \in \operatorname{Hom}^{+}\left(\mathcal{A}^{\sigma_{2}}, \mathbb{R}\right)$ and suppose $F_{1}$ is a $\sigma_{1}$-flag of size $\ell_{1}$.

Thinking of $\phi$ as a large $\sigma_{2}$-flag, let $c_{1}, c_{2}, \ldots, c_{k_{2}}$ be the labelled vertices of $\phi$ (with each $c_{i}$ having label $i$ ) and let $W$ be the of the set of vertices $x$ of $\phi$ such that $U(x)$ holds (note that $U$ may involve the constants $c_{1}, c_{2}, \ldots, c_{k_{2}}$ ).

Suppose that we are interested in computing the density of $F_{1}$ inside $^{4} W$. To do this, we consider the following random experiment. We pick uniformly at random $d=\ell_{1}-k_{1}$ vertices $\boldsymbol{v}_{\mathbf{1}}, \boldsymbol{v}_{\mathbf{2}}, \ldots, \boldsymbol{v}_{\boldsymbol{d}}$ in the unlabelled part of $\phi$ and the sought density is

$$
\begin{aligned}
& \left.\mathbb{P}\left[\left\{\boldsymbol{v}_{\mathbf{1}}, \ldots, \boldsymbol{v}_{\boldsymbol{d}}\right\} \cup\left\{c_{1}, \ldots, c_{k_{1}}\right\} \text { is an occurrence of } F_{1} \text { in } \phi \mid\left\{\boldsymbol{v}_{\mathbf{1}}, \ldots, \boldsymbol{v}_{\boldsymbol{d}}\right\} \subset W\right\}\right] \\
& =\frac{\left.\mathbb{P}\left[\left\{\boldsymbol{v}_{\mathbf{1}}, \ldots, \boldsymbol{v}_{\boldsymbol{d}}\right\} \cup\left\{c_{1}, \ldots, c_{k_{1}}\right\} \text { is an occurrence of } F_{1} \text { in } \phi \wedge\left\{\boldsymbol{v}_{\mathbf{1}}, \ldots, \boldsymbol{v}_{\boldsymbol{d}}\right\} \subset W\right\}\right]}{\left.\mathbb{P}\left[\left\{\boldsymbol{v}_{\mathbf{1}}, \ldots, \boldsymbol{v}_{\boldsymbol{d}}\right\} \subset W\right\}\right]} \\
& =\frac{\sum_{F_{2} \in \mathcal{F}_{d+k_{2}}^{\sigma_{2}, U}: I^{\prime}\left(F_{2}\right) \cong F_{1}} \phi\left(F_{2}\right)}{\phi\left(u^{d}\right)} \\
& =\phi\left(\pi^{\mathcal{F}, \eta}\left(F_{1}\right)\right) \text {. }
\end{aligned}
$$

Therefore the sought density is given by the composition of $\phi$ with $\pi^{\mathcal{F}, \eta}$.

The next example is presented using this informal thought of homomorphisms as large flags.

Example 1.4.10. In the Theory of Graphs, if $\phi \in \operatorname{Hom}^{+}\left(\mathcal{A}^{1}, \mathbb{R}\right)$ and $f \in \mathcal{A}^{0}$, then

\footnotetext{
${ }^{4}$ We will also suppose this makes sense, which translates to the size of $W$ being non-negligible.
} 
- $\phi\left(\pi^{K_{2}^{1}}(f)\right)$ measures the density of $f$ in the neighbourhood of the labelled vertex of $\phi$;

- $\phi\left(\pi^{\bar{K}_{2}}(f)\right)$ measures the density of $f$ in the non-neighbourhood of the labelled vertex of $\phi$ (i.e., the set of vertices not adjacent to the labelled vertex);

On the other hand, if $\psi \in \operatorname{Hom}^{+}\left(\mathcal{A}^{E}, \mathbb{R}\right)$, then

- $\psi\left(\pi^{P_{3}^{E}}(f)\right)$ measures the density of $f$ in the common non-neighbourhood of the two labelled vertices of $\psi$ (i.e., the set of vertices that are not adjacent to any labelled vertex);

- $\psi\left(\pi^{P_{3}^{E, c}}(f)\right)$ measures the density of $f$ in the set of vertices of $\psi$ that are adjacent to the vertex with label 1 but not to the vertex with label 2;

- $\psi\left(\pi^{K_{3}^{E}}(f)\right)$ measures the density of $f$ in the common neighbourhood of the two labelled vertices of $\psi$;

- $\left.\psi\left(\pi^{\left\{{\overline{P_{3}}}^{E}\right.}, K_{3}^{E}\right\}(f)\right)$ measures the density of $f$ in the sameness set of the two labelled vertices (that is, the set of vertices that are either adjacent to both labelled vertices or non-adjacent to both labelled vertices).

The intuition for $\pi^{\mathcal{F}}$ for other $\mathcal{F}$ is analogous to the above.

For other examples, see Remarks 2.2.4 and 2.4.7.

As a final (but important) remark of this subsection, note that although Theorems 1.4.4 and 1.4.7 and Corollary 1.4.5 give a nice description of the behaviour of the upward operator $\pi^{\sigma, \eta}$, they also prove that this operator does not add any new information that was not already available via homomorphism extensions. However, this is no longer true for the interpretation homomorphisms $\pi^{\mathcal{F}, \eta}$ and this is the essence of the so called inductive method in flag algebras.

Let us now present the idea of the inductive method in the particular case of $T_{\text {Graph }}$ and $\pi^{K_{2}^{1}}$.

Suppose that we know that for a given $f \in \mathcal{A}^{0}$, we have $\phi(f) \geqslant 0$ for every homomorphism $\phi \in \operatorname{Hom}^{+}\left(\mathcal{A}^{0}, \mathbb{R}\right)$. Interpreting a homomorphism $\psi \in \operatorname{Hom}^{+}\left(\mathcal{A}^{0}, \mathbb{R}\right)$ as a large graph, we can consider a vertex $\boldsymbol{v}$ of $\psi$ picked uniformly at random $\left(\boldsymbol{\psi}^{\mathbf{1}}\right)$ and we know that the density of $f$ in the neighbourhood of $v$ must be non-negative (i.e., we have $\boldsymbol{\psi}^{\mathbf{1}}\left(\pi^{K_{2}^{1}}(f)\right) \geqslant 0$ a.s.). To retrieve some information about $\psi$ on $\mathcal{A}^{0}$, we can then average over the choice of $\boldsymbol{v}$, thus getting

$$
0 \leqslant \mathbb{E}\left[\boldsymbol{\psi}^{\mathbf{1}}\left(\pi^{K_{2}^{1}}(f)\right)\right]=\frac{\psi\left(\llbracket \pi^{K_{2}^{1}}(f) \rrbracket_{1}\right)}{\psi\left(\llbracket 1_{1} \rrbracket_{1}\right)},
$$

for every homomorphism $\psi \in \operatorname{Hom}^{+}\left(\mathcal{A}^{0}, \mathbb{R}\right)$.

Theorems 2.2.2, 2.4.8 and 2.4.9 will use this idea of the inductive method in the Theory of Tournaments.

\subsection{3 "Genuine" interpretations}

Finally, we are interested in "genuine" interpretations, that is, when $T_{1}$ and $T_{2}$ are different theories (and can even be over different languages). However, we will suppose that $U \equiv T$.

In this framework, the interpretation homomorphisms allow us to translate statements of one theory to another. 
The first two examples (1.4.11 and 1.4.12) are instances of global interpretations, in the sense that the predicate symbols of $T_{1}$ are translated to open formulas not involving constants of $T_{2}$. In this case, for every non-degenerate type $\sigma_{2}$ of $T_{2}$, we have a uniquely defined non-degenerate type $\sigma_{1}=I\left(\sigma_{2}\right)$ of $T_{1}$.

The final example (1.4.13) is an instance of a local interpretation since the translation takes predicate symbols to formulas involving constants of $T_{2}$, so the interpretation is heavily influenced by these few labelled vertices (hence the name "local").

Example 1.4.11. Let $T_{1}=T_{\text {Graph }}$ and $T_{2}=T_{\text {Digraph }}$ or $T_{2}=T_{\text {Tournaments. }}$.

- The orientation-erasing interpretation $\left(\top, I_{\mathrm{OE}}\right): T_{1} \rightsquigarrow T_{2}$, given by

$$
I_{\mathrm{OE}}(e)\left(x_{1}, x_{2}\right)=a\left(x_{1}, x_{2}\right) \vee a\left(x_{2}, x_{1}\right),
$$

gives rise to a algebra homomorphisms $\pi^{\left(\top, I_{\mathrm{OE}}\right)}: \mathcal{A}^{\sigma_{1}}\left[T_{1}\right] \rightarrow \mathcal{A}^{\sigma_{2}}\left[T_{2}\right]$ whenever the graph obtained by substituting every arc of $\sigma_{2}$ by an edge and removing multiplicities yields a graph isomorphic to $\sigma_{1}$;

- The strict orientation-erasing interpretation $\left(T, I_{\mathrm{SOE}}\right): T_{1} \rightsquigarrow T_{2}$, given by

$$
I_{\mathrm{SOE}}(e)\left(x_{1}, x_{2}\right)=a\left(x_{1}, x_{2}\right) \underline{\vee} a\left(x_{2}, x_{1}\right),
$$

gives rise to a algebra homomorphisms $\pi^{\left(\top, I_{\mathrm{SOE}}\right)}: \mathcal{A}^{\sigma_{1}}\left[T_{1}\right] \rightarrow \mathcal{A}^{\sigma_{2}}\left[T_{2}\right]$ whenever the graph obtained by removing anti-parallel arcs and substituting every remaining arc of $\sigma_{2}$ by an edge yields a graph isomorphic to $\sigma_{1}$;

- The anti-parallel arc interpretation $\left(\top, I_{\mathrm{AP}}\right): T_{1} \rightsquigarrow T_{2}$, given by

$$
I_{\mathrm{AP}}(e)\left(x_{1}, x_{2}\right)=a\left(x_{1}, x_{2}\right) \wedge a\left(x_{2}, x_{1}\right),
$$

gives rise to a algebra homomorphisms $\pi^{\left(\top, I_{\mathrm{AP}}\right)}: \mathcal{A}^{\sigma_{1}}\left[T_{1}\right] \rightarrow \mathcal{A}^{\sigma_{2}}\left[T_{2}\right]$ whenever the graph over $V\left(\sigma_{2}\right)$ that has edges precisely between pairs having anti-parallel arcs in $\sigma_{2}$ is isomorphic to $\sigma_{1}$.

Example 1.4.12. Let $T_{1}=T_{\text {Graph }}$ or $T_{1}=T_{\text {Graphofinversions }}$ and $T_{2}=T_{\text {Perm }}$. The inversion interpretation $\left(\top, I_{\mathrm{Inv}}\right):$, $\rightsquigarrow$ given by

$$
I_{\text {Inv }}(e)\left(x_{1}, x_{2}\right)=\left(a\left(x_{1}, x_{2}\right) \Longleftrightarrow b\left(x_{2}, x_{1}\right)\right),
$$

gives rise to an algebra homomorphisms $\pi^{\left(\top, I_{\text {Inv }}\right)}: \mathcal{A}^{\sigma_{1}}\left[T_{1}\right] \rightarrow \mathcal{A}^{\sigma_{2}}\left[T_{2}\right]$ whenever $\sigma_{1}$ is isomorphic to the graph of inversions $G_{\sigma_{2}}$ of $\sigma_{2}$.

Note that if $G$ is a graph that is not (isomorphic to) a graph of inversions (of some permutation), then $\pi^{\left(\top, I_{\text {Inv }}\right)}(G)=0$.

Example 1.4.13 ([DCF00]). Let $T_{1}=T_{\text {Graph }}, T_{2}=T_{3 \text {-hypergraph }}$ and $\sigma_{1}=0$. Let also $\sigma_{2}=1$ be the unique type of size 1 in $T_{3}$-hypergraph and $c_{1}$ be the constant symbol of $T_{2}^{\sigma_{2}}$.

The link interpretation $\left(\top, I_{\text {link }}\right): T_{1} \rightsquigarrow T_{2}$, given by

$$
I_{\text {link }}(e)\left(x_{1}, x_{2}\right)=e\left(c_{1}, x_{1}, x_{2}\right) \text {, }
$$

gives rise to the algebra homomorphism $\pi^{\left(\top, I_{\text {link }}\right)}: \mathcal{A}^{0}\left[T_{1}\right] \rightarrow \mathcal{A}^{1}\left[T_{2}\right]$.

As a final remark of this section, let us mention that an analogous theorem to Theorem 1.4.7 also holds for "genuine" interpretations and we refer the interested reader to [Raz07, Theorem 4.1]. 


\section{Chapter 2}

\section{Minimization of transitive tournaments and quasi-randomness}

The first part of this chapter is devoted to a novel result on minimization of density of transitive tournaments. More specifically, we will prove in Section 2.2 that the density of a transitive tournament of size at least 4 is asymptotically minimized only by quasi-random sequences. But before presenting this result, we will make a brief introduction to the theory of tournament quasi-randomness (Section 2.1).

The second part of this chapter is devoted to a generalization of this minimization result to several 0-flag algebra elements. More specifically, in Section 2.4, we will provide a way of obtaining several fixed $f \in \mathcal{A}^{0}$ such that $\phi(f)$ is minimized $\left(\phi \in \operatorname{Hom}^{+}\left(\mathcal{A}^{0}, \mathbb{R}\right)\right)$ only by the quasi-random homomorphism $\phi_{\mathrm{qr}}$.

As a byproduct of these results and their proofs, several novel characterizations of tournament quasi-randomness will arise (these will be scattered across Sections 2.2, 2.3 and 2.4).

Throughout this chapter, all flag algebra terms and symbols will be relative to the theory $T_{\text {Tournaments. }}$.

\subsection{An introduction to tournament quasi-randomness}

One of the most basic models of a random tournament is the following.

Definition 2.1.1. For every $n \in \mathbb{N}$, the random tournament of size $n$, denoted by $\boldsymbol{R}_{\boldsymbol{n}, \mathbf{1} / \mathbf{2}}$, is the (random) tournament with vertex set

$$
V\left(\boldsymbol{R}_{n, \mathbf{1} / \mathbf{2}}\right)=[n]
$$

and such that, for every pair of distinct vertices $x$ and $y$, we have

$$
\mathbb{P}\left[x y \in A\left(\boldsymbol{R}_{\boldsymbol{n}, \mathbf{1 / 2}}\right)\right]=\frac{1}{2},
$$

independently of all other pairs of vertices.

Since the arc orientations of $\boldsymbol{R}_{\boldsymbol{n}, \mathbf{1} / \mathbf{2}}$ are independent, it is easy to see that the expected value of the density of any fixed tournament $T$ of size $\ell \leqslant n$ in $\boldsymbol{R}_{\boldsymbol{n , 1 / 2}}$ is

$$
\mathbb{E}\left[p\left(T ; \boldsymbol{R}_{\boldsymbol{n}, \mathbf{1 / 2}}\right)\right]=\frac{\ell !}{|\operatorname{Aut}(T)| 2^{\left(\begin{array}{l}
\ell \\
2
\end{array}\right)}} .
$$


It is an exercise on probability concentration to see the following property of the sequence of random tournaments.

Proposition 2.1.2. The sequence of random tournaments of increasing sizes is convergent with probability 1 , that is, for every fixed tournament $T \in \mathcal{F}_{\ell}^{0}$ of size $\ell$, we have

$$
\lim _{n \rightarrow \infty} p\left(T ; \boldsymbol{R}_{\boldsymbol{n , \mathbf { 1 } / \mathbf { 2 }}}\right)=\mathbb{E}\left[p\left(T ; \boldsymbol{R}_{\boldsymbol{\ell , \mathbf { 1 } / \mathbf { 2 }}}\right)\right]=\frac{\ell !}{|\operatorname{Aut}(T)| 2^{\left(\begin{array}{c}
\ell \\
2
\end{array}\right)}} \text { a.s. }
$$

This proposition allows us to define the quasi-random homomorphism $\phi_{\mathrm{qr}}$ as the a.s. limit of the sequence of random tournaments, that is $\phi_{\mathrm{qr}}$ is the element of $\operatorname{Hom}^{+}\left(\mathcal{A}^{0}, \mathbb{R}\right)$ such that

$$
\phi_{\mathrm{qr}}(T)=\frac{\ell !}{|\operatorname{Aut}(T)| 2^{\left(\begin{array}{l}
\ell \\
2
\end{array}\right)}}
$$

for every $T \in \mathcal{F}_{\ell}^{0}$.

The next theorem by Chung and Graham inaugurated the theory of tournament quasirandomness. But to properly state it in the language of flag algebras, we must first define some flags (see Figure 2.1 and Tables 2.1 and 2.2).

Definition 2.1.3. For every $k \in \mathbb{N}$, the transitive tournament of size $k$, denoted by $\operatorname{Tr}_{k}$, is the only tournament of size $k$ that satisfies the axiom of transitivity, that is, it is the only tournament of size $k$ such that

$$
\forall x \forall y \forall z,\left(x y \in A\left(\operatorname{Tr}_{k}\right) \wedge y z \in A\left(\operatorname{Tr}_{k}\right) \Longrightarrow x z \in A\left(\operatorname{Tr}_{k}\right)\right)
$$

The tournament $\vec{C}_{3}$ is the 3-cycle and $R_{4}$ is the only tournament of size 4 that has a 4-cycle.

The type $A$ is the type of size 2 where the vertex with label 1 beats the vertex with label 2.

Finally, we define the following A-flags of size 3.

- The flag $O^{A}$, in which the unlabelled vertex is beaten by both labelled vertices $(O$ stands for common outneighbourhood);

- The flag $I^{A}$, in which the unlabelled vertex beats both labelled vertices (I stands for common inneighbourhood);

- The flag $\operatorname{Tr}_{3}^{A}$, which is the only remaining A-flag whose underlying tournament is $\operatorname{Tr}_{3}$ (that is, we have $\left.\left.\left(\operatorname{Tr}_{3}^{A}\right)\right|_{0}=\operatorname{Tr}_{3}\right)$;

- The flag $\vec{C}_{3}^{A}$, which is the only $A$-flag whose underlying tournament is $\vec{C}_{3}$ (that is, we have $\left.\left.\left(\vec{C}_{3}^{A}\right)\right|_{0}=\vec{C}_{3}\right)$.

Theorem 2.1.4 (Chung-Graham [CG91, Theorem 1]). The following properties are equivalent for a homomorphism $\phi \in \operatorname{Hom}^{+}\left(\mathcal{A}^{0}, \mathbb{R}\right)$.

$P_{1}: \phi=\phi_{\mathrm{qr}} ;$

$P_{2}$ : $\phi$ minimizes the density of $\operatorname{Tr}_{4}+R_{4}$ with the value $3 / 4$, that is, we have

$$
\phi\left(\operatorname{Tr}_{4}+R_{4}\right)=\min \left\{\psi\left(\operatorname{Tr}_{4}+R_{4}\right): \psi \in \operatorname{Hom}^{+}\left(\mathcal{A}^{0}, \mathbb{R}\right)\right\}=\frac{3}{4} ;
$$



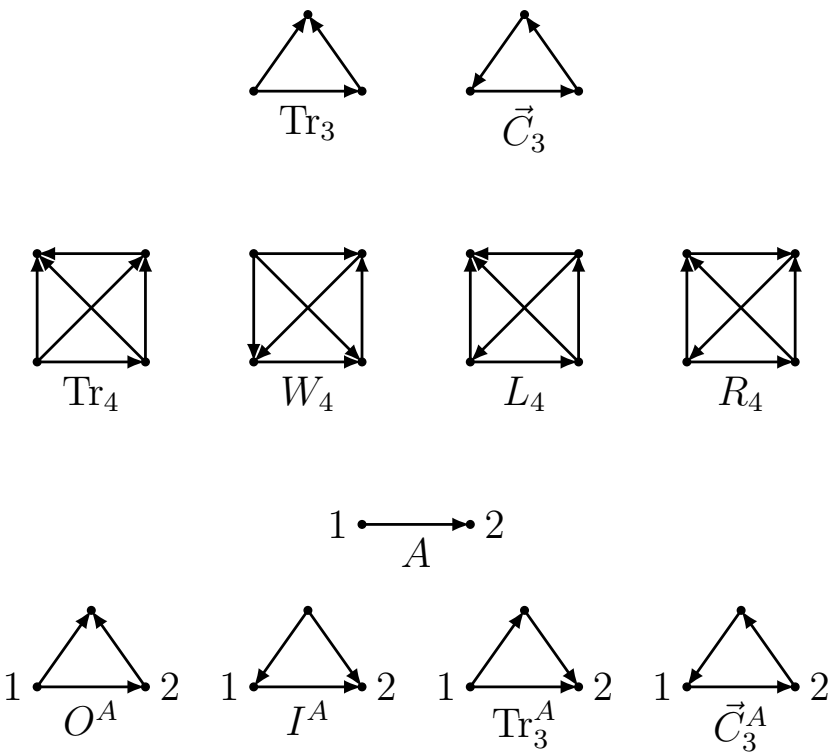

Figure 2.1: Tournaments of sizes 3 and 4, type $A$ and $A$-flags of size 3.

\begin{tabular}{c|cccccc} 
& $\operatorname{Tr}_{3}$ & $\vec{C}_{3}$ & $\operatorname{Tr}_{4}$ & $R_{4}$ & $W_{4}$ & $L_{4}$ \\
\hline $\operatorname{Tr}_{1}$ & 1 & 1 & 1 & 1 & 1 & 1 \\
$\operatorname{Tr}_{2}$ & 1 & 1 & 1 & 1 & 1 & 1 \\
$\operatorname{Tr}_{3}$ & 1 & 0 & 1 & $\frac{1}{2}$ & $\frac{3}{4}$ & $\frac{3}{4}$ \\
$\vec{C}_{3}$ & 0 & 1 & 0 & $\frac{1}{2}$ & $\frac{1}{4}$ & $\frac{1}{4}$
\end{tabular}

Table 2.1: Joint densities $p(A ; B)$ in the Theory of tournaments in type 0 , where $A$ is given by the leftmost entry of the line and $B$ by the topmost entry of the column.

$$
\begin{aligned}
& P_{3}: \boldsymbol{\phi}^{\boldsymbol{A}}\left(O^{A}+I^{A}\right)=1 / 2 \text { a.s.; } \\
& P_{4}: \boldsymbol{\phi}^{\boldsymbol{A}}\left(O^{A}\right)=1 / 4 \text { a.s. }
\end{aligned}
$$

Remark 2.1.5. The original theorem by Chung and Graham is not in the language of flag algebras (not even uses any notion of limit of tournament sequences) and actually proves the equivalence of 11 quasi-random properties ( $P_{1}$ through $P_{11}$, whose notation we preserved for the first four).

Remark 2.1.6. By flipping all arcs in $P_{4}$ (and swapping the labels), it is easy to see that

$$
P_{4}^{\prime}: \phi^{A}\left(I^{A}\right)=1 / 4 \text { a.s. }
$$

is also a quasi-random property, i.e., we have that $P_{4}^{\prime}$ is equivalent to $P_{1}$.

Furthermore, since $O^{A}+I^{A}=1_{0}-\operatorname{Tr}_{3}^{A}-\vec{C}_{3}^{A}$, we trivially have that

$$
P_{3}^{\prime}: \phi^{A}\left(\operatorname{Tr}_{3}^{A}+\vec{C}_{3}^{A}\right)=1 / 2 \text { a.s. }
$$

is also a quasi-random property. 


\begin{tabular}{c|ccccc} 
& $1_{A}$ & $O^{A}$ & $I^{A}$ & $\operatorname{Tr}_{3}^{A}$ & $\vec{C}_{3}^{A}$ \\
\hline$\llbracket \cdot \rrbracket_{A}$ & $\frac{1}{2} \operatorname{Tr}_{2}$ & $\frac{1}{6} \operatorname{Tr}_{3}$ & $\frac{1}{6} \operatorname{Tr}_{3}$ & $\frac{1}{6} \operatorname{Tr}_{3}$ & $\frac{1}{2} \vec{C}_{3}$
\end{tabular}

Table 2.2: Downward operator in the Theory of tournaments from type $A$ to type 0 (the function $\eta$ is omitted from the notation).

\subsection{Minimization of transitive tournaments}

Before presenting the main result of this section, let us define some more flags.

Definition 2.2.1. Let 1 denote the only type of size 1 , let $\alpha$ denote the 1 denote the 1-flag of size 2 in which the labelled vertex beats the unlabelled vertex and let $\beta$ denote the only other 1-flag of size 2 (see Figure 2.2).

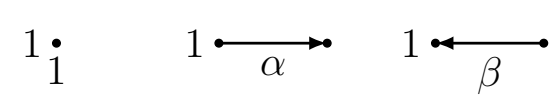

Figure 2.2: Type 1 and flags of size 2 over the type 1.

We now have all the tools necessary to prove the following result.

Theorem 2.2.2 (C.-Razborov [CR15, Theorem 2.1]). If $\phi \in \operatorname{Hom}^{+}\left(\mathcal{A}^{0}, \mathbb{R}\right)$, then

$$
\phi\left(\operatorname{Tr}_{k}\right) \geqslant \frac{k !}{2^{\left(\begin{array}{c}
k \\
2
\end{array}\right)}}
$$

Furthermore, if $k \geqslant 4$, then equality holds if and only if $\phi=\phi_{\mathrm{qr}}$, i.e., minimization of the density of $\operatorname{Tr}_{k}$ is a quasi-random property (for every $k \geqslant 4$ ).

Remark 2.2.3. The cases $k=0,1,2$ of this result are trivial, the case $k=3$ was already known by Chung and Graham [CG91, Fact 1], and the case $k=4$ was already known by Griffiths [Gri13, P1 of Theorem 1.1 and Proposition 3.1i].

Proof. The cases $k=0,1,2$ follow from $1_{0}=\operatorname{Tr}_{0}=\operatorname{Tr}_{1}=\operatorname{Tr}_{2}$ and the fact that the right-hand side of inequality (2.1) is 1 for $k=0,1,2$.

For the case $k=3$, let $\operatorname{Tr}_{3}^{\mathrm{W}}$ denote the 1-flag obtained from $\operatorname{Tr}_{3}$ by labelling the vertex with maximum outdegree (that is, the winner). Note that $\alpha^{2}=\operatorname{Tr}_{3}^{\mathrm{W}}$ and that $\llbracket \operatorname{Tr}_{3}^{\mathrm{W}} \rrbracket_{1}=\operatorname{Tr}_{3} / 3$.

Since $\llbracket 1_{1} \rrbracket_{1}=1_{0}$, we have

$$
\phi\left(\operatorname{Tr}_{3}\right)=3 \phi\left(\llbracket \alpha^{2} \rrbracket_{1}\right)=3 \mathbb{E}\left[\phi^{\mathbf{1}}(\alpha)^{2}\right] \geqslant 3 \mathbb{E}\left[\phi^{\mathbf{1}}(\alpha)\right]^{2}=3 \phi\left(\llbracket \alpha \rrbracket_{1}\right)^{2}=\frac{3}{4}=\frac{3 !}{2^{\left(\begin{array}{c}
3 \\
2
\end{array}\right)}} .
$$

We now proceed by induction. Suppose $k \geqslant 4$ and that the result is valid for $k-2$ and let $\operatorname{Tr}_{k}^{\mathrm{W} 2}$ denote the $A$-flag obtained from $\operatorname{Tr}_{k}$ by labelling the vertex with maximum outdegree with 1 and the vertex with second largest outdegree with 2 (that is, the winner and the runner-up respectively). Note that $\llbracket \operatorname{Tr}_{k}^{\mathrm{W} 2} \rrbracket_{A}=\operatorname{Tr}_{k} /(k(k-1))$ and that

$$
\pi^{O^{A}}\left(\operatorname{Tr}_{k-2}\right)=\frac{\operatorname{Tr}_{k}^{\mathrm{W} 2}}{\left(O^{A}\right)^{k-2}}
$$


Since $\llbracket 1_{A} \rrbracket_{A}=1_{0} / 2$, we have

$$
\begin{aligned}
\phi\left(\operatorname{Tr}_{k}\right) & =k(k-1) \phi\left(\llbracket \pi^{O^{A}}\left(\operatorname{Tr}_{k-2}\right)\left(O^{A}\right)^{k-2} \rrbracket_{A}\right) \\
& =\frac{k(k-1)}{2} \mathbb{E}\left[\boldsymbol{\phi}^{\boldsymbol{A}}\left(\pi^{O^{A}}\left(\operatorname{Tr}_{k-2}\right)\right) \boldsymbol{\phi}^{\boldsymbol{A}}\left(O^{A}\right)^{k-2}\right] .
\end{aligned}
$$

Since $\boldsymbol{\phi}^{\boldsymbol{A}}\left(O^{A}\right)>0$ implies that $\boldsymbol{\phi}^{\boldsymbol{A}} \circ \pi^{O^{A}} \in \operatorname{Hom}^{+}\left(\mathcal{A}^{0}, \mathbb{R}\right)$, by the inductive hypothesis, we have

$$
\mathbb{E}\left[\phi^{\boldsymbol{A}}\left(\pi^{O^{A}}\left(\operatorname{Tr}_{k-2}\right)\right) \boldsymbol{\phi}^{\boldsymbol{A}}\left(O^{A}\right)^{k-2}\right] \geqslant \frac{(k-2) !}{2^{\left(\begin{array}{c}
k-2 \\
2
\end{array}\right)}} \mathbb{E}\left[\boldsymbol{\phi}^{\boldsymbol{A}}\left(O^{A}\right)^{k-2}\right]
$$

Now, by Jensen's inequality, we have

$$
\mathbb{E}\left[\boldsymbol{\phi}^{\boldsymbol{A}}\left(O^{A}\right)^{k-2}\right] \geqslant \mathbb{E}\left[\boldsymbol{\phi}^{\boldsymbol{A}}\left(O^{A}\right)\right]^{k-2}=\left(2 \phi\left(\llbracket O^{A} \rrbracket_{A}\right)\right)^{k-2}=\left(\frac{\phi\left(\operatorname{Tr}_{3}\right)}{3}\right)^{k-2} .
$$

Since we already proved that $\phi\left(\operatorname{Tr}_{3}\right) \geqslant 3 / 4$, we get

$$
\phi\left(\operatorname{Tr}_{k}\right) \geqslant \frac{k(k-1)}{2} \cdot \frac{(k-2) !}{2^{\left(\begin{array}{c}
k-2 \\
2
\end{array}\right)}} \cdot\left(\frac{\phi\left(\operatorname{Tr}_{3}\right)}{3}\right)^{k-2} \geqslant \frac{k !}{2^{\left(\begin{array}{c}
k \\
2
\end{array}\right)}} .
$$

For the second part of the theorem, note that if equality holds for $\phi$ in (2.1), then we must have equality throughout the proof of the case $k \geqslant 4$.

In particular, since we used Jensen's inequality on (2.2) with the function $x \mapsto x^{k-2}$, which is strictly convex (for $x \geqslant 0$ ), we have that $\phi^{\boldsymbol{A}}\left(O^{A}\right)$ is almost surely a constant $C$.

Furthermore, equality in (2.3) implies that $\phi\left(\operatorname{Tr}_{3}\right)=3 / 4$, hence

$$
C=\mathbb{E}\left[\phi^{\boldsymbol{A}}\left(O^{A}\right)\right]=2 \phi\left(\llbracket O^{A} \rrbracket_{A}\right)=\frac{\phi\left(\operatorname{Tr}_{3}\right)}{3}=\frac{1}{4}
$$

Therefore $\phi$ satisfies $P_{4}$, hence $\phi=\phi_{\mathrm{qr}}$.

Remark 2.2.4. Recall the intuition presented in Subsection 1.4 .2 and note that $\phi\left(\pi^{O^{A}}(f)\right)$ measures the density of $f \in \mathcal{A}^{0}$ in the common outneighbourhood of the labelled vertices.

Define the transitive homomorphism as the limit $\phi_{\operatorname{Tr}} \in \operatorname{Hom}^{+}\left(\mathcal{A}^{0}, \mathbb{R}\right)$ of the sequence of transitive tournaments $\left(\operatorname{Tr}_{k}\right)_{k \in \mathbb{N}}$ (it is easy to see that this sequence is convergent).

The following immediate corollary completely closes the problem of minimizing the density of a single fixed tournament asymptotically.

Corollary 2.2.5. If $T \in \mathcal{F}_{\ell}^{0}$ is a tournament of size $\ell$, then

$$
\min \left\{\phi(T): \phi \in \operatorname{Hom}^{+}\left(\mathcal{A}^{0}, \mathbb{R}\right)\right\}= \begin{cases}\frac{\ell !}{2^{\left(\begin{array}{l}
\ell \\
2
\end{array}\right)},} & \text { if } T \text { is transitive }\left(T \cong \operatorname{Tr}_{\ell}\right) \\
0, & \text { if } T \text { is not transitive }\end{cases}
$$

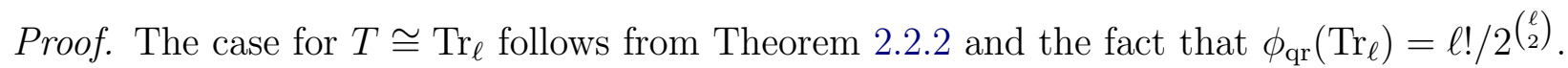

On the other hand, if $T$ is not transitive, then $\phi_{\operatorname{Tr}}(T)=0$. 


\subsection{Other quasi-random properties} size 3 .

In this section, we present some new quasi-random properties concerning the $A$-flags of

Theorem 2.3.1 (C.-Razborov [CR15, Theorems 3.1 and 3.2]). Given a homomorphism $\phi \in$ $\operatorname{Hom}^{+}\left(\mathcal{A}^{0}, \mathbb{R}\right)$, the following properties are equivalent.

- $P_{1}: \phi=\phi_{\mathrm{qr}}$;

- $\phi^{A}\left(\operatorname{Tr}_{3}^{A}\right)=1 / 4$ a.s.;

- $\phi^{A}\left(\vec{C}_{3}^{A}\right)=1 / 4$ a.s. .

Proof. Let us first prove the equivalence of the first two items (which will follow from an argument very similar to the second part of the proof of Theorem 2.2.2).

Let $\operatorname{Tr}_{4}^{A}$ denote the $A$-flag obtained from $\operatorname{Tr}_{4}$ by labelling the vertex with maximum outdegree with 1 and the vertex with outdegree 0 with 2 (that is, the winner and the loser respectively). Note that $\left(\operatorname{Tr}_{3}^{A}\right)^{2}=\operatorname{Tr}_{4}^{A}$ and $\llbracket \operatorname{Tr}_{4}^{A} \rrbracket_{A}=\operatorname{Tr}_{4} / 12$.

Since $\llbracket 1_{A} \rrbracket_{A}=1_{0} / 2$, we have

$$
\begin{aligned}
\phi\left(\operatorname{Tr}_{4}\right) & =12 \phi\left(\llbracket\left(\operatorname{Tr}_{3}^{A}\right)^{2} \rrbracket_{A}\right)=6 \mathbb{E}\left[\phi^{\boldsymbol{A}}\left(\operatorname{Tr}_{3}^{A}\right)^{2}\right] \\
& \geqslant 6 \mathbb{E}\left[\phi^{\boldsymbol{A}}\left(\operatorname{Tr}_{3}^{A}\right)\right]^{2}=6\left(2 \phi\left(\llbracket \operatorname{Tr}_{3}^{A} \rrbracket_{A}\right)\right)^{2}=\frac{2}{3} \phi\left(\operatorname{Tr}_{3}\right)^{2} .
\end{aligned}
$$

By Theorem 2.2.2, we have $\phi\left(\operatorname{Tr}_{3}\right) \geqslant 3 / 4$, hence

$$
\phi\left(\operatorname{Tr}_{4}\right) \geqslant \frac{2}{3} \phi\left(\operatorname{Tr}_{3}\right)^{2} \geqslant \frac{3}{8}=\frac{4 !}{2^{\left(\begin{array}{c}
4 \\
2
\end{array}\right)}}
$$

Since we used Jensen's inequality with the function $x \mapsto x^{2}$, which is strictly convex, we have that equality holds if and only if we have almost surely

$$
\phi^{\boldsymbol{A}}\left(\operatorname{Tr}_{3}^{A}\right)=\mathbb{E}\left[\phi^{\boldsymbol{A}}\left(\operatorname{Tr}_{3}^{A}\right)\right]=\frac{\phi\left(\operatorname{Tr}_{3}\right)}{3}=\frac{1}{4}
$$

Hence, by the second part of Theorem 2.2.2, we have

$$
\phi^{A}\left(\operatorname{Tr}_{3}^{A}\right)=\frac{1}{4} \text { a.s. } \Longleftrightarrow \phi\left(\operatorname{Tr}_{4}\right)=\frac{3}{8} \Longleftrightarrow \phi=\phi_{\mathrm{qr}} .
$$

Now we will prove the other implications.

First note that, from the first part of the proof and Properties $P_{4}$ and $P_{4}^{\prime}$, we have

$$
\phi_{\mathbf{q r}}^{\boldsymbol{A}}\left(O^{A}\right)=\phi_{\mathbf{q r}}^{\boldsymbol{A}}\left(I^{A}\right)=\phi_{\mathbf{q r}}^{\boldsymbol{A}}\left(\operatorname{Tr}_{3}^{A}\right)=\frac{1}{4} \text { a.s. }
$$

Hence

$$
\phi_{\mathrm{qr}}^{A}\left(\vec{C}_{3}^{A}\right)=\phi_{\mathrm{qr}}^{A}\left(1_{A}-O^{A}-I^{A}-\vec{C}_{3}^{A}\right)=\frac{1}{4} \text { a.s. }
$$

It remains only to prove that $\phi^{A}\left(\vec{C}_{3}^{A}\right)=1 / 4$ a.s. implies $\phi=\phi_{\mathrm{qr}}$. 
Note that

$$
\phi\left(\vec{C}_{3}\right)=\frac{\phi\left(\llbracket \vec{C}_{3}^{A} \rrbracket_{A}\right)}{\phi\left(\llbracket 1_{A} \rrbracket_{A}\right)}=\mathbb{E}\left[\phi^{\boldsymbol{A}}\left(\vec{C}_{3}^{A}\right)\right]=\frac{1}{4} .
$$

Now, it is straightforward to check the following flag algebra equalities.

$$
R_{4}=12 \llbracket\left(\vec{C}_{3}^{A}\right)^{2} \rrbracket_{A} ; \quad \vec{C}_{3}=\frac{R_{4}}{2}+\frac{W_{4}}{4}+\frac{L_{4}}{4} .
$$

Using the first equality along with $\phi^{\boldsymbol{A}}\left(\vec{C}_{3}^{A}\right)=1 / 4$ a.s., we get

$$
\phi\left(R_{4}\right)=12 \phi\left(\llbracket\left(\vec{C}_{3}^{A}\right)^{2} \rrbracket_{A}\right)=6 \mathbb{E}\left[\phi^{A}\left(\vec{C}_{3}^{A}\right)^{2}\right]=\frac{3}{8} .
$$

And using the second equality, we get

$$
\phi\left(W_{4}+L_{4}\right)=4 \phi\left(\vec{C}_{3}\right)-2 \phi\left(R_{4}\right)=\frac{1}{4},
$$

hence

$$
\phi\left(\operatorname{Tr}_{4}+R_{4}\right)=1-\phi\left(W_{4}+L_{4}\right)=\frac{3}{4},
$$

that is, the homomorphism $\phi$ satisfies $P_{2}$.

Therefore $\phi=\phi_{\mathrm{qr}}$.

Before stating more quasi-random properties, let us first present another class of equivalent properties discovered by Chung and Graham that is strictly weaker than the quasi-random properties.

Proposition 2.3.2 (Chung-Graham [CG91, Theorem 2]). The following properties are equivalent for a homomorphism $\phi \in \operatorname{Hom}^{+}\left(\mathcal{A}^{0}, \mathbb{R}\right)$.

$Q_{1}: \phi\left(\operatorname{Tr}_{3}\right)=3 / 4$ and $\phi\left(\vec{C}_{3}\right)=1 / 4 ;$

$Q_{2}$ : $\phi$ maximizes the value of $\phi\left(\vec{C}_{3}\right)$;

$Q_{3}: \phi^{\mathbf{1}}(\alpha)=1 / 2$ a.s.

Remark 2.3.3. Note that a simple proof of this proposition in the language of flag algebras follows from an analysis of the case $k=3$ of the proof of Theorem 2.2.2: equality holds throughout the proof if and only if $\phi^{\mathbf{1}}(\alpha)=1 / 2$ a.s.

For another proof using the flag algebra semidefinite method, see Proposition B.5.

We call a homomorphism balanced if it satisfies any (and therefore all) of these properties.

Remark 2.3.4. This nomenclature stems from Property $Q_{3}$, which says that every sequence of tournaments $\left(T_{n}\right)_{n \in \mathbb{N}}$ converging to $\phi$ is almost balanced in the sense that all but $o\left(\left|T_{n}\right|\right)$ vertices of $T_{n}$ have outdegree $(1 / 2+o(1))\left|T_{n}\right|$.

Let us now add another property to the list of balanced properties.

Lemma 2.3.5 (C. [Cor15, Lemma 2.2]). For every homomorphism $\phi \in \operatorname{Hom}^{+}\left(\mathcal{A}^{0}, \mathbb{R}\right)$, we have

$$
\phi\left(\operatorname{Tr}_{4}\right) \geqslant \phi\left(R_{4}\right)
$$


with equality if and only if $\phi$ is balanced.

Proof. It is straightforward to check the following flag algebra equalities.

$$
\vec{C}_{3}=\frac{R_{4}}{2}+\frac{W_{4}}{4}+\frac{L_{4}}{4}=\frac{1_{0}-\left(\operatorname{Tr}_{4}-R_{4}\right)}{4} .
$$

The result now clearly follows from $Q_{1}$ and $Q_{2}$.

The following corollary of Theorem 2.3.1 and of Properties $P_{4}$ and $P_{4}^{\prime}$ says that even knowing only that one of $\phi^{\boldsymbol{A}}\left(O^{A}\right), \phi^{\boldsymbol{A}}\left(I^{A}\right)$ or $\phi^{\boldsymbol{A}}\left(\operatorname{Tr}_{3}^{A}\right)$ is deterministic is enough to force quasi-randomness.

Corollary 2.3.6. Given a homomorphism $\phi \in \operatorname{Hom}^{+}\left(\mathcal{A}^{0}, \mathbb{R}\right)$, the following properties are equivalent.

- $P_{1}: \phi=\phi_{\mathrm{qr}}$;

- $\operatorname{Var}\left[\boldsymbol{\phi}^{\boldsymbol{A}}\left(O^{A}\right)\right]=0$;

- $\operatorname{Var}\left[\phi^{\boldsymbol{A}}\left(I^{A}\right)\right]=0$;

- $\operatorname{Var}\left[\phi^{\boldsymbol{A}}\left(\operatorname{Tr}_{3}^{A}\right)\right]=0$.

Proof. First note that if $F \in\left\{O^{A}, I^{A}, \operatorname{Tr}_{3}^{A}\right\}$, then we have

$$
\mathbb{E}\left[\boldsymbol{\phi}^{\boldsymbol{A}}(F)\right]=\frac{\operatorname{Tr}_{3}}{3} ; \quad \mathbb{E}\left[\boldsymbol{\phi}^{\boldsymbol{A}}(F)^{2}\right]=\frac{\operatorname{Tr}_{4}}{6} .
$$

Hence

$$
\operatorname{Var}\left[\boldsymbol{\phi}^{\boldsymbol{A}}\left(O^{A}\right)\right]=\operatorname{Var}\left[\boldsymbol{\phi}^{\boldsymbol{A}}\left(I^{A}\right)\right]=\operatorname{Var}\left[\boldsymbol{\phi}^{\boldsymbol{A}}\left(\operatorname{Tr}_{3}^{A}\right)\right]
$$

which implies that the three last items are equivalent.

Since $P_{4}$ implies the second item, to complete the proof it is enough to prove that the three last items together imply $\phi=\phi_{\mathrm{qr}}$.

Suppose then that $\operatorname{Var}\left[\boldsymbol{\phi}^{\boldsymbol{A}}(F)\right]=0$ for every $F \in\left\{O^{A}, I^{A}, \operatorname{Tr}_{3}^{A}\right\}$ and note that we have $\phi^{\boldsymbol{A}}\left(O^{A}\right)=\phi^{\boldsymbol{A}}\left(I^{A}\right)$ a.s., hence

$$
0=\mathbb{E}\left[\left(\phi^{\boldsymbol{A}}\left(O^{A}\right)-\boldsymbol{\phi}^{\boldsymbol{A}}\left(I^{A}\right)\right)^{2}\right]=\frac{\operatorname{Tr}_{4}}{6}-\frac{R_{4}}{6},
$$

which implies that $\phi$ is balanced by Lemma 2.3.5.

By Proposition 2.3.2, we get that $\phi\left(\operatorname{Tr}_{3}\right)=3 / 4$, hence $\phi^{\boldsymbol{A}}\left(O^{A}\right)=1 / 4$ a.s., that is, the homomorphism $\phi$ satisfies $P_{4}$.

Therefore $\phi=\phi_{\mathrm{qr}}$.

One natural question that arises from the corollary above is what happens with the last $A$-flag $\vec{C}_{3}^{A}$ of size 3 ? The following corollary of Theorem 2.3.1 says that there are exactly two homomorphisms $\phi$ such that $\operatorname{Var}\left[\phi^{\boldsymbol{A}}\left(\vec{C}_{3}^{A}\right)\right]=0$.

Corollary 2.3.7. For every homomorphism $\phi \in \operatorname{Hom}^{+}\left(\mathcal{A}^{0}, \mathbb{R}\right)$, we have $\operatorname{Var}\left[\boldsymbol{\phi}^{\boldsymbol{A}}\left(\vec{C}_{3}^{A}\right)\right]=0$ if and only if $\phi \in\left\{\phi_{\mathrm{qr}}, \phi_{\mathrm{Tr}}\right\}$. 
Proof. We already know that $\operatorname{Var}\left[\boldsymbol{\phi}_{\mathbf{q r}}^{\boldsymbol{A}}\left(\vec{C}_{3}^{A}\right)\right]=0$. Note also that since $\mathbb{E}\left[\boldsymbol{\phi}_{\mathbf{T r}}^{\boldsymbol{A}}\left(\vec{C}_{3}^{A}\right)\right]=$ $\phi_{\operatorname{Tr}}\left(\vec{C}_{3}\right)=0$ we get $\operatorname{Var}\left[\phi_{\operatorname{Tr}}^{\boldsymbol{A}}\left(\vec{C}_{3}^{A}\right)\right]=0$.

It remains to prove that these are the only two homomorphisms with this property, so let $\phi \in \operatorname{Hom}^{+}\left(\mathcal{A}^{0}, \mathbb{R}\right)$ be such that $\operatorname{Var}\left[\phi^{A}\left(\vec{C}_{3}^{A}\right)\right]=0$.

Note that

$$
\operatorname{Var}\left[\phi^{\boldsymbol{A}}\left(\vec{C}_{3}^{A}\right)\right]=\frac{\phi\left(R_{4}\right)}{6}-\left(\phi\left(\vec{C}_{3}\right)\right)^{2}
$$

hence $\phi\left(R_{4}\right) / 6=\left(\phi\left(\vec{C}_{3}\right)\right)^{2}$.

On the other hand, since $\phi^{\boldsymbol{A}}\left(\vec{C}_{3}^{A}\right)$ is a.s. a constant, we have

$$
\frac{\phi\left(\operatorname{Tr}_{3}\right) \phi\left(\vec{C}_{3}\right)}{3}=\mathbb{E}\left[\phi^{A}\left(\operatorname{Tr}_{3}^{A}\right)\right] \mathbb{E}\left[\phi^{A}\left(\vec{C}_{3}^{A}\right)\right]=\mathbb{E}\left[\phi^{A}\left(\vec{C}_{3}^{A} \operatorname{Tr}_{3}^{A}\right)\right]=\frac{\phi\left(R_{4}\right)}{6}=\left(\phi\left(\vec{C}_{3}\right)\right)^{2}
$$

which implies that

$$
\phi\left(\vec{C}_{3}\right)\left(\phi\left(\vec{C}_{3}\right)-\frac{\phi\left(\operatorname{Tr}_{3}\right)}{3}\right)=0
$$

Therefore, either $\phi\left(\vec{C}_{3}\right)=0$ or $\phi\left(\vec{C}_{3}\right)=\phi\left(\operatorname{Tr}_{3}\right) / 3$. In the first case we get $\phi=\phi_{\operatorname{Tr}}$ and in the latter case, we get $\phi\left(\vec{C}_{3}\right)=1 / 4$, which implies

$$
\phi^{\boldsymbol{A}}\left(\vec{C}_{3}^{A}\right)=\mathbb{E}\left[\phi^{\boldsymbol{A}}\left(\vec{C}_{3}^{A}\right)\right]=\phi\left(\vec{C}_{3}\right)=\frac{1}{4} \text { a.s. }
$$

hence $\phi=\phi_{\mathrm{qr}}$ by Theorem 2.3.1.

\subsection{Further extremal quasi-randomness properties}

In this section, we will present a generalization of Theorem 2.2.2 to other 0-flag algebra elements, but to do so, we need to define some notation.

Definition 2.4.1. The set of quasi-randomly minimized elements is defined as the set $\mathcal{Q}_{\mathrm{qr}}$ of 0-flag algebra elements $f \in \mathcal{A}^{0}$ such that $\phi_{\mathrm{qr}}$ minimizes the density of $f$, that is, we have

$$
\mathcal{Q}_{\mathrm{qr}}=\left\{f \in \mathcal{A}^{0}: \phi_{\mathrm{qr}}(f)=\min \left\{\phi(f): \phi \in \operatorname{Hom}^{+}\left(\mathcal{A}^{0}, \mathbb{R}\right)\right\}\right\} .
$$

The set of minimization quasi-random characterizers is defined as the set $\mathcal{P}_{\mathrm{qr}}$ of 0 -flag algebra elements $f \in \mathcal{A}^{0}$ such that $\phi$ minimizes the density of $f$ if and only if $\phi=\phi_{\mathrm{qr}}$, that is, we have

$$
\begin{aligned}
\mathcal{P}_{\mathrm{qr}} & =\left\{f \in \mathcal{A}^{0}: \forall \phi \in \operatorname{Hom}^{+}\left(\mathcal{A}^{0}, \mathbb{R}\right),\left(\phi(f)=\min \left\{\psi(f): \psi \in \operatorname{Hom}^{+}\left(\mathcal{A}^{0}, \mathbb{R}\right)\right\} \Leftrightarrow \phi=\phi_{\mathrm{qr}}\right)\right\} \\
& =\left\{f \in \mathcal{Q}_{\mathrm{qr}}: \forall \phi \in \operatorname{Hom}^{+}\left(\mathcal{A}^{0}, \mathbb{R}\right),\left(\phi(f)=\phi_{\mathrm{qr}}(f) \Longrightarrow \phi=\phi_{\mathrm{qr}}\right)\right\} .
\end{aligned}
$$

It follows from definition that $\mathcal{P}_{\text {qr }}$ is a subset of $\mathcal{Q}_{\text {qr }}$ and it is easy to see that $0,1_{0} \in \mathcal{Q}_{\mathrm{qr}}$. Furthermore, from Theorem 2.2.2, we already know that $\operatorname{Tr}_{3} \in \mathcal{Q}_{\mathrm{qr}}$ and $\operatorname{Tr}_{k} \in \mathcal{P}_{\mathrm{qr}}$ for every $k \geqslant 4$. Moreover, Property $P_{2}$ says that $\operatorname{Tr}_{4}+R_{4} \in \mathcal{P}_{\mathrm{qr}}$.

We now list some trivial ways of obtaining elements of these sets from other known elements of these sets. 
Proposition 2.4.2. Suppose $f \in \mathcal{P}_{\mathrm{qr}}$ and $g_{1}, g_{2} \in \mathcal{Q}_{\mathrm{qr}}$ are 0 -flag algebra elements, $a>0$ and $b_{1}, b_{2} \geqslant 0$ are real numbers and $k \in \mathbb{N} \backslash\{0\}$ is a positive integer. Under these circumstances, the following hold.

a. $b_{1} g_{1}+b_{2} g_{2} \in \mathcal{Q}_{\mathrm{qr}}$;

b. If $\phi_{\mathrm{qr}}\left(g_{1}\right) \geqslant 0$, then $g_{1}^{k} \in \mathcal{Q}_{\mathrm{qr}}$;

c. $a f+b_{1} g_{1} \in \mathcal{P}_{\mathrm{qr}}$;

d. If $\phi_{\mathrm{qr}}(f) \geqslant 0$, then $f^{k} \in \mathcal{P}_{\mathrm{qr}}$;

Proof. For item (a), note that since $b_{1}, b_{2} \geqslant 0$ and $g_{1}, g_{2} \in \mathcal{Q}_{\text {qr }}$, we have

$$
\phi\left(b_{1} g_{1}+b_{2} g_{2}\right)=b_{1} \phi\left(g_{1}\right)+b_{2} \phi\left(g_{2}\right) \geqslant b_{1} \phi_{\mathrm{qr}}\left(g_{1}\right)+b_{2} \phi_{\mathrm{qr}}\left(g_{2}\right)=\phi_{\mathrm{qr}}\left(b_{1} g_{1}+b_{2} g_{2}\right),
$$

for every $\phi \in \operatorname{Hom}^{+}\left(\mathcal{A}^{0}, \mathbb{R}\right)$, hence $b_{1} g_{1}+b_{2} g_{2} \in \mathcal{Q}_{\mathrm{qr}}$.

For item (b), first note that since $k$ is a positive integer, we have that $g_{1}^{k} \in \mathcal{A}^{0}$. Furthermore, since $\phi_{\mathrm{qr}}\left(g_{1}\right) \geqslant 0$, we have

$$
\phi\left(g_{1}^{k}\right)=\phi\left(g_{1}\right)^{k} \geqslant \phi_{\mathrm{qr}}\left(g_{1}\right)^{k}=\phi_{\mathrm{qr}}\left(g_{1}^{k}\right)
$$

for every $\phi \in \operatorname{Hom}^{+}\left(\mathcal{A}^{0}, \mathbb{R}\right)$, hence $g_{1}^{k} \in \mathcal{Q}_{\mathrm{qr}}$.

For item (c), since we already proved item (a), it is enough to prove that $\phi(a f+$ $\left.b_{1} g_{1}\right)=\phi_{\mathrm{qr}}\left(a f+b_{1} g_{1}\right)$ implies $\phi=\phi_{\mathrm{qr}}$. But since $a>0, b \geqslant 0, \phi(f)-\phi_{\mathrm{qr}}(f) \geqslant 0$ and $\phi\left(g_{1}\right)-\phi_{\mathrm{qr}}\left(g_{1}\right) \geqslant 0$, we have that $\phi\left(a f+b_{1} g_{1}\right)=\phi_{\mathrm{qr}}\left(a f+b_{1} g_{1}\right)$ implies $\phi(f)=\phi_{\mathrm{qr}}(f)$, hence $\phi=\phi_{\mathrm{qr}}$ (since $\left.f \in \mathcal{P}_{\mathrm{qr}}\right)$.

Item (b) follows analogously: if $\phi\left(f^{k}\right)=\phi_{\mathrm{qr}}\left(f^{k}\right)$, then we have $\phi(f)=\phi_{\mathrm{qr}}(f)$, hence $\phi=$ $\phi_{\mathrm{qr}}$ (since $f \in \mathcal{P}_{\mathrm{qr}}$ ).

We now define five operations in the Theory of Tournaments.

Definition 2.4.3. Let $T$ be a tournament. We define the tournament $\tau^{\alpha}(T)$ as the tournament obtained from $T$ by adding a vertex that beats every vertex of $T$.

Analogously, we define the tournament $\tau^{\beta}(T)$ as the tournament obtained from $T$ by adding a vertex that is beaten by every vertex of $T$.

We define the tournament $\tau^{O^{A}}(T)$ as the tournament obtained from $T$ by adding two vertices $v$ and $w$ that beat every vertex of $T$ (and $v$ beats $w$ ).

Analogously, we define the tournament $\tau^{I^{A}}(T)$ as the tournament obtained from $T$ by adding two vertices $v$ and $w$ that are beaten by every vertex of $T$ (and $v$ beats $w$ ).

Finally, we define the tournament $\tau^{\operatorname{Tr}_{3}^{A}}(T)$ as the tournament obtained from $T$ by adding two vertices $v$ and $w$ such that $v$ beats every other vertex (including $w$ ) and $w$ is beaten by every other vertex.

We extend these operations linearly to $\mathbb{R} \mathcal{F}^{0}$.

Remark 2.4.4. Note that the image of the 0 -kernel $\mathcal{K}^{0}$ by these operations is not contained in $\mathcal{K}^{0}$. This can be seen through the following simple counter-examples.

$$
\begin{aligned}
\tau^{\alpha}\left(\operatorname{Tr}_{1}-\operatorname{Tr}_{2}\right) & =\tau^{\beta}\left(\operatorname{Tr}_{1}-\operatorname{Tr}_{2}\right) \\
=\tau^{O^{A}}\left(\operatorname{Tr}_{0}-\operatorname{Tr}_{1}\right)=\tau^{I^{A}}\left(\operatorname{Tr}_{0}-\operatorname{Tr}_{1}\right) & =\tau^{\operatorname{Tr}_{3}^{A}}\left(\operatorname{Tr}_{0}-\operatorname{Tr}_{1}\right) \\
& =\operatorname{Tr}_{2}-\operatorname{Tr}_{3} \notin \mathcal{K}^{0} .
\end{aligned}
$$

This means that these operations do not induce operations $\mathcal{A}^{0} \rightarrow \mathcal{A}^{0}$. 
The main theorem of this section will give us a non-trivial way of obtaining elements of $\mathcal{P}_{\text {qr }}$ from elements of $\mathcal{Q}_{\text {qr }}$ through the last three operations. But to prove it, we need two basic properties of these operations.

Lemma 2.4.5. If $T$ is a tournament, then

$$
\begin{aligned}
|\operatorname{Aut}(T)| & =\left|\operatorname{Aut}\left(\tau^{\alpha}(T)\right)\right|=\left|\operatorname{Aut}\left(\tau^{\beta}(T)\right)\right| \\
& =\left|\operatorname{Aut}\left(\tau^{O^{A}}(T)\right)\right|=\left|\operatorname{Aut}\left(\tau^{I^{A}}(T)\right)\right|=\left|\operatorname{Aut}\left(\tau^{\operatorname{Tr}_{3}^{A}}(T)\right)\right| .
\end{aligned}
$$

Proof. Let $v$ be the vertex added to $T$ in the operation $\tau^{\alpha}$. Note that $v$ is the only vertex of $\tau^{\alpha}(T)$ with indegree 0 , this implies that every automorphism of $\tau^{\alpha}(T)$ must fix $v$, which in turn implies that $f \in \operatorname{Aut}\left(\tau^{\alpha}(T)\right)$ if and only if $\left.f\right|_{V(T)} \in \operatorname{Aut}(T)$. Therefore $\left|\operatorname{Aut}\left(\tau^{\alpha}(T)\right)\right|=$ $|\operatorname{Aut}(T)|$.

The proof for $\tau^{\beta}$ is analogous (using outdegree instead of indegree).

Let $v$ and $w$ be the vertices added to $T$ in the operation $\tau^{O^{A}}$. Note that $v$ is the only vertex of $\tau^{O^{A}}(T)$ with indegree 0 and $w$ is the only vertex of $\tau^{O^{A}}(T)$ with indegree 1 , this implies that every automorphism of $\tau^{O^{A}}(T)$ must fix $v$ and $w$, which in turn implies that $f \in \operatorname{Aut}\left(\tau^{O^{A}}(T)\right)$ if and only if $\left.f\right|_{V(T)} \in \operatorname{Aut}(T)$. Therefore $\left|\operatorname{Aut}\left(\tau^{O^{A}}(T)\right)\right|=|\operatorname{Aut}(T)|$.

The proofs for $\tau^{I^{A}}$ and $\tau^{\operatorname{Tr}_{3}^{A}}$ are analogous (using outdegree instead of indegree for the first and noting that $v$ and $w$ are the only vertices with indegree and outdegree 0 respectively for the latter).

Lemma 2.4.6. If $T$ is a tournament of size $k$, then

$$
\begin{aligned}
\llbracket \pi^{\alpha}(T) \cdot \alpha^{k} \rrbracket_{1} & =\frac{\tau^{\alpha}(T)}{k+1} ; \\
\llbracket \pi^{\beta}(T) \cdot \beta^{k} \rrbracket_{1} & =\frac{\tau^{\beta}(T)}{k+1} ; \\
\llbracket \pi^{O^{A}}(T) \cdot\left(O^{A}\right)^{k} \rrbracket_{A} & =\frac{\tau^{O^{A}}(T)}{(k+2)(k+1)} ; \\
\llbracket \pi^{I^{A}}(T) \cdot\left(I^{A}\right)^{k} \rrbracket_{A} & =\frac{\tau^{I^{A}}(T)}{(k+2)(k+1)} ; \\
\llbracket \pi^{\operatorname{Tr}_{3}^{A}}(T) \cdot\left(\operatorname{Tr}_{3}^{A}\right)^{k} \rrbracket_{A} & =\frac{\tau^{\operatorname{Tr}_{3}^{A}}(T)}{(k+2)(k+1)}
\end{aligned}
$$

Proof. Let $F$ be the 1-flag obtained from $\tau^{\alpha}(T)$ by labelling the vertex with indegree 0 . Note that $\llbracket F \rrbracket_{1}=\tau^{\alpha}(T) /(k+1)$. The result now follows from

$$
\pi^{\alpha}(T)=\frac{F}{\alpha^{k}}
$$

The proof for $\tau^{\beta}$ is analogous.

Let $F$ be the $A$-flag obtained from $\tau^{O^{A}}(T)$ by labelling the two added vertices. Note that $\llbracket F \rrbracket_{A}=\tau^{O^{A}}(T) /((k+2)(k+1))$. The result now follows from

$$
\pi^{O^{A}}(T)=\frac{F}{\left(O^{A}\right)^{k}}
$$

The proofs for $\tau^{I^{A}}$ and $\tau^{\operatorname{Tr}_{3}^{A}}$ are analogous. 
Remark 2.4.7. Recall Subsection 1.4 .2 and note that if $f \in \mathcal{A}^{0}$ and $\phi \in \operatorname{Hom}^{+}\left(\mathcal{A}^{1}, \mathbb{R}\right)$, then

- $\phi\left(\pi^{\alpha}(f)\right)$ measures the density of $f$ in the outneighbourhood of the labelled vertex of $\phi$;

- $\phi\left(\pi^{\beta}(f)\right)$ measures the density of $f$ in the inneighbourhood of the labelled vertex of $\phi$.

Furthermore, if $\psi \in \operatorname{Hom}^{+}\left(\mathcal{A}^{A}, \mathbb{R}\right)$, then

- $\psi\left(\pi^{I^{A}}(f)\right)$ measures the density of $f$ in the common inneighbourhood of the labelled vertices of $\psi$;

- $\psi\left(\pi^{\operatorname{Tr}_{3}^{A}}(f)\right)$ measures the density of $f$ in the set of vertices that beat the vertex with label 2 and are beaten by the vertex with label 1.

The next theorem gives us a non-trivial way of obtaining elements of $\mathcal{Q}_{\text {qr }}$ from previously known ones. It is also a useful warm-up for the main theorem of this section.

Theorem 2.4.8. Let $k \geqslant 2$ be an integer and $f \in \mathbb{R} \mathcal{F}_{k}^{0}$ be a formal linear combination of tournaments of size $k$. If $f \in \mathcal{Q}_{\mathrm{qr}}$ and $\phi_{\mathrm{qr}}(f) \geqslant 0$, then $\tau^{\alpha}(f), \tau^{\beta}(f) \in \mathcal{Q}_{\mathrm{qr}}$.

Furthermore, if $\phi_{\mathrm{qr}}(f)>0$ and $\phi \in \operatorname{Hom}^{+}\left(\mathcal{A}^{0}, \mathbb{R}\right)$ is such that $\phi\left(\tau^{\alpha}(f)\right)=\phi_{\mathrm{qr}}\left(\tau^{\alpha}(f)\right)$ or $\phi\left(\tau^{\beta}(f)\right)=\phi_{\mathrm{qr}}\left(\tau^{\beta}(f)\right)$, then $\phi$ is balanced.

Proof. We will prove the result only for $\tau^{\alpha}$ since the proof for $\tau^{\beta}$ is analogous.

Write $f=\sum_{T \in \mathcal{F}_{k}^{0}} c_{T} T$, with $c_{T} \in \mathbb{R}$.

From Lemma 2.4.6, for every homomorphism $\phi \in \operatorname{Hom}^{+}\left(\mathcal{A}^{0}, \mathbb{R}\right)$, we have

$$
\begin{aligned}
\phi\left(\tau^{\alpha}(f)\right) & =\sum_{T \in \mathcal{F}_{k}^{0}} c_{T} \phi\left(\tau^{\alpha}(T)\right) \\
& =\sum_{T \in \mathcal{F}_{k}^{0}} c_{T}(k+1) \phi\left(\llbracket \pi^{\alpha}(T) \cdot \alpha^{k} \rrbracket_{1}\right) \\
& =\sum_{T \in \mathcal{F}_{k}^{0}} c_{T}(k+1) \mathbb{E}\left[\boldsymbol{\phi}^{\mathbf{1}}\left(\pi^{\alpha}(T)\right) \cdot \boldsymbol{\phi}^{\mathbf{1}}(\alpha)^{k}\right] \\
& =(k+1) \mathbb{E}\left[\boldsymbol{\phi}^{\mathbf{1}}\left(\pi^{\alpha}(f)\right) \cdot \boldsymbol{\phi}^{\mathbf{1}}(\alpha)^{k}\right] .
\end{aligned}
$$

Since $\boldsymbol{\phi}^{\mathbf{1}}(\alpha)>0$ implies that $\boldsymbol{\phi}^{\mathbf{1}} \circ \pi^{\alpha} \in \operatorname{Hom}^{+}\left(\mathcal{A}^{0}, \mathbb{R}\right)$ and since $f \in \mathcal{Q}_{\mathrm{qr}}$, we have

$$
\mathbb{E}\left[\phi^{\mathbf{1}}\left(\pi^{\alpha}(f)\right) \cdot \boldsymbol{\phi}^{\mathbf{1}}(\alpha)^{k}\right] \geqslant \phi_{\mathrm{qr}}(f) \mathbb{E}\left[\boldsymbol{\phi}^{\mathbf{1}}(\alpha)^{k}\right] .
$$

Now, by Jensen's inequality, we have

$$
\mathbb{E}\left[\boldsymbol{\phi}^{\mathbf{1}}(\alpha)^{k}\right] \geqslant \mathbb{E}\left[\boldsymbol{\phi}^{\mathbf{1}}(\alpha)\right]^{k}=\phi\left(\llbracket \alpha \rrbracket_{1}\right)^{k}=\frac{1}{2^{k}} .
$$

Note now that for every $T \in \mathcal{F}_{k}^{0}$, Lemma 2.4.5 gives

$$
\phi_{\mathrm{qr}}(T)=\frac{k !}{|\operatorname{Aut}(T)| 2^{\left(\begin{array}{c}
k \\
2
\end{array}\right)}}=\frac{(k+1) !}{\left|\operatorname{Aut}\left(\tau^{\alpha}(T)\right)\right| 2^{\left(\begin{array}{c}
k+1 \\
2
\end{array}\right)}} \cdot \frac{2^{k}}{k+1}=\frac{2^{k}}{k+1} \phi_{\mathrm{qr}}\left(\tau^{\alpha}(T)\right),
$$

which means that

$$
\phi_{\mathrm{qr}}(f)=\frac{2^{k}}{k+1} \phi_{\mathrm{qr}}\left(\tau^{\alpha}(f)\right)
$$


Gathering everything, since $\phi_{\mathrm{qr}}(f) \geqslant 0$, we get

$$
\phi\left(\tau^{\alpha}(f)\right) \geqslant(k+1) \phi_{\mathrm{qr}}(f) \mathbb{E}\left[\phi^{\mathbf{1}}(\alpha)^{k}\right] \geqslant \frac{k+1}{2^{k}} \phi_{\mathrm{qr}}(f)=\phi_{\mathrm{qr}}\left(\tau^{\alpha}(f)\right) .
$$

Therefore $\tau^{\alpha}(f) \in \mathcal{Q}_{\mathrm{qr}}$. Note now that if $\phi\left(\tau^{\alpha}(f)\right)=\phi_{\mathrm{qr}}\left(\tau^{\alpha}(f)\right)$, then we must have equality throughout the proof. In particular, since we used Jensen's inequality in (2.4) with the strictly convex function $x \mapsto x^{k}$ (for $x \geqslant 0$ ), if $\phi_{\mathrm{qr}}(f)>0$, then $\phi^{\mathbf{1}}(\alpha)$ is almost surely a constant $C$. But we have

$$
C=\mathbb{E}\left[\phi^{\mathbf{1}}(\alpha)\right]=\phi\left(\llbracket \alpha \rrbracket_{1}\right)=\frac{1}{2}
$$

therefore $\phi$ is balanced.

We can now prove the main theorem of this section. The idea of its proof is very similar to the one in Theorem 2.4.8.

Theorem 2.4.9. Let $k \geqslant 2$ be an integer and $f \in \mathbb{R} \mathcal{F}_{k}^{0}$ be a formal linear combination of tournaments of size $k$. If $f \in \mathcal{Q}_{\mathrm{qr}}$ and $\phi_{\mathrm{qr}}(f)>0$, then $\tau^{O^{A}}(f), \tau^{I^{A}}(f), \tau^{\operatorname{Tr}_{3}^{A}} \in \mathcal{P}_{\mathrm{qr}}$.

Proof. Write $f=\sum_{T \in \mathcal{F}_{k}^{0}} c_{T} T$, with $c_{T} \in \mathbb{R}$.

Since $\llbracket 1_{A} \rrbracket_{A}=1 / 2$, from Lemma 2.4.6, for every homomorphism $\phi \in \operatorname{Hom}^{+}\left(\mathcal{A}^{0}, \mathbb{R}\right)$, we have

$$
\begin{aligned}
\phi\left(\tau^{O^{A}}(f)\right) & =\sum_{T \in \mathcal{F}_{k}^{0}} c_{T} \phi\left(\tau^{O^{A}}(T)\right) \\
& =\sum_{T \in \mathcal{F}_{k}^{0}} c_{T}(k+2)(k+1) \phi\left(\llbracket \pi^{O^{A}}(T) \cdot\left(O^{A}\right)^{k} \rrbracket_{A}\right) \\
& =\sum_{T \in \mathcal{F}_{k}^{0}} c_{T} \frac{(k+2)(k+1)}{2} \mathbb{E}\left[\boldsymbol{\phi}^{\boldsymbol{A}}\left(\pi^{O^{A}}(T)\right) \cdot \boldsymbol{\phi}^{\boldsymbol{A}}\left(O^{A}\right)^{k}\right] \\
& =\frac{(k+2)(k+1)}{2} \mathbb{E}\left[\boldsymbol{\phi}^{\boldsymbol{A}}\left(\pi^{O^{A}}(f)\right) \cdot \boldsymbol{\phi}^{\boldsymbol{A}}\left(O^{A}\right)^{k}\right] .
\end{aligned}
$$

Since $\phi^{\boldsymbol{A}}\left(O^{A}\right)>0$ implies that $\boldsymbol{\phi}^{\boldsymbol{A}} \circ \pi^{O^{A}} \in \operatorname{Hom}^{+}\left(\mathcal{A}^{0}, \mathbb{R}\right)$ and since $f \in \mathcal{Q}_{\mathrm{qr}}$, we have

$$
\mathbb{E}\left[\boldsymbol{\phi}^{\boldsymbol{A}}\left(\pi^{O^{A}}(f)\right) \cdot \boldsymbol{\phi}^{\boldsymbol{A}}\left(O^{A}\right)^{k}\right] \geqslant \phi_{\mathrm{qr}}(f) \mathbb{E}\left[\boldsymbol{\phi}^{\boldsymbol{A}}\left(O^{A}\right)^{k}\right]
$$

Now, by Jensen's inequality, we have

$$
\mathbb{E}\left[\phi^{\boldsymbol{A}}\left(O^{A}\right)^{k}\right] \geqslant \mathbb{E}\left[\boldsymbol{\phi}^{\boldsymbol{A}}\left(O^{A}\right)\right]^{k}=\left(2 \phi\left(\llbracket O^{A} \rrbracket_{A}\right)\right)^{k}=\left(\frac{\phi\left(\operatorname{Tr}_{3}\right)}{3}\right)^{k} .
$$

Note now that for every $T \in \mathcal{F}_{k}^{0}$, Lemma 2.4.5 gives

$$
\begin{aligned}
\phi_{\mathrm{qr}}(T) & =\frac{k !}{|\operatorname{Aut}(T)| 2^{\left(\begin{array}{c}
k \\
2
\end{array}\right)}}=\frac{(k+2) !}{\left|\operatorname{Aut}\left(\tau^{O^{A}}(T)\right)\right| 2^{\left(\begin{array}{c}
k+2 \\
2
\end{array}\right)}} \cdot \frac{2^{2 k+1}}{(k+2)(k+1)} \\
& =\frac{2^{2 k+1}}{(k+2)(k+1)} \phi_{\mathrm{qr}}\left(\tau^{O^{A}}(T)\right),
\end{aligned}
$$


which means that

$$
\phi_{\mathrm{qr}}(f)=\frac{2^{2 k+1}}{(k+2)(k+1)} \phi_{\mathrm{qr}}\left(\tau^{O^{A}}(f)\right) .
$$

Gathering everything, since $\phi_{\mathrm{qr}}(f)>0$, we get

$$
\begin{aligned}
\phi\left(\tau^{O^{A}}(f)\right) & \geqslant \frac{(k+2)(k+1)}{2} \phi_{\mathrm{qr}}(f) \mathbb{E}\left[\phi^{\boldsymbol{A}}\left(O^{A}\right)^{k}\right] \\
& \geqslant \frac{(k+2)(k+1)}{2} \phi_{\mathrm{qr}}(f)\left(\frac{\phi\left(\operatorname{Tr}_{3}\right)}{3}\right)^{k} \\
& \geqslant \frac{(k+2)(k+1)}{2^{2 k+1}} \phi_{\mathrm{qr}}(f) \\
& =\phi_{\mathrm{qr}}\left(\tau^{O^{A}}(f)\right),
\end{aligned}
$$

where the last inequality follows from Theorem 2.2.2 and the fact that $\phi_{\mathrm{qr}}(f)>0$.

Therefore $\tau^{O^{A}}(f) \in \mathcal{Q}_{\mathrm{qr}}$. Note now that if $\phi\left(\tau^{O^{A}}(f)\right)=\phi_{\mathrm{qr}}\left(\tau^{O^{A}}(f)\right)$, then we must have equality throughout the proof. In particular, since we used Jensen's inequality in (2.5) with the strictly convex function $x \mapsto x^{k}$ (for $x \geqslant 0$ ) and $\phi_{\mathrm{qr}}(f)>0$, we have that $\boldsymbol{\phi}^{\boldsymbol{A}}\left(O^{A}\right)$ is almost surely a constant, which implies $\phi=\phi_{\text {qr }}$ by Corollary 2.3.6.

Therefore $\tau^{O^{A}}(f) \in \mathcal{P}_{\mathrm{qr}}$.

The proofs for $\tau^{I^{A}}$ and $\tau^{\operatorname{Tr}_{3}^{A}}$ are analogous.

Using $P_{2}$ and the above theorem we get, for instance, the following non-trivial elements of $\mathcal{P}_{\mathrm{qr}}$.

$$
\operatorname{Tr}_{6}+\tau^{O^{A}}\left(R_{4}\right) ; \quad \operatorname{Tr}_{6}+\tau^{I^{A}}\left(R_{4}\right) ; \quad \operatorname{Tr}_{6}+\tau^{\operatorname{Tr}_{3}^{A}}\left(R_{4}\right) .
$$

We state now some simple elements of $\mathcal{P}_{\text {qr }}$ that can be obtained with Theorems 2.4.8 and 2.4.9 together.

Corollary 2.4.10. Let $k, \ell_{1}, \ell_{2} \in \mathbb{N}$ be natural numbers such that $2 \leqslant \ell_{1}+\ell_{2} \leqslant k$ and $k-$ $\ell_{1}-\ell_{2} \geqslant 2$. Let also $\mathcal{T} \subset \mathcal{F}_{k}^{0}$ be the family of all tournaments $T$ of size $k$ such that there exist vertices $v_{0}, v_{1}, \ldots, v_{\ell_{1}-1}, w_{0}, w_{1}, \ldots, w_{\ell_{2}-1} \in V(T)$ with the indegree of each $v_{i}$ being $i$ and the outdegree of each $w_{i}$ being $i$.

Under these circumstances, if $f=\sum_{T \in \mathcal{T}} T$, then $f \in \mathcal{P}_{\mathrm{qr}}$.

Proof. The result follows directly from Theorems 2.4 .8 and 2.4.9, from the observation that $\sum_{T \in \mathcal{F}_{k-\ell_{1}-\ell_{2}}^{0}} T=1_{0} \in \mathcal{Q}_{\mathrm{qr}}$, and by noting the following three cases.

If $\ell_{1} \geqslant 2$, then

$$
f=\tau^{O^{A}} \circ \underbrace{\tau^{\alpha} \circ \tau^{\alpha} \circ \cdots \tau^{\alpha}}_{\ell_{1}-2 \text { times }} \circ \underbrace{\tau^{\beta} \circ \tau^{\beta} \circ \cdots \tau^{\beta}}_{\ell_{2} \text { times }}\left(\sum_{T \in \mathcal{F}_{k-\ell_{1}-\ell_{2}}^{0}} T\right) .
$$

If $\ell_{2} \geqslant 2$, then

$$
f=\tau^{I^{A}} \circ \underbrace{\tau^{\beta} \circ \tau^{\beta} \circ \cdots \tau^{\beta}}_{\ell_{2}-2 \text { times }} \circ \underbrace{\tau^{\alpha} \circ \tau^{\alpha} \circ \cdots \tau^{\alpha}}_{\ell_{1} \text { times }}\left(\sum_{T \in \mathcal{F}_{k-\ell_{1}-\ell_{2}}^{0}} T\right) .
$$


If $\ell_{1}=\ell_{2}=1$, then

$$
f=\tau^{\operatorname{Tr}_{3}^{A}}\left(\sum_{T \in \mathcal{F}_{k-\ell_{1}-\ell_{2}}^{0}} T\right)
$$




\section{Chapter 3}

\section{Carousel tournaments and quasi-carouselness}

In this chapter we will present the quasi-carousel properties, which are, in the same fashion as the quasi-random properties, a set of equivalent characterizations of one special homomorphism $\phi_{\mathrm{R}}$, called carousel homomorphism, in the Theory of Tournaments.

We will start by presenting in Section 3.1 some basic results concerning locally transitive tournaments.

In Section 3.2, we will present the carousel homomorphism as the limit of the sequence of carousel tournaments (Lemma 3.2.1) and we will state the quasi-carousel properties and their equivalence (Theorem 3.2.2) comparing them with quasi-random properties and several of these comparisons will suggest that the homomorphisms $\phi_{\mathrm{qr}}$ and $\phi_{\mathrm{R}}$ are the furthest possible from each other within the class of balanced homomorphisms. The proofs of Lemma 3.2.1 and Theorem 3.2.2, however, will only be presented in Section 3.3.

Throughout this chapter, unless explicitly stated otherwise, all flag algebra terms and symbols will be relative to the theory $T_{\text {Tournaments }}$.

\subsection{Locally transitive tournaments}

In this section we will present some basic facts about locally transitive tournaments.

Definition 3.1.1. A tournament $T$ is locally transitive if for every vertex $v \in V(T)$, the outneighbourhood $N^{+}(v)=\{w \in V(T): v w \in A(T)\}$ and the inneighbourhood $N^{-}(v)=$ $\{w \in V(T): w v \in A(T)\}$ of $v$ are both transitive.

The following alternative characterization easily follows from the definition.

Proposition 3.1.2. A tournament $T$ is locally transitive if and only if it has no copy of $W_{4}$ nor of $L_{4}$, that is, we have $p\left(W_{4}+L_{4} ; T\right)=0$.

This proposition motivates the following definition on the flag algebra of tournaments.

Definition 3.1.3. A homomorphism $\phi$ in the Theory of Tournaments is locally transitive if $\phi\left(W_{4}+L_{4}\right)=0$.

Remark 3.1.4. Note that the fact that a tournament sequence $\left(T_{n}\right)_{n \in \mathbb{N}}$ converges to a locally transitive homomorphism does not imply that any of the tournaments $T_{n}$ is locally transitive; it only implies that the sequence is almost locally transitive in the sense that the densities of $W_{4}$ and $L_{4}$ tend to zero.

Note that if $v$ is a vertex of a locally transitive tournament $T$, then the $\operatorname{arcs}$ of $T$ induce linear orders on the sets $N^{+}(v)$ and $N^{-}(v)$ (that is, defining $w<_{T} z \Longleftrightarrow w z \in A(T)$, 
the restriction of the relation $<_{T}$ to either of these sets induces a linear order). With this observation, Brouwer obtained the following properties of locally transitive tournaments.

Proposition 3.1.5 (Brouwer [Bro80]). If $v$ is a vertex of a locally transitive tournament $T$ and $a \in N^{+}(v)$ is a vertex in the outneighbourhood of $v$, then $N^{+}(a)$ is the union of a terminal interval of $N^{+}(v)$ and an initial interval of $N^{-}(v)$ (in the order induced by the arcs of $T$ in these sets).

Proposition 3.1.6 (Brouwer [Bro80]). A tournament $T$ is locally transitive if and only if it can be cyclically ordered in a way such that for every vertex $v \in V(T)$, the set $N^{+}(v) \cup\{v\}$ is an interval of the cyclic order with initial endpoint $v$.

Recall that a balanced tournament is a tournament of odd order $2 n+1$ such that every vertex has outdegree $n$. With this definition, it is easy to see the following corollary.

Corollary 3.1.7. For every $n \in \mathbb{N}$, there is exactly one up to isomorphism balanced locally transitive tournament of order $2 n+1$. This tournament is called carousel tournament of order $2 n+1$, is denoted by $R_{2 n+1}$, and is given by

$$
V\left(R_{2 n+1}\right)=\{0,1, \ldots, 2 n\} ; \quad A\left(R_{2 n+1}\right)=\{(x,(x+i) \bmod (2 n+1)): i \in[n]\} .
$$

\subsection{The carousel homomorphism and the quasi-carou- sel properties}

As expected, we define the carousel homomorphism as the limit of the sequence of carousel tournaments.

Lemma 3.2.1. The sequence of tournaments $\left(R_{2 n+1}\right)_{n \in \mathbb{N}}$ is convergent and its limit is called carousel homomorphism and is denoted by $\phi_{\mathrm{R}}$.

We will now list the quasi-carousel properties, which are properties of a homomorphism $\phi \in$ $\operatorname{Hom}^{+}\left(\mathcal{A}^{0}, \mathbb{R}\right)$ that we will prove to hold if and only if $\phi=\phi_{\mathrm{R}}$. Property $S_{1}$ is stated just for practical reasons and the equivalence of Properties $S_{1}$ and $S_{2}$ implies that $\phi_{\mathrm{R}}$ is the only homomorphism that is both balanced and locally transitive.

$S_{1}: \phi=\phi_{\mathrm{R}}$

$S_{2}: \phi$ is balanced and locally transitive;

$S_{3}$ : $\phi$ maximizes the density of $R_{4}$, i.e., we have

$$
\phi\left(R_{4}\right)=\max \left\{\psi\left(R_{4}\right): \psi \in \operatorname{Hom}^{+}\left(\mathcal{A}^{0}, \mathbb{R}\right)\right\} ;
$$

$S_{4}: \phi$ maximizes the second moment of $\phi^{\boldsymbol{A}}\left(\vec{C}_{3}^{A}\right)$.

It will be more practical to state the next quasi-carousel properties with free parameters $F$ and $q$, which will be respectively an $A$-flag algebra element and a real number.

$S_{5}(F, q): \boldsymbol{\phi}^{\boldsymbol{A}}(F) \sim U(0, q)$ (that is, the random variable $\boldsymbol{\phi}^{\boldsymbol{A}}(F)$ is uniformly distributed in the interval $[0, q])$;

$S_{6}(F, q): \phi$ maximizes the second moment of $\phi^{A}(F)$ restricted to $\mathbb{E}\left[\phi^{A}(F)\right]=q$, i.e., we have $\mathbb{E}\left[\boldsymbol{\phi}^{\boldsymbol{A}}(F)\right]=q$ and

$$
\mathbb{E}\left[\boldsymbol{\phi}^{\boldsymbol{A}}(F)^{2}\right]=\max \left\{\mathbb{E}\left[\boldsymbol{\psi}^{\boldsymbol{A}}(F)^{2}\right]: \psi \in \operatorname{Hom}^{+}\left(\mathcal{A}^{0}, \mathbb{R}\right) \wedge \mathbb{E}\left[\boldsymbol{\psi}^{\boldsymbol{A}}(F)\right]=q\right\}
$$


We can now state the main theorem on quasi-carouselness.

Theorem 3.2.2 (C. [Cor15, Theorem 3.1]). If $F \in \mathcal{F}_{3}^{A}$ is an $A$-flag of size 3 and $G$ is either $O^{A}+I^{A}$ or $\vec{C}_{3}^{A}+\operatorname{Tr}_{3}^{A}$, then

$$
S_{1} \Rightarrow S_{2} \Rightarrow S_{3} \Rightarrow S_{4} \Rightarrow S_{5}(F, 1 / 2) \Rightarrow S_{6}(F, 1 / 4) \Rightarrow S_{5}(G, 1) \Rightarrow S_{6}(G, 1 / 2) \Rightarrow S_{1} .
$$

The rest of this section is devoted to comparing the quasi-carousel properties with the quasi-random properties (see Table 3.1 for a summary). Each comparison we provide will suggest that within the class of balanced homomorphisms, the homomorphisms $\phi_{\mathrm{qr}}$ and $\phi_{\mathrm{R}}$ are the furthest possible from each other.

Note that quasi-carousel Property $S_{2}$ says that $\phi_{\mathrm{R}}$ is the only balanced locally transitive homomorphism; this means that $\phi_{\mathrm{R}}\left(W_{4}+L_{4}\right)=0$. Therefore $\phi_{\mathrm{R}}$ maximizes the density of $\operatorname{Tr}_{4}+R_{4}$ within the class of balanced homomorphisms as opposed to quasi-random Property $P_{2}$, which says that $\phi_{\mathrm{qr}}$ minimizes the density of $\operatorname{Tr}_{4}+R_{4}$.

Recall now from Lemma 2.3.5 that $\phi \in \operatorname{Hom}^{+}\left(\mathcal{A}^{0}, \mathbb{R}\right)$ is balanced if and only if $\phi\left(\operatorname{Tr}_{4}\right)=$ $\phi\left(R_{4}\right)$, hence quasi-carousel Property $S_{2}$ also implies that $\phi_{\mathrm{R}}$ maximizes the density of $\operatorname{Tr}_{4}$ within the class of balanced homomorphisms. This opposes the quasi-random property from Theorem 2.2.2 that says that $\phi_{\mathrm{qr}}$ minimizes the density of $\operatorname{Tr}_{4}$.

On the other hand, Lemma 2.3.5 also implies that $\phi_{\mathrm{qr}}$ minimizes the density of $R_{4}$ within the class of balanced homomorphisms. This opposes quasi-carousel Property $S_{3}$.

Finally, it is easy to see (see proof of Lemma 3.3.6) that the conditions on the expected value of quasi-carousel properties $S_{6}(F, 1 / 4)\left(F \in \mathcal{F}_{3}^{A}\right)$ and $S_{6}(G, 1 / 2)\left(G \in\left\{O^{A}+I^{A}, \operatorname{Tr}_{3}^{A}+\vec{C}_{3}^{A}\right\}\right)$ are equivalent to $\phi$ being balanced. This implies that, within the class of balanced homomorphisms, the carousel homomorphism $\phi_{\mathrm{R}}$ maximizes the variance of the random variables

$$
\phi^{\boldsymbol{A}}\left(O^{A}\right), \phi^{\boldsymbol{A}}\left(I^{A}\right), \boldsymbol{\phi}^{\boldsymbol{A}}\left(\operatorname{Tr}_{3}^{A}\right), \boldsymbol{\phi}^{\boldsymbol{A}}\left(\vec{C}_{3}^{A}\right), \boldsymbol{\phi}^{\boldsymbol{A}}\left(O^{A}+I^{A}\right), \phi^{\boldsymbol{A}}\left(\operatorname{Tr}_{3}^{A}+\vec{C}_{3}^{A}\right)
$$

which opposes quasi-random Properties $P_{4}, P_{4}^{\prime}, P_{3}, P_{3}^{\prime}$ and those of Theorem 2.3.1 (or those of Corollaries 2.3.6 and 2.3.7). 
Quasi-random property

Quasi-carousel property $\phi_{\text {qr }}$ minimizes $\phi\left(\operatorname{Tr}_{4}+R_{4}\right)\left(P_{2}\right)$ $\phi_{\mathrm{qr}}$ minimizes $\phi\left(\operatorname{Tr}_{4}\right)(2.2 .2)$

$\phi_{\mathrm{qr}}$ minimizes $\phi\left(R_{4}\right)$ given $\phi$ balanced (2.2.2)

$\phi_{\mathrm{qr}}$ minimizes $\operatorname{Var}\left[\phi^{\boldsymbol{A}}\left(O^{A}\right)\right](2.3 .6)$

$\phi_{\mathrm{qr}}$ minimizes $\operatorname{Var}\left[\boldsymbol{\phi}^{\boldsymbol{A}}\left(I^{A}\right)\right](2.3 .6)$

$\phi_{\mathrm{qr}}$ minimizes $\operatorname{Var}\left[\boldsymbol{\phi}^{\boldsymbol{A}}\left(\operatorname{Tr}_{3}^{A}\right)\right](2.3 .6)$

$\phi_{\mathrm{qr}}$ minimizes $\operatorname{Var}\left[\boldsymbol{\phi}^{\boldsymbol{A}}\left(\vec{C}_{3}^{A}\right)\right]$ given $\phi$ balanced (2.3.6) $\phi_{\mathrm{qr}}$ minimizes $\operatorname{Var}\left[\boldsymbol{\phi}^{\boldsymbol{A}}\left(O^{A}+I^{A}\right)\right]$ given $\phi$ balanced $\left(P_{3}\right)$ $\phi_{\mathrm{qr}}$ minimizes $\operatorname{Var}\left[\boldsymbol{\phi}^{\boldsymbol{A}}\left(\operatorname{Tr}_{3}^{A}+\vec{C}_{3}^{A}\right)\right]$ given $\phi$ balanced $\left(P_{3}^{\prime}\right)$ $\phi_{\mathrm{R}}$ maximizes $\phi\left(\mathrm{Tr}_{4}+R_{4}\right)$ given $\phi$ balanced $\left(S_{2}\right)$ $\phi_{\mathrm{R}}$ maximizes $\phi\left(\operatorname{Tr}_{4}\right)$ given $\phi$ balanced $\left(S_{2}\right)$ $\phi_{\mathrm{R}}$ maximizes $\phi\left(R_{4}\right)\left(S_{3}\right)$ $\phi_{\mathrm{qr}}$ maximizes $\operatorname{Var}\left[\phi^{\boldsymbol{A}}\left(O^{A}\right)\right]$ given $\phi$ balanced $\left(S_{6}\right)$ $\phi_{\text {qr }}$ maximizes $\operatorname{Var}\left[\phi^{\boldsymbol{A}}\left(I^{A}\right)\right]$ given $\phi$ balanced $\left(S_{6}\right)$ $\phi_{\text {qr }}$ maximizes $\operatorname{Var}\left[\boldsymbol{\phi}^{\boldsymbol{A}}\left(\operatorname{Tr}_{3}^{A}\right)\right]$ given $\phi$ balanced $\left(S_{6}\right)$ $\phi_{\mathrm{qr}}$ maximizes $\operatorname{Var}\left[\boldsymbol{\phi}^{\boldsymbol{A}}\left(\vec{C}_{3}^{A}\right)\right]$ given $\phi$ balanced $\left(S_{6}\right)$ $\phi_{\mathrm{qr}}$ maximizes $\operatorname{Var}\left[\boldsymbol{\phi}^{\boldsymbol{A}}\left(O^{A}+I^{A}\right)\right]$ given $\phi$ balanced $\left(S_{6}\right)$ $\phi_{\mathrm{qr}}$ maximizes $\operatorname{Var}\left[\phi^{\boldsymbol{A}}\left(\operatorname{Tr}_{3}^{A}+\vec{C}_{3}^{A}\right)\right]$ given $\phi$ balanced $\left(S_{6}\right)$

Table 3.1: Comparison of quasi-random properties and quasi-carousel properties. 


\subsection{Quasi-carouselness proofs}

We will start by proving Theorem 3.2.2 (supposing that Lemma 3.2.1 is true) through a series of lemmas, enlarging the family of properties known to be quasi-carousel properties after each lemma.

Lemma 3.3.1 (C. [Cor15, Lemma 3.2]). We have $S_{1} \Longleftrightarrow S_{2}$.

Proof. Since $R_{2 n+1}$ is a locally transitive balanced tournament for every $n \in \mathbb{N}$, it follows that $\phi_{\mathrm{R}}$ is a balanced and locally transitive homomorphism.

Suppose that $\phi \in \operatorname{Hom}^{+}\left(\mathcal{A}^{0}\left[T_{\text {Tournaments }}\right], \mathbb{R}\right)$ satisfies $S_{2}$ and let $T_{\neg\left\{W_{4}, L_{4}\right\}}$ be the theory of tournaments without any occurrence of $W_{4}$ or $L_{4}$, i.e., the theory of locally transitive tournaments (this is a universal theory, see Example 1.1.6). Note that $\phi$ is also an element of $\operatorname{Hom}^{+}\left(\mathcal{A}^{0}\left[T_{\neg\left\{W_{4}, L_{4}\right\}}\right], \mathbb{R},\right)$, hence there exists a sequence $\left(T_{n}\right)_{n \in \mathbb{N}}$ of models in $T_{\neg\left\{W_{4}, L_{4}\right\}}$ (i.e., locally transitive tournaments) converging to $\phi$ and we can take this sequence to be such that $\left|T_{n}\right|$ is odd for every $n \in \mathbb{N}$.

Since $\phi$ is balanced, we know that all but $o\left(\left|T_{n}\right|\right)$ vertices of $T_{n}$ have outdegree $(1 / 2+$ $o(1))\left|T_{n}\right|$ hence, considering the cyclic ordering of $T_{n}$ given by Proposition 3.1.6, we see that we can obtain $R_{\left|T_{n}\right|}$ from $T_{n}$ by flipping $o\left(\left|T_{n}\right|^{2}\right)$ arcs of $T_{n}$. Since this flipping operation does not change the limit homomorphism, we have that $\left(T_{n}\right)_{n \in \mathbb{N}}$ converges to the same limit as a subsequence of $\left(R_{2 n+1}\right)_{n \in \mathbb{N}}$. Therefore, we have $\phi=\phi_{\mathrm{R}}$.

Lemma 3.3.2 (C. [Cor15, Lemma 3.3]). We have $S_{1} \Longleftrightarrow S_{3}$.

Proof. Note first that Lemma 2.3 .5 immediately gives that $\phi\left(R_{4}\right) \leqslant 1 / 2$ for every $\phi \in$ $\operatorname{Hom}^{+}\left(\mathcal{A}^{0}, \mathbb{R}\right)$.

We already know from Lemma 3.3.1 that $\phi_{\mathrm{R}}$ is balanced and locally transitive, hence we have $\phi_{\mathrm{R}}\left(W_{4}+L_{4}\right)=0$ and $\phi_{\mathrm{R}}\left(\operatorname{Tr}_{4}\right)=\phi_{\mathrm{R}}\left(R_{4}\right)$. This implies that $\phi_{\mathrm{R}}\left(\operatorname{Tr}_{4}+R_{4}\right)=1$, hence $\phi_{\mathrm{R}}\left(R_{4}\right)=1 / 2$, which means that $\phi_{\mathrm{R}}$ maximizes the density of $R_{4}\left(S_{3}\right)$.

Suppose now that $\phi \in \operatorname{Hom}^{+}\left(\mathcal{A}^{0}, \mathbb{R}\right)$ is a homomorphism maximizing the density of $R_{4}$, that is, we have $\phi\left(R_{4}\right)=1 / 2$.

Since $\phi\left(\operatorname{Tr}_{4}+R_{4}\right) \leqslant 1$, a double application of Lemma 2.3.5 implies that $\phi\left(\operatorname{Tr}_{4}\right)=1 / 2$ and that $\phi$ is balanced, hence $\phi$ satisfies $S_{2}$ (since $\left.\phi\left(W_{4}+L_{4}\right)=1-\phi\left(\operatorname{Tr}_{4}+R_{4}\right)\right)$. Therefore $\phi=\phi_{\mathrm{R}}$ (by Lemma 3.3.1).

The proof of the previous lemma also gives us the value of $\phi_{\mathrm{R}}\left(R_{4}\right)$ as a corollary.

Corollary 3.3.3 (C. [Cor15, Corollary 3.4]). If $\phi \in \operatorname{Hom}^{+}\left(\mathcal{A}^{0}, \mathbb{R}\right)$, then $\phi\left(R_{4}\right) \leqslant 1 / 2$ with equality if and only if $\phi=\phi_{\mathrm{R}}$.

Let us continue with the proof of Theorem 3.2.2.

Lemma 3.3.4 (C. [Cor15, Lemma 3.5]). We have $S_{1} \Longleftrightarrow S_{4}$.

Proof. Note that

$$
\mathbb{E}\left[\phi^{A}\left(\vec{C}_{3}^{A}\right)^{2}\right]=\frac{\phi\left(R_{4}\right)}{6}
$$

hence $\phi$ maximizes the second moment of $\phi^{A}\left(\vec{C}_{3}^{A}\right)$ if and only if $\phi$ maximizes the density of $R_{4}$. Therefore the result follows from Lemma 3.3.2. 
Lemma 3.3.5 (C. [Cor15]). If $F \in \mathcal{F}_{3}^{A}$ is an $A$-flag of size 3 and $G \in \mathcal{A}^{A}$ is either $O^{A}+I^{A}$ or $\operatorname{Tr}_{3}^{A}+\vec{C}_{3}^{A}$, then we have

$$
S_{1} \Longrightarrow S_{5}(F, 1 / 2) \wedge S_{5}(G, 1)
$$

Proof. Let $\mathbb{P}^{A}$ be the Borel probability measure of $\boldsymbol{\phi}_{\mathbf{R}}^{\boldsymbol{A}}$ and for every $a \leqslant b$, let

$$
\begin{aligned}
B_{a, b}(F) & =\left\{x \in[0,1]^{\mathcal{F}^{A}}: a<x_{F}<b\right\} ; \\
B_{a, b}\left(O^{A}+I^{A}\right) & =\left\{x \in[0,1]^{\mathcal{F}^{A}}: a<x_{O^{A}}+x_{I^{A}}<b\right\} ; \\
B_{a, b}\left(\operatorname{Tr}_{3}^{A}+\vec{C}_{3}^{A}\right) & =\left\{x \in[0,1]^{\mathcal{F}^{A}}: a<x_{\operatorname{Tr}_{3}^{A}}+x_{\vec{C}_{3}^{A}}<b\right\}
\end{aligned}
$$

Note that $B_{a, b}$ is an open subset of $[0,1]^{\mathcal{F}^{A}}$. Since $\left(\mathbb{P}_{R_{2 n+1}}^{A}\right)_{n \in \mathbb{N}}$ weakly converges to $\mathbb{P}^{A}$, by Theorem 1.3.10, it is enough to prove that

$$
\liminf _{n \rightarrow \infty} \mathbb{P}_{R_{2 n+1}}^{A}\left[B_{a, b}(F)\right]=2(b-a),
$$

for every $0 \leqslant a \leqslant b \leqslant 1 / 2$;

$$
\liminf _{n \rightarrow \infty} \mathbb{P}_{R_{2 n+1}}^{A}\left[B_{a, b}(F)\right]=1-2 a,
$$

for every $0 \leqslant a \leqslant 1 / 2 \leqslant b \leqslant 1$; and

$$
\liminf _{n \rightarrow \infty} \mathbb{P}_{R_{2 n+1}}^{A}\left[B_{a, b}(G)\right]=b-a,
$$

for every $0 \leqslant a \leqslant b \leqslant 1$.

Recall the definition of $\mathbb{P}_{R_{2 n+1}}^{A}$ : consider the random experiment where we pick at random an embedding $\boldsymbol{\theta}$ of $A$ in $R_{2 n+1}$, then we have

$$
\begin{aligned}
& \mathbb{P}_{R_{2 n+1}}^{A}\left[B_{a, b}(F)\right]=\mathbb{P}\left[a<p\left(F ; \boldsymbol{L}_{\mathbf{2 n + 1}}\right)<b\right], \\
& \mathbb{P}_{R_{2 n+1}}^{A}\left[B_{a, b}(G)\right]=\mathbb{P}\left[a<p\left(G ; \boldsymbol{L}_{\mathbf{2 n + 1}}\right)<b\right] ;
\end{aligned}
$$

where $\boldsymbol{L}_{\mathbf{2 n + 1}}$ is the random $A$-flag $\left(R_{2 n+1}, \boldsymbol{\theta}\right)$.

Note that since $\boldsymbol{\theta}$ is an embedding of $A$ in $R_{2 n+1}$, we must have

$$
\boldsymbol{\theta}(2)=(\boldsymbol{\theta}(1)+\boldsymbol{i}) \bmod (2 n+1),
$$

for some (random) $\boldsymbol{i} \in[n]$. Note also that from the symmetry of $R_{2 n+1}$, the variable $\boldsymbol{i}$ has uniform distribution in $[n]$.

Let $j \in[2 n]$ and $\boldsymbol{J}=\{\boldsymbol{\theta}(1), \boldsymbol{\theta}(2),(\boldsymbol{\theta}(1)+j) \bmod (2 n+1)\}$. Note that we have the following (see Figure 3.1).

- If $j<\boldsymbol{i}$, then $\boldsymbol{J}$ induces an occurrence of $\operatorname{Tr}_{3}^{A}$ in $\boldsymbol{L}_{\mathbf{2 n + 1}}$;

- If $\boldsymbol{i}<j \leqslant n$, then $\boldsymbol{J}$ induces an occurrence of $O^{A}$ in $\boldsymbol{L}_{\mathbf{2 n + 1}}$;

- If $n<j \leqslant \boldsymbol{i}+n$, then $\boldsymbol{J}$ induces an occurrence of $\vec{C}_{3}^{A}$ in $\boldsymbol{L}_{\mathbf{2 n + 1}}$;

- If $\boldsymbol{i}+n<j$, then $\boldsymbol{J}$ induces an occurrence of $I^{A}$ in $\boldsymbol{L}_{\mathbf{2 n + 1}}$. 


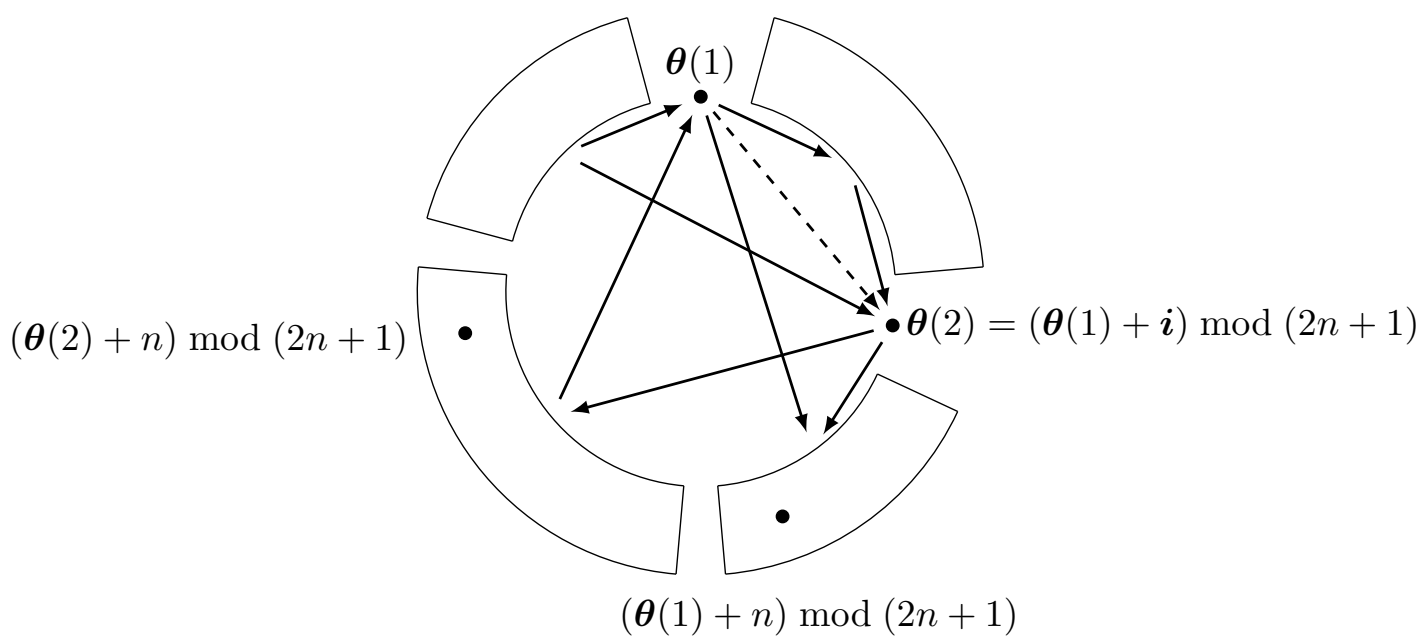

Figure 3.1: Neighbourhoods of $\boldsymbol{\theta}(1)$ and $\boldsymbol{\theta}(2)$.

This implies that

$$
\begin{aligned}
p\left(\operatorname{Tr}_{3}^{A} ; \boldsymbol{L}_{\mathbf{2 n + 1}}\right) & =\frac{\boldsymbol{i}-1}{2 n-1} ; & p\left(O^{A} ; \boldsymbol{L}_{\mathbf{2 n + 1}}\right) & =\frac{n-\boldsymbol{i}}{2 n-1} \\
p\left(\vec{C}_{3}^{A} ; \boldsymbol{L}_{\mathbf{2 n + 1}}\right) & =\frac{\boldsymbol{i}}{2 n-1} ; & p\left(I^{A} ; \boldsymbol{L}_{\mathbf{2 n + 1}}\right) & =\frac{n-\boldsymbol{i}}{2 n-1} .
\end{aligned}
$$

Hence, since $\boldsymbol{i}$ has uniform distribution over [n], we get

- $p\left(\operatorname{Tr}_{3}^{A} ; \boldsymbol{L}_{\mathbf{2 n + 1}}\right), p\left(O^{A} ; \boldsymbol{L}_{\mathbf{2 n + 1}}\right)$ and $p\left(I^{A} ; \boldsymbol{L}_{\mathbf{2 n + 1}}\right)$ have uniform distribution over the set $\{t /(2 n-1): t \in\{0,1, \ldots, n-1\}\}$;

- $p\left(\vec{C}_{3}^{A} ; \boldsymbol{L}_{\mathbf{2 n + 1}}\right)$ has uniform distribution over $\{t /(2 n-1): t \in[n]\}$;

- $p\left(O^{A}+I^{A} ; \boldsymbol{L}_{\mathbf{2 n + 1}}\right)$ has uniform distribution over $\{2 t /(2 n-1): t \in\{0,1, \ldots, n-1\}\}$;

- $p\left(\operatorname{Tr}_{3}^{A}+\vec{C}_{3}^{A} ; \boldsymbol{L}_{\mathbf{2 n + 1}}\right)$ has uniform distribution over $\left.\{(2 t-1) /(2 n-1): t \in[n]\}\right\}$.

Letting $n \rightarrow \infty$, it follows that

$$
\lim _{n \rightarrow \infty} \mathbb{P}_{R_{2 n+1}}^{A}\left[B_{a, b}(F)\right]=2(b-a),
$$

for every $0 \leqslant a \leqslant b \leqslant 1 / 2$;

$$
\lim _{n \rightarrow \infty} \mathbb{P}_{R_{2 n+1}}^{A}\left[B_{a, b}(F)\right]=1-2 a
$$

for every $0 \leqslant a \leqslant 1 / 2 \leqslant b \leqslant 1$; and

$$
\lim _{n \rightarrow \infty} \mathbb{P}_{R_{2 n+1}}^{A}\left[B_{a, b}(G)\right]=b-a,
$$

for every $0 \leqslant a \leqslant b \leqslant 1$ as desired.

Lemma 3.3.6 (C. [Cor15]). If $F \in \mathcal{F}_{3}^{A}$ is an $A$-flag of size 3 and $G \in \mathcal{A}^{A}$ is either $O^{A}+I^{A}$ or $\operatorname{Tr}_{3}^{A}+\vec{C}_{3}^{A}$, then we have

$$
S_{5}(F, 1 / 2) \Longrightarrow S_{6}(F, 1 / 4) \Longrightarrow S_{1}
$$


and

$$
S_{5}(G, 1) \Longrightarrow S_{6}(G, 1 / 2) \Longrightarrow S_{1} \text {. }
$$

Proof. First note that if $\phi \in \operatorname{Hom}^{+}\left(\mathcal{A}^{0}, \mathbb{R}\right)$, then

$$
\begin{array}{r}
\mathbb{E}\left[\phi^{A}\left(O^{A}\right)\right]=\mathbb{E}\left[\phi^{A}\left(I^{A}\right)\right]=\mathbb{E}\left[\phi^{A}\left(\operatorname{Tr}_{3}^{A}\right)\right]=\frac{\phi\left(\operatorname{Tr}_{3}\right)}{3} ; \\
\mathbb{E}\left[\phi^{A}\left(\vec{C}_{3}^{A}\right)\right]=\phi\left(\vec{C}_{3}\right)
\end{array}
$$

This implies that the conditions over the expected values in $S_{6}(F, 1 / 4)$ and $S_{6}(G, 1 / 2)$ are equivalent to $\phi$ being balanced (by $Q_{1}$ ).

Furthermore, since the expected values of random variables with distributions $U(0,1 / 2)$ and $U(0,1)$ are $1 / 4$ and $1 / 2$ respectively, we know that a homomorphism $\phi$ satisfying either $S_{5}(F, 1 / 2)$ or $S_{5}(G, 1)$ must be balanced.

Note now that for every balanced homomorphism $\phi$, we have

$$
\begin{aligned}
\mathbb{E}\left[\phi^{\boldsymbol{A}}\left(O^{A}\right)^{2}\right]=\mathbb{E}\left[\boldsymbol{\phi}^{\boldsymbol{A}}\left(I^{A}\right)^{2}\right]=\mathbb{E}\left[\boldsymbol{\phi}^{\boldsymbol{A}}\left(\operatorname{Tr}_{3}^{A}\right)^{2}\right] & =\frac{\phi\left(\operatorname{Tr}_{4}\right)}{6}=\frac{\phi\left(R_{4}\right)}{6} ; \\
\mathbb{E}\left[\boldsymbol{\phi}^{\boldsymbol{A}}\left(\vec{C}_{3}^{A}\right)^{2}\right] & =\frac{\phi\left(R_{4}\right)}{6} ; \\
\mathbb{E}\left[\boldsymbol{\phi}^{\boldsymbol{A}}\left(O^{A}+I^{A}\right)^{2}\right] & =\frac{\phi\left(\operatorname{Tr}_{4}\right)}{2}+\frac{\phi\left(R_{4}\right)}{6}=\frac{2 \phi\left(R_{4}\right)}{3} ; \\
\mathbb{E}\left[\phi^{\boldsymbol{A}}\left(\operatorname{Tr}_{3}^{A}+\vec{C}_{3}^{A}\right)^{2}\right] & =\frac{\phi\left(\operatorname{Tr}_{4}\right)}{6}+\frac{\phi\left(R_{4}\right)}{2}=\frac{2 \phi\left(R_{4}\right)}{3}
\end{aligned}
$$

From Corollary 3.3.3, we get that

$$
\mathbb{E}\left[\phi^{\boldsymbol{A}}(F)^{2}\right] \leqslant \frac{1}{12} \quad \mathbb{E}\left[\boldsymbol{\phi}^{\boldsymbol{A}}(G)^{2}\right] \leqslant \frac{1}{3},
$$

for every balanced homomorphism $\phi$, with equality if and only if $\phi=\phi_{\mathrm{R}}$.

Since the second moments of random variables with distributions $U(0,1 / 2)$ and $U(0,1)$ are $1 / 12$ and $1 / 3$ respectively, the result follows.

This concludes the proof of Theorem 3.2.2 (modulo Lemma 3.2.1). We will now present a proof of Lemma 3.2.1 that follows from a careful analysis of the proof of Lemma 3.3.1.

Proof of Lemma 3.2.1. From compactness of $[0,1]^{\mathcal{F}^{0}}$, we know that $\left(R_{2 n+1}\right)_{n \in \mathbb{N}}$ must have a convergent subsequence, so for every infinite set $I \subset \mathbb{N}$ of indexes such that the subsequence $\left(R_{2 i+1}\right)_{i \in I}$ converges, let $\phi_{I} \in \operatorname{Hom}^{+}\left(\mathcal{A}^{0}, \mathbb{R}\right)$ be its limit. For convenience, let $\mathcal{C}$ be the set of all $I \subset \mathbb{N}$ such that $\left(R_{2 i+1}\right)_{i \in I}$ converges.

Now we repeat the proof of Lemma 3.3.1 using an arbitrary $I \in \mathcal{C}$.

For the forward implication $S_{1} \Longrightarrow S_{2}$, since $R_{2 n+1}$ is a locally transitive balanced tournament, we have that $\phi_{I}$ is balanced and locally transitive for every $I \in \mathcal{C}$.

The proof of implication $S_{2} \Longrightarrow S_{1}$ proceeds a little bit differently: we pick the sequence $\left(T_{n}\right)_{n \in \mathbb{N}}$ of locally transitive tournaments converging to $\phi$ to be such that

$$
\left\{\left|T_{n}\right|: n \in \mathbb{N}\right\} \subset\{2 i+1: i \in I\} .
$$

This can be done by picking a sequence of sizes $\left(s_{n}\right)_{n \in \mathbb{N}}$ such that $s_{n}=\Omega\left(n^{2}\right)$ and $s_{n} \in$ $\{2 i+1: i \in I\}$ for every $n \in \mathbb{N}$ (see Corollary 1.2.19). 
Again, since $\phi$ is balanced, we know that we can obtain $R_{\left|T_{n}\right|}$ from $T_{n}$ by flipping $o\left(\left|T_{n}\right|^{2}\right)$ arcs of $T_{n}$ and since this flipping operation does not change the limit homomorphism, we have that the sequence $\left(T_{n}\right)_{n \in \mathbb{N}}$ converges to the same limit as a subsequence of $\left(R_{2 i+1}\right)_{i \in I}$, hence $\phi=\phi_{I}$.

But this means that, if $I, J \in \mathcal{C}$, then, we have

$$
S_{2}\left(\phi_{J}\right) \Longrightarrow \phi_{J}=\phi_{I},
$$

hence every convergent subsequence of $\left(R_{2 n+1}\right)_{n \in \mathbb{N}}$ converges to the same homomorphism, therefore it must be a convergent sequence by compactness of $[0,1]^{\mathcal{F}^{0}}$. 


\section{Chapter 4}

\section{Final remarks}

Let us finish this text by briefly commenting the results it contains and stating some related conjectures.

First, one may argue that, although Theorem 2.2.2 has an elegant proof, it is a somewhat expected result: since transitive tournaments have a nice organized structure, the natural way of minimizing its density is to consider the chaotic quasi-random tournament sequence. However, if we transpose this thought to other theories, we see that quasi-randomness is not the minimizer of the analogous objects.

For instance, the analogous problem in the Theory of Graphs is the problem of 2-common graphs, whose definition is below.

Definition 4.1. Let $\phi_{\mathrm{Graph}, \mathrm{qr}} \in \operatorname{Hom}^{+}\left(\mathcal{A}^{0}\left[T_{\mathrm{Graph}}\right], \mathbb{R}\right)$ denote the homomorphism corresponding to the almost sure limit of the sequence of Erdös-Rényi random graphs ${ }^{1}\left(\boldsymbol{G}_{\boldsymbol{n}, \mathbf{1} / \mathbf{2}}\right)_{n \in \mathbb{N}}$ of parameter $1 / 2$, that is, we have

$$
\phi_{\text {Graph,qr }}(G)=\frac{\ell !}{|\operatorname{Aut}(G)| 2^{\left(\begin{array}{c}
\ell \\
2
\end{array}\right)}},
$$

for every graph $G \in \mathcal{M}_{\ell}\left[T_{\text {Graph }}\right]$ of size $\ell$.

A graph $G$ is 2 -common ${ }^{2}$ if the density of $G+\bar{G}$ is minimized by $\phi_{\mathrm{Graph}, \mathrm{qr}}$, that is, if

$$
\min \left\{\psi(G+\bar{G}): \psi \in \operatorname{Hom}^{+}\left(\mathcal{A}^{0}\left[T_{\mathrm{Graph}}\right], \mathbb{R}\right)\right\}=\phi_{\text {Graph,qr }}(G+\bar{G}) .
$$

The analogous problem for $T_{\text {Graph }}$ is then the following conjecture by Erdős.

Conjecture 4.2 (Erdős [Erd62]). For every $k \in \mathbb{N}$, the complete graph $K_{k}$ of size $k$ is 2common.

Goodman [Goo59] proved this conjecture to be true for $k=3$ (note that the cases $k=$ 0,1,2 are trivial). However, Thomason [Tho89] proved this conjecture to be false for $k \geqslant 4$.

Another example of a theory where this intuition on quasi-randomness fails is that of $T_{\text {Perm }}$. The analogous conjecture is the following.

Conjecture 4.3. Let $\phi_{\text {Perm,qr }} \in \operatorname{Hom}^{+}\left(\mathcal{A}^{0}\left[T_{\text {Perm }}\right], \mathbb{R}\right)$ denote the homomorphism corresponding to the almost sure limit of the sequence $\left(\boldsymbol{\tau}_{\boldsymbol{n}}\right)_{n \in \mathbb{N}}$, where $\boldsymbol{\tau}_{\boldsymbol{n}}$ is picked uniformly at random

\footnotetext{
${ }^{1} \boldsymbol{G}_{\boldsymbol{n}, \boldsymbol{p}}$ is the random graph on $n$ vertices where each edge is present with probability $p$ independently of other edges.

${ }^{2}$ As suggested by the 2 in the terminology, there is actually a more general definition: A graph $G$ is $k$-common if the monochromatic density of $G$ in a $k$-edge-colouring of $K_{n}$ is asymptotically minimized if the colouring is random.
} 
in $\mathfrak{S}_{n}$ independently for each $n \in \mathbb{N}$, that is, we have

$$
\phi_{\mathrm{Perm}, \mathrm{qr}}(\tau)=\frac{1}{\ell !}
$$

for every permutation $\tau \in \mathfrak{S}_{\ell}$ of size $\ell$.

If $\operatorname{Id}_{k}$ and $\operatorname{Rev}_{k}$ denote the identity permutation of size $k$ and the decreasing permutation (also called reverse permutation) of size $k$ respectively, then for every $k \in \mathbb{N}$, the

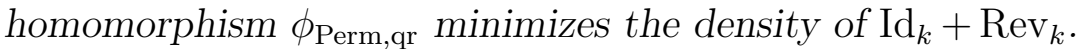

Unfortunately, this conjecture is also false as can be seen from the following simple example.

Lemma 4.4. Let $k \geqslant 2$ be an integer and for every $n \in \mathbb{N}$, let $\sigma_{n}$ be the permutation

$$
\begin{aligned}
& (n(n-1)(n-2) \cdots 1 \\
& (2 n)(2 n-1) \cdots(n+1) \\
& \quad \cdots \\
& ((k-1) n)((k-1) n-1) \cdots((k-2) n+1)) .
\end{aligned}
$$

Under these circumstances, the sequence $\left(\sigma_{n}\right)_{n \in \mathbb{N}}$ converges to a homomorphism $\phi_{k} \in$ $\operatorname{Hom}^{+}\left(\mathcal{A}^{0}\left[T_{\text {Perm }}\right], \mathbb{R}\right)$ and

$$
\phi_{k}\left(\operatorname{Id}_{k}+\operatorname{Rev}_{k}\right)=\frac{1}{(k-1)^{k-1}} .
$$

Note that for $k \geqslant 3$, we have $1 /(k-1)^{k-1}<2 / k$ !, which implies that Conjecture 4.3 is only true for the trivial cases $k=0,1,2$.

In fact, Myers [Mye03] conjectured that $\phi_{k}$ is the correct minimizing homomorphism. We state this conjecture below.

Conjecture 4.5 (Myers [Mye03]). In the Theory of Permutations, if $k \geqslant 2$ is an integer, then

$$
\min \left\{\phi\left(\operatorname{Id}_{k}+\operatorname{Rev}_{k}\right): \phi \in \operatorname{Hom}^{+}\left(\mathcal{A}^{0}\left[T_{\text {Perm }}\right], \mathbb{R}\right)\right\}=\frac{1}{(k-1)^{k-1}}
$$

Myers [Mye03] proved that this conjecture holds for the case $k=3$ and the case $k=4$ was proved in $\left[\mathrm{BHL}^{+} 15\right]$ using the semidefinite method of flag algebras (see Section B.1).

Returning to the Theory of Tournaments, as we mentioned before, it is not that surprising that the minimizer of a fixed structure is quasi-random, so a natural question is the following.

Question 4.6. Does there exist a tournament $T$ whose density is maximized only by the quasi-random homomorphism $\phi_{\mathrm{qr}}$ ? And if so, then what do tournaments $T$ satisfying this property look like?

To make the second part of the question above more precise, let us state another question related to the maximization problem.

Question 4.7. In a universal theory $T$, let $\phi \in \operatorname{Hom}^{+}\left(\mathcal{A}^{0}, \mathbb{R}\right)$ be a homomorphism and let $\left(M_{n}\right)_{n \in \mathbb{N}}$ be an increasing sequence of models of $T$ such that for every $n \in \mathbb{N}$, the homomorphism $\phi$ is the only maximizer of the density of $M_{n}$, that is, we have

$$
\forall \psi \in \operatorname{Hom}^{+}\left(\mathcal{A}^{0}, \mathbb{R}\right), \psi\left(M_{n}\right) \leqslant \phi\left(M_{n}\right),
$$


with equality if and only if $\phi=\psi$.

Under these circumstances, is it true that $\left(M_{n}\right)_{n \in \mathbb{N}}$ converges to $\phi$ ? And if not, can we say at least that $\left(M_{n}\right)_{n \in \mathbb{N}}$ bears some structural similarity with $\phi$ ?

A trivial example of this convergence is the sequence of transitive tournaments $\left(\operatorname{Tr}_{n}\right)_{n \in \mathbb{N}}$, but the next two theorems show some non-trivial examples concerning graph blow-ups.

Definition 4.8. If $G$ is a graph with $V(G)=[n]$ and $\vec{k}=\left(k_{1}, k_{2}, \ldots, k_{n}\right) \in \mathbb{N}^{n}$ is a vector of $n$ positive integers, then the $\vec{k}$-blow-up of $G$, denoted by $G^{(\vec{k})}$ is the graph obtained by replacing each vertex $i \in[n]$ of $G$ with $k_{i}$ copies of it and replacing each edge by a complete bipartite graph. Formally, we have

$$
\begin{aligned}
& V\left(G^{(\vec{k})}\right)=\left\{(i, j): i \in[n] \wedge j \in\left[k_{i}\right]\right\} \\
& E\left(G^{(\vec{k})}\right)=\left\{\left(i_{1}, j_{1}\right)\left(i_{2}, j_{2}\right): i_{1} i_{2} \in E(G)\right\}
\end{aligned}
$$

Furthermore, if $k>0$, we define $G^{(k)}=G^{((k, k, \ldots, k))}$.

Theorem 4.9 (Bollobás-Egawa-Harris-Jin[BEHJ95]). If $r>0$, then there exists $h_{0} \in \mathbb{N}$ such that if $h \geqslant h_{0}$, then the density of $K_{r}^{(h)}$ is maximized by the limit of the sequence $\left(K_{r}^{(n)}\right)_{n \in \mathbb{N}}$.

Theorem 4.10 (Hatami-Hirst-Norine [HHN14]). If $G$ is a graph, then there exists $h_{0} \in \mathbb{N}$ such that if $h \geqslant h_{0}$, then there exists a sequence of vectors $(\vec{k}(n))_{n \in \mathbb{N}}$ such that the density of $G^{(h)}$ is maximized by the limit of the sequence $\left(G^{(\vec{k}(n))}\right)_{n \in \mathbb{N}}$.

With Question 4.7 in mind and noting that tournament $R_{4}$ bears some structural similarity with the carousel tournaments $R_{2 n+1}$, we make the following two conjectures.

Conjecture 4.11 ([Cor15, Conjecture 5.4]). If $n \in \mathbb{N}$, then the carousel homomorphism $\phi_{\mathrm{R}}$ maximizes the density of the carousel tournament $R_{2 n+1}$.

Conjecture 4.12 ([Cor15, Conjecture 5.5]). If $n \geqslant 2$, then the carousel homomorphism $\phi_{\mathrm{R}}$ is the only maximizer of the density of the carousel tournament $R_{2 n+1}$.

Finally, let us finish this chapter with a more concrete conjecture in the Theory of Tournaments.

As we briefly mentioned in the introduction, Corollary 2.2.5 closes the problem of minimizing the density of a fixed tournament $T$. Furthermore, Corollary 3.3.3 closes the problem of maximizing the density of $R_{4}$. This leaves only one case of size 4 still open since maximizing the density of $W_{4}$ is analogous to maximizing the density of $L_{4}$ by flipping all arcs.

For the particular problem of $W_{4}$, consider the following construction (see Figure 4.1). Let $N$ be an arbitrarily large integer and $t \in(0,1)$. Define recursively the sequence $A_{0}, A_{1}, \ldots$ by taking $A_{0}=[N]$ and by letting $A_{i}$ be a subset of $A_{i-1}$ with size $t\left|A_{i}\right|$ (rounded to the nearest integer) for every $i>0$. Define the random tournament $\boldsymbol{S}_{N, t}$ through the following procedure: let $V\left(\boldsymbol{S}_{\boldsymbol{N}, \boldsymbol{t}}\right)=[N]=A_{0}$; for every $i>0$, every $v \in A_{i}$ and every $w \in A_{i-1} \backslash A_{i}$, let $(v, w) \in A\left(\boldsymbol{S}_{\boldsymbol{N}, \boldsymbol{t}}\right)$ and pick all the remaining arc orientations independently at random with probability $1 / 2$. That is, for every $i>0$, if $k=\left|A_{i-1} \backslash A_{i}\right|$, then the set $A_{i-1} \backslash A_{i}$ spans $\boldsymbol{R}_{\boldsymbol{k}, \mathbf{1} / \mathbf{2}}$.

It is (somewhat) easy to see that $\left(\boldsymbol{S}_{\boldsymbol{N}, \boldsymbol{t}}\right)_{N \in \mathbb{N}}$ converges almost surely to a limit homomorphism $\phi_{t}$ such that

$$
\phi_{t}\left(W_{4}\right)=(1-t)^{3}\left(t+\frac{1-t}{8}\right) /\left(1-t^{4}\right) .
$$




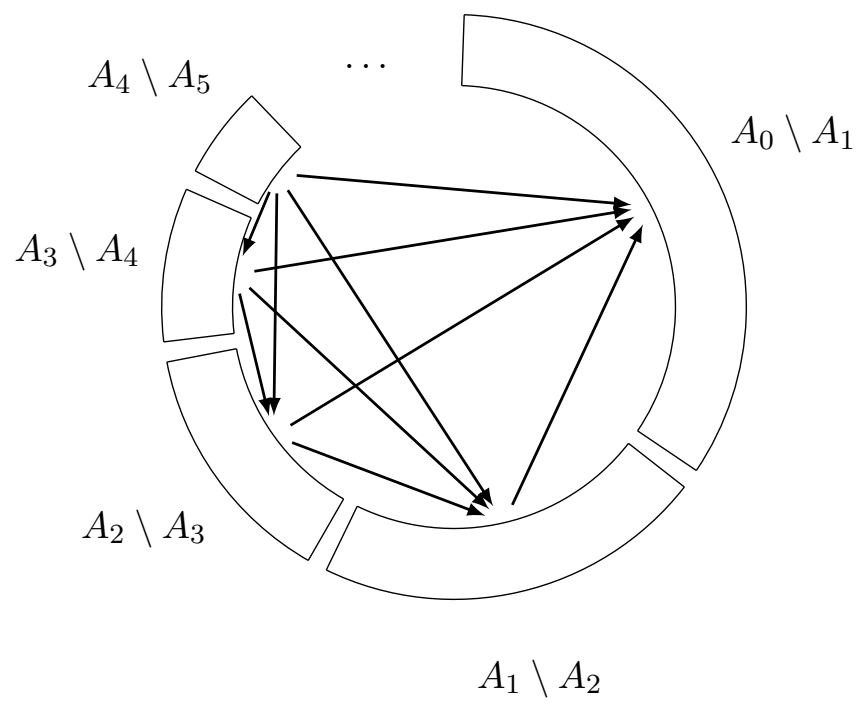

Figure 4.1: Typical structure of the random tournament $\boldsymbol{S}_{\boldsymbol{N}, \boldsymbol{t}}$. The arcs in the picture represent arcs between vertices in distinct parts $A_{i-1} \backslash A_{i}$. The arcs completely contained any part $A_{i-1} \backslash A_{i}$ have their orientation picked independently at random with probability $1 / 2$ for each orientation. This figure uses $t=0.65$, which makes it easier to see the structure of the construction but is far from the value of $t$ that maximizes $\phi_{t}\left(W_{4}\right)$.

Certainly, every value of $\phi_{t}\left(W_{4}\right)$ for $t \in(0,1)$ is a lower bound for the maximization problem for $W_{4}$. The maximum of $\phi_{t}\left(W_{4}\right)$ (which can be computed with standard calculus arguments) is

$$
\max \left\{\phi_{t}\left(W_{4}\right): t \in(0,1)\right\}=1+\frac{3^{5 / 3}-3^{7 / 3}}{8} \approx 0.157501
$$

attained when $t$ is equal to

$$
\frac{2 \cdot 3^{2 / 3}-3^{1 / 3}-2}{5} \approx 0.143584
$$

We conjecture that this is actually the maximum value of $\phi\left(W_{4}\right)$ for $\phi \in \operatorname{Hom}^{+}\left(\mathcal{A}^{0}, \mathbb{R}\right)$.

Conjecture 4.13. In the Theory of Tournaments, we have

$$
\max \left\{\phi\left(W_{4}\right): \phi \in \operatorname{Hom}^{+}\left(\mathcal{A}^{0}, \mathbb{R}\right)\right\}=1+\frac{3^{5 / 3}-3^{7 / 3}}{8} .
$$

The intuition of the recursive construction of $\boldsymbol{S}_{N, t}$ is that at every step we have one part $A_{i-1} \backslash A_{i}$ that maximizes the density of $\vec{C}_{3}$ (hence almost balanced in the sense that this part converges to a balanced homomorphism) and another part $A_{i}$ whose vertices all beat the first part. This maximizes the occurrences of $W_{4}$ with exactly one vertex in the latter part, and since only one vertex is being selected in it, we might as well repeat this structure recursively in $A_{i}$.

In this particular construction, we chose the (almost) balanced part to be quasi-random. However, one might wonder if this is the best we can do in the class of balanced homomorphisms to maximize the density of $W_{4}$, but the following couple of lemmas show that this is indeed the case. 
Lemma 4.14. In the Theory of Tournaments, if $\phi \in \operatorname{Hom}^{+}\left(\mathcal{A}^{0}, \mathbb{R}\right)$ is balanced, then $\phi\left(W_{4}\right)=$ $\phi\left(L_{4}\right)$.

Proof. Since $\phi$ is balanced, we have $\phi^{\mathbf{1}}(\alpha)=\phi^{\mathbf{1}}(\beta)$ a.s. In particular, this means that

$$
\frac{\phi\left(\operatorname{Tr}_{4}+W_{4}\right)}{4}=\mathbb{E}\left[\phi^{\mathbf{1}}(\alpha)^{3}\right]=\mathbb{E}\left[\phi^{\mathbf{1}}(\beta)^{3}\right]=\frac{\phi\left(\operatorname{Tr}_{4}+L_{4}\right)}{4},
$$

hence $\phi\left(W_{4}\right)=\phi\left(L_{4}\right)$.

Lemma 4.15. In the Theory of Tournaments, if $\phi \in \operatorname{Hom}^{+}\left(\mathcal{A}^{0}, \mathbb{R}\right)$ is balanced, then $\phi\left(W_{4}\right) \leqslant$ $1 / 8$ with equality if and only if $\phi$ is the quasi-random homomorphism $\phi_{\mathrm{qr}}$.

Proof. Quasi-random property $P_{2}$ says that if $\psi \in \operatorname{Hom}^{+}\left(\mathcal{A}^{0}, \mathbb{R}\right)$, then $\psi\left(\operatorname{Tr}_{4}+R_{4}\right) \geqslant 3 / 4$ with equality if and only if $\psi=\phi_{\mathrm{qr}}$, hence $\psi\left(W_{4}+L_{4}\right) \leqslant 1 / 4$ with equality if and only if $\psi=\phi_{\mathrm{qr}}$.

On the other hand, since $\phi$ is balanced, Lemma 4.14 implies that $\phi\left(W_{4}\right)=\left(\phi\left(W_{4}+\right.\right.$ $\left.\left.L_{4}\right)\right) / 2 \leqslant 1 / 8$.

Since $\phi_{\mathrm{qr}}$ is also balanced, the result follows. 


\section{Appendix A}

\section{First-order logic}

Definition A.1. An open formula $\phi$ over a finite first-order language is a formula that contains no quantifiers.

A closed formula $\phi$ over a finite first-order language is a formula that contains no free variables.

Definition A.2. Let $M$ be a model of a theory $T$ over a finite first-order language $L \cup\{=\}$ with equality $=$ and $W \subset V(M)$ be such that

i. For every constant symbol $c$ of $L$, we have $M \models c \in W$;

ii. For every $k$-ary function symbol $f$ of $L$, we have $M \models f\left(W^{k}\right) \subset W$.

Under these circumstances, the model restriction of $M$ to $W$ is denoted by $\left.M\right|_{W}$ and is defined by

- $V\left(\left.M\right|_{W}\right)=W$;

- $\left.M\right|_{W}=p\left(v_{1}, v_{2}, \ldots, v_{k}\right)$ if and only if $M \models p\left(v_{1}, v_{2}, \ldots, v_{k}\right)$, for every $k$-ary predicate symbol $p$ of $L$ and all vertices $v_{1}, v_{2}, \ldots, v_{k} \in W$;

- $\left.M\right|_{W}=c=v$ if and only if $M \models c=v$, for every constant symbol $c$ of $L$ and every vertex $v \in W$;

- $\left.M\right|_{W} \models f\left(v_{1}, v_{2}, \ldots, v_{k}\right)=w$ if and only if $M \models f\left(v_{1}, v_{2}, \ldots, v_{k}\right)=w$ for every $k$-ary function symbol $f$ of $L$ and all vertices $v_{1}, v_{2}, \ldots, v_{k}, w \in W$;

Note that although conditions (i) and (ii) are enough to make $\left.M\right|_{W}$ a model over $L$, it is not necessarily a model of $T$.

Definition A.3. A theory $T$ over a finite first-order language $L$ is a universal theory if all of its axioms are of the form

$$
\forall x_{1} \forall x_{2} \cdots \forall x_{k}, \phi\left(x_{1}, x_{2}, \ldots, x_{k}\right)
$$

where $\phi$ is an open formula.

Proposition A.4. Under the conditions of Definition A.2, if $T$ is also a universal theory, then $\left.M\right|_{W}$ is a model of $T$ (called model induced by $W$ in $M$ ).

Proof. Since $T$ is universal, every axiom of $T$ is of the form

$$
\forall x_{1} \forall x_{2} \cdots \forall x_{k}, \phi
$$


for some $k \in \mathbb{N}$ and some open formula $\phi$. By restricting the universal quantification to $W$, we get in particular that $\left.M\right|_{W} \models T$.

Definition A.5. Let $M_{1}$ and $M_{2}$ be models of a universal theory $T$ over a language $L$ with equality and no function symbols. A model embedding of $M_{1}$ in $M_{2}$ is an injective function $f: V\left(M_{1}\right) \rightarrow V\left(M_{2}\right)$ such that

- For every $k$-ary predicate symbol $p \in L$ and for all vertices $v_{1}, v_{2}, \ldots, v_{k} \in V\left(M_{1}\right)$, we have

$$
M_{1}=p\left(v_{1}, v_{2}, \ldots, v_{k}\right) \Longleftrightarrow M_{2}=p\left(f\left(v_{1}\right), f\left(v_{2}\right), \ldots, f\left(v_{k}\right)\right)
$$

- For every constant symbol $c \in L$ and every vertex $v \in V\left(M_{1}\right)$, we have

$$
M_{1} \models c=v \Longleftrightarrow M_{2} \models c=f(v) .
$$

An isomorphism between $M_{1}$ and $M_{2}$ is a bijective model embedding of $M_{1}$ in $M_{2}$. them.

Two models of a universal theory are isomorphic if there exists an isomorphism between

An automorphism of a model $M$ is an isomorphism between $M$ and $M$.

Definition A.6. If $M$ is a model with $V(M)=[k]$ (i.e., a type in flag algebra) over the first-order language $L$, then the open diagram of $M$ over the variables $x_{1}, x_{2}, \ldots, x_{k}$ is the conjunction of all literals of the form

$$
q\left(x_{i_{1}}, x_{i_{2}}, \ldots, x_{i_{m}}\right)
$$

where $q$ is either $p$ or $\neg p$ for a $m$-ary predicate $p$ of $L$ and $i_{1}, i_{2}, \ldots, i_{m}$ are such that

$$
M \models q\left(i_{1}, i_{2}, \ldots, i_{m}\right) .
$$

By abuse of terminology, we say that a formula $\phi$ is an open diagram of a model $M$ over any finite set of vertices if it is the open diagram of a model $N$ over $[|V(M)|]$ that is isomorphic to $M$.

Example A.7. In $T_{\text {Graph }}$, an open diagram of the triangle $K_{3}$ over $x, y, z$ is

$$
\begin{gathered}
e(x, y) \wedge e(y, z) \wedge e(z, x) \wedge e(y, x) \wedge e(z, y) \wedge e(x, z) \wedge \neg e(x, x) \wedge \neg e(y, y) \wedge \neg e(z, z) \\
\wedge x=x \wedge y=y \wedge z=z \wedge \neg(x=y) \wedge \neg(y=z) \wedge \neg(z=x) \neg(y=x) \wedge \neg(z=y) \wedge \neg(x=z) .
\end{gathered}
$$

Definition A.8. Let $L_{1}$ and $L_{2}$ be two languages with equality and no function symbols (but they may contain constant symbols).

A translation of $L_{1}$ to $L_{2}$ is a function $I$ defined on all symbols of $L_{1}$ that are not logical connectives such that

- I fixes variables;

- I takes every $k$-ary predicate symbol $p\left(x_{1}, x_{2}, \ldots, x_{k}\right)$ of $L_{1}$ to a well-formed formula $I(p)\left(x_{1}, x_{2}, \ldots, x_{k}\right)$ of $L_{2}$ such that only the variables $x_{1}, x_{2}, \ldots, x_{k}$ may be free in $I(p)\left(x_{1}, x_{2}, \ldots, x_{k}\right)$;

- I takes every constant symbol $c \in L_{1}$ to a constant symbol $I(c)$ of $L_{2}$. 
We extend $I$ to the well-formed formulas of $L_{1}$ by declaring that it commutes with logical connectives.

Let $T_{1}$ and $T_{2}$ be universal theories in $L_{1}$ and $L_{2}$ respectively.

A pair $(U, I)$ is an open interpretation of $T_{1}$ in $T_{2}$, denoted by $(U, I): T_{1} \rightsquigarrow T_{2}$ if

- $U=U(x)$ is an open formula in $L_{2}$ such that only the variable $x$ may be free in $U(x)$;

- $I$ is a translation of $L_{1}$ to $L_{2}$ that takes every predicate symbol $p\left(x_{1}, x_{2}, \ldots, x_{k}\right)$ of $L_{1}$ to an open formula $I(p)\left(x_{1}, x_{2}, \ldots, x_{k}\right)$ of $L_{2}$;

- For every axiom $\forall x_{1} \forall x_{2} \cdots \forall x_{n} A\left(x_{1}, x_{2}, \ldots, x_{n}\right)$ of the theory $T_{1}$, we have

$$
T_{2} \vdash \forall x_{1} \forall x_{2} \cdots \forall x_{n},\left(U\left(x_{1}\right) \wedge U\left(x_{2}\right) \wedge \cdots \wedge U\left(x_{n}\right) \Longrightarrow I(A)\left(x_{1}, x_{2}, \ldots, x_{n}\right)\right) .
$$

A model of $T_{2}$ is a $U$-model if $M \models \forall x, U(x)$.

Finally, let $M$ be a $U$-model of $T_{2}$; we define the interpretation of the model $M$, denoted by $I(M)$ (also called model interpretation of $M$ ), as the model of $T_{1}$ such that

- The set of vertices of $I(M)$ is the same as the set of vertices of $M$, that is, we have $V(I(M))=V(M)$;

- For every $k$-ary predicate symbol $p$ of $L_{1}$ and all vertices $v_{1}, v_{2}, \ldots, v_{k} \in V(I(M))$, we have

$$
\left(I(M) \models p\left(v_{1}, v_{2}, \ldots, v_{k}\right)\right) \Longleftrightarrow\left(M \models I(p)\left(v_{1}, v_{2}, \ldots, v_{k}\right)\right) ;
$$

- For every constant symbol $c$ of $L_{1}$ and every vertex $v \in V(I(M))$, we have

$$
(I(M) \models v=c) \Longleftrightarrow(M \models v=I(c)) .
$$




\section{Appendix B}

\section{Other topics in flag algebra}

In this chapter, we will present some other important proof techniques in the theory of flag algebras. Namely, we will present the semidefinite method, which is a proof technique to obtain bounds in extremal problems as solutions of a semidefinite program; and the differential method, which provides a way of using analysis techniques to obtain properties of local minimizers (or maximizers).

In this chapter, we also suppose fixed a universal theory $T$ in a finite first-order language with equality and without constant or function symbols that has at least one infinite model.

\section{B.1 Semidefinite method}

We start with some useful notation in flag algebras.

Definition B.1. Let $\sigma$ be a non-degenerate type. The semantic cone relative to $\sigma$, denoted by $\mathcal{C}_{\mathrm{sem}}\left(\mathcal{F}^{\sigma}\right)$, is the set of all $\sigma$-flag algebra elements $f$ such that $\phi(f) \geqslant 0$ for every positive homomorphism $\phi \in \operatorname{Hom}^{+}\left(\mathcal{A}^{\sigma}, \mathbb{R}\right)$, that is, we have

$$
\mathcal{C}_{\text {sem }}\left(\mathcal{F}^{\sigma}\right)=\left\{f \in \mathcal{A}^{\sigma}: \forall \phi \in \operatorname{Hom}^{+}\left(\mathcal{A}^{\sigma}, \mathbb{R}\right), \phi(f) \geqslant 0\right\} .
$$

The ordinary cone relative to $\sigma$, denoted by $\mathcal{C}\left(\mathcal{F}^{\sigma}\right)$, is the set of all sums of elements of the form $f^{2} F$, where $f \in \mathcal{A}^{\sigma}$ and $F \in \mathcal{F}^{\sigma}$, that is, we have

$$
\mathcal{C}\left(\mathcal{F}^{\sigma}\right)=\left\{\sum_{i=1}^{m} f_{i}^{2} F_{i}: m \in \mathbb{N} \wedge f_{1}, f_{2}, \ldots, f_{m} \in \mathcal{A}^{\sigma} \wedge F_{1}, F_{2}, \ldots, F_{m} \in \mathcal{F}^{\sigma}\right\} .
$$

Finally we define the relation $\leqslant_{\sigma}$ on $\mathcal{A}^{\sigma}$ through

$$
f \leqslant_{\sigma} g \Longleftrightarrow g-f \in \mathcal{C}_{\mathrm{sem}}\left(\mathcal{F}^{\sigma}\right) .
$$

Clearly we have $\mathcal{F}^{\sigma} \subset \mathcal{C}\left(\mathcal{F}^{\sigma}\right) \subset \mathcal{C}_{\text {sem }}\left(\mathcal{F}^{\sigma}\right)$ (and clearly the latter two sets are cones). It is also easy to see that $\leqslant_{\sigma}$ is a preorder relation.

Deciding whether an element $f \in \mathcal{A}^{\sigma}$ is in the semantic cone $\mathcal{C}_{\text {sem }}\left(\mathcal{F}^{\sigma}\right)$ is a hard problem. In fact, it is proved in [HN11] that this problem is undecidable ${ }^{1}$.

However, elements of the ordinary cone are easy to obtain. The next theorems relates cones of different types and theories and will provide a very useful way of obtaining even more elements of the semantic cone.

\footnotetext{
${ }^{1}$ The original proof is in the theory of quantum graphs, but it is also translated to the theory of flag algebras.
} 
Theorem B.2 ([Raz07, Theorem 3.1a]). If $\left(\sigma_{2}, \eta\right)$ is a type extension of $\sigma_{1}$, then

$$
\llbracket \mathcal{C}_{\mathrm{sem}}\left(\mathcal{F}^{\sigma_{2}}\right) \rrbracket_{\sigma_{2}, \eta} \subset \mathcal{C}_{\mathrm{sem}}\left(\mathcal{F}^{\sigma_{1}}\right) .
$$

Theorem B.3 ([Raz07, Theorem 3.1b]). With the definitions and notation of Theorem 1.4.3, if $f \in \mathcal{C}_{\mathrm{sem}}\left(\mathcal{F}^{\sigma_{1}}\left[T_{1}\right]\right)$ and $\ell \in \mathbb{N}$ is such that

$$
u^{\ell} \pi^{(U, I)}(f) \in \mathcal{A}^{\sigma_{2}}\left[T_{2}\right]
$$

(that is, the denominator of this element is $\left.1_{\sigma_{2}}\right)$, then we have

$$
u^{\ell} \pi^{(U, I)}(f) \in \mathcal{C}_{\text {sem }}\left(\mathcal{F}^{\sigma_{2}}\left[T_{2}\right]\right) .
$$

Let us now present the semidefinite method. Consider the following extremal problem in flag algebras (which we state for type 0 for simplicity).

Problem B.4. Given $f \in \mathcal{A}^{0}$, compute

$$
\max \left\{\phi(f): \phi \in \operatorname{Hom}^{+}\left(\mathcal{A}^{0}, \mathbb{R}\right)\right\} .
$$

Furthermore, if $\phi \in \operatorname{Hom}^{+}\left(\mathcal{A}^{0}, \mathbb{R}\right)$, then what are its properties? Can we prove, for instance, that there is only one maximizing homomorphism?

Obtaining lower bounds for the value in this problem is easy: every $\psi \in \operatorname{Hom}^{+}\left(\mathcal{A}^{0}, \mathbb{R}\right)$ provides the lower bound $\psi(f)$. The semidefinite method provides a way of obtaining upper bounds for this problem. The idea is the following: we first choose $\ell$ large enough and write

$$
f=\sum_{F \in \mathcal{F}_{\ell}^{0}} p(f ; F) F \leqslant_{0}\left(\max _{F \in \mathcal{F}_{\ell}^{0}} p(f ; F)\right)\left(\sum_{F \in \mathcal{F}_{\ell}^{0}} F\right) \leqslant_{0}\left(\max _{F \in \mathcal{F}_{\ell}^{0}} p(f ; F)\right) 1_{0},
$$

hence we get $\psi(f) \leqslant \max _{F \in \mathcal{F}_{\ell}^{0}} p(f ; F)$ for every homomorphism $\psi \in \operatorname{Hom}^{+}\left(\mathcal{A}^{0}, \mathbb{R}\right)$. This bound is in general very bad, so to improve it we can suppose $g \in \mathcal{C}_{\text {sem }}\left(\mathcal{F}^{0}\right)$, then we have $f \leqslant_{0} f+g$, and we can repeat the procedure for $f+g$, possibly choosing a larger $\ell$ :

$$
\begin{aligned}
f & \leqslant 0 f+g=\sum_{F \in \mathcal{F}_{\ell}^{0}} p(f+g ; F) F \\
& \leqslant 0\left(\max _{F \in \mathcal{F}_{\ell}^{0}} p(f+g ; F)\right)\left(\sum_{F \in \mathcal{F}_{\ell}^{0}} F\right) \leqslant 0\left(\max _{F \in \mathcal{F}_{\ell}^{0}} p(f+g ; F)\right) 1_{0},
\end{aligned}
$$

and get $\psi(f) \leqslant \max _{F \in \mathcal{F}_{\ell}^{0}} p(f+g ; F)$ for every $\psi \in \operatorname{Hom}^{+}\left(\mathcal{A}^{0}, \mathbb{R}\right)$. Our hope in doing so is that $g$ has negative values $p(g ; F)$ for the flags such that $p(f ; F)$ is large.

With this idea in mind, we can restrict the element $g$ to the set $\llbracket \mathcal{C}\left(\mathcal{F}^{\sigma}\right) \rrbracket_{\sigma} \subset \mathcal{C}_{\text {sem }}\left(\mathcal{F}^{0}\right)$ and reduce the problem to finding a positive semidefinite matrix in the following way.

Fix a type $\sigma$ and $\tilde{\ell} \in \mathbb{N}$ with $|\sigma| \leqslant \widetilde{\ell}$ and $2 \widetilde{\ell}-|\sigma| \leqslant \ell$ (possibly making $\ell$ larger again) and let $\vec{F}=\left(F_{1}, F_{2}, \ldots, F_{m}\right)$ be an ordering of $\mathcal{F}_{\widetilde{\ell}}^{\sigma}$. If $A \succeq 0$ is a positive semidefinite matrix of order $m \times m$ with real values, then by the Spectral Theorem, we can write

$$
A=\sum_{i=1}^{r} v_{i} v_{i}^{\top}
$$


for vectors $v_{1}, v_{2}, \ldots, v_{r} \in \mathbb{R}^{m}$. Write $v_{i}=\left(w_{i 1}, w_{i 2}, \ldots, w_{i m}\right)$ and considering formal matrix products, we have

$$
v_{i}^{\top} \vec{F}=\sum_{j=1}^{m} w_{i j} F_{j}
$$

and

$$
\llbracket \vec{F}^{\top} A \vec{F} \|_{\sigma}=\llbracket \sum_{i=1}^{r} \vec{F}^{\top} v_{i} v_{i}^{\top} \vec{F} \rrbracket_{\sigma}=\llbracket \sum_{i=1}^{r}\left(\sum_{j=1}^{m} w_{i j} F_{j}\right)^{2} \rrbracket_{\sigma} \in \llbracket \mathcal{C}\left(\mathcal{F}^{\sigma}\right) \rrbracket_{\sigma} \subset \mathcal{C}_{\mathrm{sem}}\left(\mathcal{F}^{0}\right) .
$$

This allows us to write the following semidefinite program whose solutions are upper bounds for Problem B.4.

$\min y$

$$
\begin{array}{ll}
\text { s.t. } & y-\left(p(f ; G)+\sum_{F \in \mathcal{F}_{\ell}^{\sigma}} \sum_{i, j \in[m]} a_{i j} p\left(F_{i}, F_{j} ; F\right) p\left(\llbracket F \rrbracket_{\sigma} ; G\right)\right) \geqslant 0 \quad \forall G \in \mathcal{F}_{\ell}^{0} ; \\
& A=\left(a_{i j}\right)_{i, j=1}^{m} \succeq 0 .
\end{array}
$$

Before going into an example of this method, let us mention that we can use more than one type in the above formulation (that is, we can look for $g_{1}, g_{2}, \ldots, g_{t}$ with each $\left.g_{i} \in \llbracket \mathcal{C}\left(\mathcal{F}^{\sigma_{i}}\right) \rrbracket_{\sigma_{i}}\right)$ and we can also consider elements of the form

$$
\llbracket \widetilde{F} \cdot \vec{F}^{\top} A \vec{F} \|_{\sigma}
$$

in equation (B.1), where $\widetilde{F} \in \mathcal{F}^{\sigma}$ is a fixed $\sigma$-flag.

As an example of application of the semidefinite method, we will prove Proposition 2.3.2, which we restate as the proposition below. The second part of the proof will also illustrate how one can use the solution of the semidefinite program to infer properties of the extremal homomorphisms.

Proposition B.5 (Proposition 2.3.2). If $\phi \in \operatorname{Hom}^{+}\left(\mathcal{A}^{0}, \mathbb{R}\right)$, then

$$
\phi\left(\vec{C}_{3}\right) \leqslant \frac{1}{4}
$$

with equality if and only if $\boldsymbol{\phi}^{\mathbf{1}}(\alpha)=1 / 2$ a.s.

Proof. Note that

$$
\vec{C}_{3} \leqslant{ }_{0} \vec{C}_{3}+\llbracket \frac{3}{4}(\alpha-\beta)^{2} \rrbracket_{1}=\vec{C}_{3}+\frac{3}{4}\left(\frac{1}{3} \operatorname{Tr}_{3}-\vec{C}_{3}^{A}\right)=\frac{1}{4} \vec{C}_{3}+\frac{1}{4} \operatorname{Tr}_{3}=\frac{1_{0}}{4} .
$$

Furthermore, if $\phi$ is such that $\phi\left(\vec{C}_{3}\right)=1 / 4$, then we have

$$
\frac{1}{4}=\phi\left(\vec{C}_{3}\right) \leqslant \phi\left(\vec{C}_{3}+\llbracket \frac{3}{4}(\alpha-\beta)^{2} \rrbracket_{1}\right)=\frac{1}{4},
$$


hence $\phi\left(\llbracket(\alpha-\beta)^{2} \rrbracket_{1}\right)=0$, which implies that

$$
\mathbb{E}\left[\phi^{\mathbf{1}}(\alpha-\beta)^{2}\right]=\frac{\phi\left(\llbracket(\alpha-\beta)^{2} \rrbracket_{1}\right)}{\phi\left(\llbracket 1_{1} \rrbracket_{1}\right)}=0 .
$$

Therefore $\boldsymbol{\phi}^{\mathbf{1}}(\alpha)=\boldsymbol{\phi}^{\mathbf{1}}(\beta)=1 / 2$ a.s.

Finally, we will offer a semidefinite method proof of a part of Corollary 3.3.3 to illustrate another type of reasoning that can be made about solutions of the semidefinite program.

Proposition B.6 (Part of Corollary 3.3.3). For every $\phi \in \operatorname{Hom}^{+}\left(\mathcal{A}^{0}, \mathbb{R}\right)$, we have

$$
\phi\left(R_{4}\right) \leqslant \frac{1}{2}
$$

with equality if and only if $\phi$ is locally transitive.

Proof. Note that

$$
R_{4} \leqslant{ }_{0} R_{4}+\llbracket 6\left(\operatorname{Tr}_{3}^{A}-\vec{C}_{3}^{A}\right)^{2} \rrbracket_{A}=R_{4}+6\left(\frac{1}{12} \operatorname{Tr}_{4}-\frac{1}{12} R_{4}\right)=\frac{1}{2} \operatorname{Tr}_{4}+\frac{1}{2} R_{4} \leqslant 0 \frac{1_{0}}{2} .
$$

Furthermore, if $\phi$ is such that $\phi\left(R_{4}\right)=1 / 2$, then we have

$$
\frac{1}{2}=\phi\left(R_{4}\right) \leqslant \phi\left(R_{4}+\llbracket 6\left(\operatorname{Tr}_{3}^{A}-\vec{C}_{3}^{A}\right)^{2} \rrbracket_{A}\right)=\phi\left(\frac{1}{2} \operatorname{Tr}_{4}+\frac{1}{2} R_{4}\right) \leqslant \frac{1}{2},
$$

this means that we must have equality throughout. In particular, equality in the last inequality yields

$$
\phi\left(W_{4}+L_{4}\right)=0
$$

which means that $\phi$ is locally transitive.

For more complex applications of the semidefinite method, see $\left[\mathrm{BHL}^{+} 15, \mathrm{CKP}^{+} 13\right.$, $\mathrm{DHM}^{+}$13, FRV13, PV13, Raz10].

\section{B.2 Differential method}

Let us start with the definition of a vertex uniform theory.

Definition B.1 ([Raz07, Definition 11]). A theory $T$ is vertex uniform if it has only one model of size 1 . In this case, we denote the unique type of size 1 by 1 .

Remark B.2. Most of the theories considered so far $\left(T_{\text {Graph }}, T_{\text {Digraph }}, T_{\text {Tournaments }}, T_{\text {Perm }}\right.$, etc.) are vertex uniform. An example of a theory that is not vertex uniform is the theory of graphs where we allow loops, that is, we remove axiom (i) from $T_{\text {Graph }}$ (see Example 1.1.1).

The idea of the differential method is that when the target function of an extremal problem is smooth enough, we can use analysis arguments to reason about locally extremal homomorphisms. Let us define some notation to make this precise.

Definition B.3. Let $\vec{M}=\left(M_{1}, M_{2}, \ldots, M_{h}\right)$ be a sequence (vector) of models, let $\phi \in$ $\operatorname{Hom}^{+}\left(\mathcal{A}^{0}, \mathbb{R}\right)$ be a homomorphism.

We denote by $\phi(\vec{M})$ the vector $\left(\phi\left(M_{1}\right), \phi\left(M_{2}\right), \ldots, \phi\left(M_{h}\right)\right) \in \mathbb{R}^{h}$. 
Let $f: U \rightarrow \mathbb{R}$ be a function on an open neighbourhood $U$ of $\phi(\vec{M})$. We say that $\phi$ is locally extremal for $f$ and $\vec{M}$ if there exists an open neighbourhood $V \subset U$ of $\phi(\vec{M})$ such that for every homomorphism $\psi \in \operatorname{Hom}^{+}\left(\mathcal{A}^{0}, \mathbb{R}\right)$ with $\psi(\vec{M}) \in V$, we have

$$
f(\psi(\vec{M})) \geqslant f(\phi(\vec{M})) .
$$

(By further restricting the domain of $f$, we may suppose $U=V$.)

Suppose furthermore that $f$ is continuously differentiable in $U$ and that $a \in U$. The model gradient of $f$ relative to $\vec{M}$ and $a$, denoted by $\operatorname{Grad}_{\vec{M}, a}(f)$ is the formal dot product of the (calculus) gradient $\nabla f(a)$ of $f$ at a with the vector $\vec{M}$, that is, we have

$$
\operatorname{Grad}_{\vec{M}, a}(f)=\nabla f(a) \cdot \vec{M}=\sum_{i=1}^{h} \frac{\partial f}{\partial x_{i}}(a) \cdot M_{i} \quad \in \mathcal{A}^{0} .
$$

Let us try to use some standard analysis argument to find restrictions on a locally extremal homomorphism $\phi$. First, note that for these purposes, we can think of $\phi$ as the point $\phi(\vec{M})$ in $U \subset \mathbb{R}^{h}$. The standard analysis argument says that at any local minimum $t$ of this set, we must have $\nabla f(t)=0$.

However since the set

$$
\left\{\psi(\vec{M}): \psi \in \operatorname{Hom}^{+}\left(\mathcal{A}^{0}, \mathbb{R}\right)\right\} \cap U
$$

may be (and actually is) a proper subset of $U$, we must consider how does this restricts the set $U$.

One first approach would be to use Lagrange multipliers, since the restrictions that make $\psi$ a positive homomorphism are smooth (they all come from equalities involving sums and multiplications or from semi-space restrictions). Unfortunately, there are countably infinite many restrictions which makes this approach very little attractive.

Another approach is to note that, since the restrictions are all smooth, we know that if $f$ has a local minimum at $t$, then

$$
\nabla f(t) \cdot \vec{v}=0
$$

for every "valid" direction $\vec{v}$, where by "valid" direction we mean a direction that respects the Lagrange multipliers. Fortunately, some "valid" directions can be found without even mentioning Lagrange multipliers, for instance, if we start with a locally extremal homomorphism $\phi$ and delete a vertex from $\phi$, thinking of $\phi$ as a large model, then we are moving in a "valid" direction since the result would still be a large model. This is exactly the intuition of the vertex deletion operator presented below.

Lemma B.4 ([Raz07, Lemma 4.2ab]). Suppose $T$ is a vertex uniform universal theory. For every model $M \in \mathcal{M}_{\ell}$ of size $\ell$, let

$$
\partial_{1}(M)=\ell\left(\pi^{1}(M)-\mu^{1}(M)\right)
$$

where

$$
\mu^{1}(M)=\sum_{\substack{F \in \mathcal{F}^{1}: \\\left.F\right|_{0} \cong M}} F
$$


and extend $\partial_{1}$ linearly to $\mathbb{R} \mathcal{M}$.

Under these circumstances, the following hold.

a) If $M$ and $N$ are models of sizes $\ell$ and $L$ respectively with $L \geqslant \ell+1$ and $v \in V(N)$ is a vertex of $N$, then

$$
p(M ; N-v)-p(M ; N)=\frac{p\left(\partial_{1}(M) ;(N, v)\right)}{L},
$$

where $N-v$ denotes the model $\left.N\right|_{V(N) \backslash\{v\}}$ and $(N, v)$ denotes the 1-flag obtained from $N$ by labelling $v$.

b) $\partial_{1}\left(\mathcal{K}^{0}\right) \subset \mathcal{K}^{1}$ and, therefore, this operator induces a linear operator from $\mathcal{A}^{0}$ to $\mathcal{A}^{1}$, called vertex deletion differential operator.

The next theorem captures the intuition that vertex deletion is a "valid" direction.

Theorem B.5 ([Raz07, Theorem 4.3]). If $\phi \in \operatorname{Hom}^{+}\left(\mathcal{A}^{0}, \mathbb{R}\right)$ is a locally extremal homomorphism for the continuously differentiable function $f: U \rightarrow \mathbb{R}^{h}$ and the vector $\vec{M}=$ $\left(M_{1}, \ldots, M_{h}\right)$ of models of a vertex uniform theory $T$, then

$$
\phi^{\mathbf{1}}\left(\partial_{1}\left(\operatorname{Grad}_{\vec{M}, \phi(\vec{M})}(f)\right)\right)=0 \text { a.s. }
$$

In particular, the following corollary allows us to get information on locally extremal homomorphisms in the 0-flag algebra.

Corollary B.6 ([Raz07, Corollary 4.6a]). Under the same assumptions as Theorem B.5, if $g \in \mathcal{A}^{1}$ is a 1-flag algebra element, then

$$
\phi\left(\llbracket \partial_{1}\left(\operatorname{Grad}_{\vec{M}, \phi(\vec{M})}(f)\right) g \rrbracket_{1}\right)=0 .
$$

As a final remark of this section, let us mention that there are also other differential operators with properties similar to $\partial_{1}$, for instance, see [Raz07, Lemma 4.4, Theorem 4.5 and Corollary 4.6b] for the differential operator corresponding to edge deletion in $T_{\text {Graph }}$.

For applications of the differential method, see [Raz07, Section 5] and [Raz08]. 


\section{Bibliography}

[BEHJ95] B. Bollobás, Y. Egawa, A. Harris and G. P. Jin. The maximal number of induced r-partite subgraphs. Graphs Combin., 11(1):1-19, 1995.

$\left[\mathrm{BHL}^{+} 15\right]$ J. Balogh, P. Hu, B. Lidickỳ, O. Pikhurko, B. Udvari and J. Volec. Minimum number of monotone subsequences of length 4 in permutations. Combin. Probab. Comput., 24(4):658-679, 2015.

[BR13] V. Bhat and V. Rödl. Note on upper density of quasi-random hypergraphs. Electron. J. Combin., 20(2):Paper 59, 8, 2013.

[Bro80] A. E. Brouwer. The enumeration of locally transitive tournaments, volume 138 of Afdeling Zuivere Wiskunde [Department of Pure Mathematics]. Mathematisch Centrum, Amsterdam, 1980.

[CG90] F. Chung and R. Graham. Quasi-random hypergraphs. Random Structures Algorithms, 1(1):105-124, 1990.

[CG91] F. Chung and R. Graham. Quasi-random tournaments. J. Graph Theory, 15(2):173-198, 1991.

[CGW89] F. Chung, R. Graham and R. Wilson. Quasi-random graphs. Combinatorica, 9:345-362, 1989.

[Chu12] F. Chung. Quasi-random hypergraphs revisited. Random Structures Algorithms, 40(1):39-48, 2012.

$\left[\mathrm{CKP}^{+} 13\right]$ J. Cummings, D. Král', F. Pfender, K. Sperfeld, A. Treglown and M. Young. Monochromatic triangles in three-coloured graphs. J. Combin. Theory Ser. B, 103(4):489-503, 2013.

[Coo04] J. N. Cooper. Quasirandom permutations. J. Combin. Theory Ser. A, 106(1):123$143,2004$.

[Cor15] L. N. Coregliano. Quasi-carousel tournaments. 2015. Submitted. Pre-print available at http://arxiv.org/abs/1503.04124.

[CR15] L. N. Coregliano and A. A. Razborov. On the density of transitive tournaments. 2015. Submitted. Pre-print available at http://arxiv.org/abs/1501.04074.

[DCF00] D. De Caen and Z. Füredi. The maximum size of 3-uniform hypergraphs not containing a Fano plane. J. Combin. Theory Ser. B, 78(2):274-276, 2000.

$\left[\mathrm{DHM}^{+} 13\right]$ S. Das, H. Huang, J. Ma, H. Naves and B. Sudakov. A problem of Erdös on the minimum number of $k$-cliques. J. Combin. Theory Ser. B, 103(3):344-373, 2013. 
[DJ08] P. Diaconis and S. Janson. Graph limits and exchangeable random graphs. Rend. Mat. Appl. (7), 28(1):33-61, 2008.

[Erd62] P. Erdős. On the number of complete subgraphs contained in certain graphs. Publ. Math. Inst. Hungar. Acad. Sci, 7:459-464, 1962.

[ES12] G. Elek and B. Szegedy. A measure-theoretic approach to the theory of dense hypergraphs. Adv. Math., 231(3-4):1731-1772, 2012.

[FRV13] V. Falgas-Ravry and E. R. Vaughan. Applications of the semi-definite method to the Turán density problem for 3-graphs. Combin. Probab. Comput., 22(1):21-54, 2013.

[Goo59] A. W. Goodman. On sets of acquaintances and strangers at any party. Amer. Math. Monthly, 66:778-783, 1959.

[Gri13] S. Griffiths. Quasi-random oriented graphs. J. Graph Theory, 74(2):198-209, 2013.

[HHN14] H. Hatami, J. Hirst and S. Norine. The inducibility of blow-up graphs. J. Combin. Theory Ser. B, 109:196-212, 2014.

[HKM $\left.{ }^{+} 13\right]$ C. Hoppen, Y. Kohayakawa, C. G. Moreira, B. Ráth and R. M. Sampaio. Limits of permutation sequences. J. Combin. Theory Ser. B, 103(1):93-113, 2013.

[HN11] H. Hatami and S. Norine. Undecidability of linear inequalities in graph homomorphism densities. J. Amer. Math. Soc., 24(2):547-565, 2011.

[KP13] D. Král' and O. Pikhurko. Quasirandom permutations are characterized by 4-point densities. Geom. Funct. Anal., 23(2):570-579, 2013.

[KS06] M. Krivelevich and B. Sudakov. Pseudo-random graphs. In More Sets, Graphs and Numbers, Bolyai Society Mathematical Studies 15, pages 199-262. Springer-Verlag, 2006.

[KS13] S. Kalyanasundaram and A. Shapira. A note on even cycles and quasirandom tournaments. J. Graph Theory, 73(3):260-266, 2013.

[LS06] L. Lovász and B. Szegedy. Limits of dense graph sequences. J. Combin. Theory Ser. B, 96(6):933-957, 2006.

[Mye03] J. S. Myers. The minimum number of monotone subsequences. Electron. J. Combin., 9(2):Research paper 4, 17 pp. (electronic), 2002/03. Permutation patterns (Otago, 2003).

[PV13] O. Pikhurko and E. R. Vaughan. Minimum number of $k$-cliques in graphs with bounded independence number. Combin. Probab. Comput., 22(6):910-934, 2013.

[Raz07] A. A. Razborov. Flag algebras. J. Symbolic Logic, 72(4):1239-1282, 2007.

[Raz08] A. A. Razborov. On the minimal density of triangles in graphs. Combin. Probab. Comput., 17(4):603-618, 2008.

[Raz10] A. A. Razborov. On 3-hypergraphs with forbidden 4-vertex configurations. SIAM J. Discrete Math., 24(3):946-963, 2010. 
[Raz13a] A. A. Razborov. Flag algebras: an interim report, 2013.

[Raz13b] A. A. Razborov. What is. . a flag algebra? Notices Amer. Math. Soc., 60(10):13241327, 2013.

[Tho87] A. Thomason. Pseudo-random graphs. Ann. of Discrete Math., 33:307-331, 1987.

[Tho89] A. Thomason. A disproof of a conjecture of Erdős in Ramsey theory. J. London Math. Soc. (2), 39(2):246-255, 1989. 


\section{Index}

\section{2-common, 51}

a.s. (almost surely), 3, 20, 21, 23, 26, 27, 30-33, 55, 63, 64, 66

Algebra homomorphism, 19, 20, 24

Anti-parallel arc interpretation $\left(I_{\mathrm{AP}}\right), 24$

Automorphism, 9, 58

Axiom (logic), 5-9, 14, 57, 59, 64

Balanced, 31, 41-45, 48, 49, 54, 55

property, 31

tournament, 42, 45, 48

Blow-up of the graph $G$ relative to vector $\vec{k}\left(G^{(\vec{k})}\right), 53$

Boundary of the set $A(\delta(A)), 18$

Carousel

homomorphism $\left(\phi_{\mathrm{R}}\right), 41-46,48,53$

tournament of order $2 n+1\left(R_{2 n+1}\right), 4,41,42,45-49,53$

Closed formula, 57

Common

inneighbourhood, 26, 36

neighbourhood, 23

non-neighbourhood, 23

outneighbourhood, 26, 29

Complement of the graph $G(\bar{G}), 10,11,16,17,22,23,51$

Complete graph on $n$ vertices $\left(K_{n}\right), 10,11,15-17,22,23,51,53,58$

Conjunction (logic), 58

Constant symbol, 5, 6, 9, 19, 22, 24, 57-59, 61

Convergence in distribution, 18

Convergent sequence of flags, 1, 13-15, 26

Decreasing factorial $\left((n)_{k}=n(n-1) \cdots(n-k+1)\right), 17$

Degenerate type, 14, 15

Density, 1-9, 13, 21-23, 25, 26, 28, 29, 33, 35, 36, 41-43, 45, 51-54

Differential method, 4, 61, 64, 66

Distribution, 18, 21, 42, 46-48

Downward operator relative to $\sigma$ and $\eta\left(\llbracket \cdot \rrbracket_{\sigma, \eta}\right), 16-18,20,21,23,28-31,35-37,62-64,66$

Erdős-Rényi random graph of size $n$ and edge density $p\left(\boldsymbol{G}_{\boldsymbol{n}, \boldsymbol{p}}\right), 1,51$

Expected value $(\mathbb{E}), 18,23,25,26,28-33,36-38,42,43,45,48,55,64$

Extension of the theory $T$ by the type $\sigma\left(T^{\sigma}\right), 9,14,19,24$

Family 
of all (finite) $\sigma$-flags up to isomorphism $\left(\mathcal{F}^{\sigma}\right), 9,10,13-20,34,46,48,49,61-63,65$

of all (finite) $\sigma$-flags up to isomorphism that are $U$-models $\left(\mathcal{F}^{\sigma, U}\right), 19$

of all finite models up to isomorphism $(\mathcal{M}), 66$

of all models of size $k$ up to isomorphism $\left(\mathcal{M}_{k}\right), 6,9,10,51,65$

of all permutations over the set $X(\mathfrak{S}[X]), 7$

of all permutations over $[k]\left(\mathfrak{S}_{k}\right), 7,8,10,52$

of all permutations over $[k]$ for some $k \in \mathbb{N}(\mathfrak{S}), 7$

of all $\sigma$-flags of size $\ell$ up to isomorphism $\left(\mathcal{F}_{\ell}^{\sigma}\right), 9,13-15,17,19,22,26,29,36-39,43,46$, $47,62,63$

of all $\sigma$-flags up to isomorphism that are $U$-models and have size $\ell\left(\mathcal{F}_{\ell}^{\sigma, U}\right), 19,22$

Field of real numbers $(\mathbb{R}), 14-18,20-23,25,26,28-37,42,43,45,48,51,52,54,55,61-66$

First-order

formulation, 5

language, $5-7,57,58,61$

logic, 4,5

Flag, 3, 4, 9, 10, 13-22, 26, 28, 30, 32, 35, 43, 46, 47, 62, 63, 66

$\alpha, 28,31,34-38,55,63,64$

$\beta, 28,34-36,38,55,63,64$

algebra of type $\sigma\left(\mathcal{A}^{\sigma}\right), 14-26,28-37,42,43,45-48,51,52,54,55,61-66$

algebra unity of type $\sigma\left(1_{\sigma}=(\sigma\right.$ Id $\left.)\right), 9,14-18,20-23,27-33,37,38,62-64$

$\vec{C}_{3}^{A}, 3,4,26-28,30-33,42-48,63,64$

embedding, 9

$I^{A}, 3,4,26-28,30,32,34-38,43,44,46-48$

interpretation of flag $F$ by translation $I\left(I^{\prime}(F)\right), 19,20,22$

$O^{A}, 3,4,26-30,32,34,35,37,38,43,44,46-48$

removing restriction of flag $F$ through $\eta\left(F \downarrow_{\eta}\right), 20$

restriction of flag $F$ through $\eta\left(\left.F\right|_{\eta}\right), 16,18,20,26,65$

$\operatorname{Tr}_{3}^{A}, 3,4,26-28,30,32-39,43,44,46-48,64$

$\operatorname{Tr}_{3}^{\mathrm{W}}, 28$

$\operatorname{Tr}_{4}^{A}, 30$

$\operatorname{Tr}_{k}^{\mathrm{W} 2}, 28$

Formula, 19, 22, 24, 57, 58

Free variable (logic), 57-59

Function symbol, 5, 6, 19, 57, 58, 61

Global interpretation, 24

Gradient of function $f(\nabla f), 65$

Graph of inversions of permutation $\tau\left(G_{\tau}\right), 8,24$

Group of automorphisms of a model $M(\operatorname{Aut}(M)), 25,26,35-37,51$

Homomorphism, 15-18, 21-23, 26, 30-33, 36, 37, 41-43, 45, 48, 49, 51-55, 61-66

extension of $\phi$ to type $\sigma$ through $\eta\left(\phi^{\sigma, \eta}\right), 15-18,20,21,23,27-33,36-38,42-46,48,55$,

$63,64,66$

Identity

function (Id), 9, 14

permutation of size $k\left(\mathrm{Id}_{k}\right), 52$

Image (im), 9, 10, 16, 17

Increasing sequence of flags, 13, 52

Induced 
subdigraph, 6

subgraph, 6, 8

Inductive method, 4, 23

Inneighbourhood, 35, 41

Interpretation homomorphism

relative to the family of flags $\mathcal{F} \subset \mathcal{F}_{\left|\sigma_{2}\right|+1}^{\sigma_{2}}$ through $\eta\left(\pi^{\mathcal{F}, \eta}\right), 22,23$

relative to the flag $F$ through $\eta\left(\pi^{F, \eta}\right), 22,23,28,29,35-37$

relative to the open interpretation $(U, I)\left(\pi^{(U, I)}\right), 19,20,22-24,62$

Inversion interpretation $\left(I_{\text {Inv }}\right), 24$

Isomorphic $(\cong), 9,10,15,19,20,22,24,58,65$

Isomorphism, 6, 9, 14, 16, 58

Joint density $(p), 1-3,6,8-10,13-15,17,25,26,41,46,47,62,63,66$

Kernel of the type $\sigma\left(\mathcal{K}^{\sigma}\right), 14,16,20,34,66$

Language (logic), 5, 6, 8, 9, 19, 23, 58

Limit, 2, 14, 15, 26, 27, 45, 48, 49, 53

Link interpretation $\left(I_{\text {link }}\right), 24$

Literal (logic), 58

Local interpretation, 24

Localization of flag algebra $\mathcal{A}^{\sigma}$ with respect to the multiplicative system $\left\{u^{\ell}: \ell \in \mathbb{N}\right\}\left(\mathcal{A}_{u}^{\sigma}\right)$, 19,20

Locally extremal, 65, 66

Locally transitive, 41-43, 45, 48, 64

Logical connective, 58, 59

Model, 5-10, 14, 15, 45, 52, 57-59, 61, 64-66

embedding, 9, 16, 17, 58

gradient of function $f$ relative to $\vec{M}$ and point $a\left(\operatorname{Grad}_{\vec{M}, a}(f)\right), 65,66$

induced, 6, 57

interpretation of model $M$ by translation $I(I(M)), 19,24,59$

restriction of the model $M$ to the set $W\left(\left.M\right|_{W}\right), 6,7,9,14,19,20,57,66$

Multiplicative system, 19

Neighbourhood, 23

Non-degenerate type, 14-16, 18-21, 24, 61

Non-neighbourhood, 23

Normalizing factor of $\sigma$-flag $F$ through $\eta\left(q_{\sigma, \eta}(F)\right), 16-18$

Occurrence, 6-8, 15, 21, 22, 45, 46, 54

Open

diagram, 7, 9, 14, 58

formula, 24, 57-59

interpretation $(U, I)$ of $T_{1}$ in $T_{2}\left((U, I): T_{1} \rightsquigarrow T_{2}\right), 19,24,59$

Operation $\tau^{F}, 34-39$

Operator $\mu^{1}, 65$

Order induced by the permutation $\tau\left(<_{\tau}\right), 7,8$

Ordinary cone relative to $\mathcal{F}^{\sigma}\left(\mathcal{C}\left(\mathcal{F}^{\sigma}\right)\right), 61-63$

Orientation-erasing interpretation $\left(I_{\mathrm{OE}}\right), 24$

Outneighbourhood, 35, 41, 42 
Path on $n$ vertices $\left(P_{n}\right), 10,11,16,17,22,23$

Permutation, 7, 8

Predicate symbol, 5, 6, 8, 20, 24, 57-59

Preorder relative to type $\sigma\left(\leqslant_{\sigma}\right), 61-64$

Probability $(\mathbb{P}), 6,9,15,16,21,22,25,26,46$

Probability measure extending $F$ to $\sigma$ through $\eta\left(\mathbb{P}_{F}^{\eta}\right), 17,18,46,47$

Quantifier (logic), 57

Quasi-

carousel property, 3, 4, 41-45

carouselness, 4, 43

random, $2,3,25,51,52,54,55$

homomorphism $\left(\phi_{\mathrm{qr}}\right), 25,26,28-34,36-38,41,43,44,52,55$

homomorphism in $T_{\text {Graph }}\left(\phi_{\text {Graph,qr }}\right), 51$

homomorphism in $T_{\text {Perm }}\left(\phi_{\text {Perm,qr }}\right), 51,52$

property, 3, 27, 28, 30, 31, 41, 43, 44, 55

randomness, $1,4,25,26,32,51$

Random tournament of size $n\left(\boldsymbol{R}_{\boldsymbol{n}, \mathbf{1 / 2}}\right), 2,25,26,53$

Reverse permutation of size $k\left(\operatorname{Rev}_{k}\right), 52$

Sameness set, 23

Semantic cone relative to $\mathcal{F}^{\sigma}\left(\mathcal{C}_{\text {sem }}\left(\mathcal{F}^{\sigma}\right)\right), 61-63$

Semidefinite

method, 4, 31, 52, 61-64

program, 61, 63, 64

Set

of all types $\sigma_{2}$ such that $\left(\sigma_{2}, \eta\right)$ is a type extension of $\sigma(\operatorname{Ext}(\sigma, \eta)), 21$

of Borel sets of the topological space $X(\mathcal{B}(X)), 18$

of formal $\mathbb{R}$-linear combinations of elements of $X(\mathbb{R} X), 13,14,16,19,34,36,37,66$

of homomorphisms from $\mathcal{A}^{\sigma}$ to $\mathbb{R}\left(\operatorname{Hom}\left(\mathcal{A}^{\sigma}, \mathbb{R}\right)\right), 15$

of minimization quasi-random characterizers $\left(\mathcal{P}_{\mathrm{qr}}\right), 33,34,37,38$

of natural numbers $(\mathbb{N}=\{0,1,2, \ldots\}), 1-4,6-10,13,15,18,19,25,26,29,31,33,38$, $41,42,45,46,48,49,51-53,61,62$

of positive homomorphisms from $\mathcal{A}^{\sigma}$ to $\mathbb{R}\left(\mathrm{Hom}^{+}\left(\mathcal{A}^{\sigma}, \mathbb{R}\right)\right), 15-18,20-23,25,26,28-37$, $42,43,45,48,51,52,54,55,61-66$

of positive integers smaller or equal to $k([k]=\{1,2, \ldots, k\}), 7-9,14,16,17,19-22,25$,

$42,46,47,53,58$

of quasi-randomly minimized elements $\left(\mathcal{Q}_{\mathrm{qr}}\right), 33,34,36-38$

of vertices of the model $M(V(M)), 1-3,5-9,14,16,17,19-21,24,25,35,38,41,42,53$, $57-59,66$

Size of a flag $(|\cdot|), 1-3,6,7,9,10,13-17,19-22,24-26,28-32,35,38,43,45-49,51-53,62$, 64-66

Strict orientation-erasing interpretation $\left(I_{\mathrm{SOE}}\right), 24$

Subflag, 10, 16

Submodel, 6, 7, 14

density, 6

Subtournament, 6

Theory

of $k$-uniform hypergraphs ( $\left.T_{k \text {-hypergraph }}\right), 8,24$ 
of Digraphs $\left(T_{\text {Digraph }}\right), 6,24,64$

of Graphs $\left(T_{\text {Graph }}\right), 1,5,6,10,16,22-24,51,58,64,66$

of Graphs of Inversions ( $\left.T_{\text {Graphofinversions }}\right), 8,24$

of Permutations $\left(T_{\text {Perm }}\right), 7,8,10,16,22,24,51,52,64$

of Tournaments ( $\left.T_{\text {Tournament }}\right), 2,3,6,10,23-25,34,41,45,52-55,64$

Theory (logic), 6-10, 14-16, 19, 20, 23, 25, 41, 45, 51, 57, 59, 61, 64, 66

Tournament

3-cycle $\left(\vec{C}_{3}\right), 26-28,31-33,48,54,63$

$L_{4}, 27,31,32,41,43,45,53-55$

$R_{4}, 3,4,26,27,31-33,38,42-45,48,53,55,64$

$W_{4}, 27,31,32,41,43,45,53-55$

Transitive, 3, 29, 41

homomorphism $\left(\phi_{\operatorname{Tr}}\right), 29,32,33$

tournament of size $k\left(\operatorname{Tr}_{k}\right), 2-4,25-34,37,38,43-45,48,51,53,55,63,64$

Translation, 19, 20, 24, 58, 59

Type, 9, 10, 13-16, 18-21, 24, 26, 28, 58, 61-64

$0,9,16,18,20,22-39,42,43,45,48,49,51,52,54,55,62-66$

$1,10,11,16,17,22-24,28,31,35-37,55,63-66$

A, 3, 4, 26-33, 35-38, 42-48, 64

$E, 10,17,22,23$

$\bar{E}, 10,17,22$

extension, 16-18, 20-22, 62

induced by $\eta$ on $\sigma\left(\left.\sigma\right|_{\eta}\right), 16$

U-model, 19, 59

Unique $\sigma$-flag over $M\left(M^{\sigma}\right), 9-11,16,17,22,23$

Universal theory, 2, 5-9, 14, 19, 45, 52, 57-59, 61, 65

Upward operator relative to type extension $(\sigma, \eta)\left(\pi^{\sigma, \eta}\right), 20-23,65$

Variable, 58, 59

Variance (Var), 3, 32, 33, 43, 44

Vertex deletion differential operator $\left(\partial_{1}\right), 65,66$

Vertex uniform, 64-66

Weak convergence, 17, 18, 46

Well-formed formula, 58, 59

Zero divisor, 19 